The Astrophysical Journal, 681:594-625, 2008 July 1

(C) 2008. The American Astronomical Society. All rights reserved. Printed in U.S.A.

\title{
UV EXCESS MEASURES OF ACCRETION ONTO YOUNG VERY LOW MASS STARS AND BROWN DWARFS
}

\author{
Gregory J. Herczeg ${ }^{1}$ and Lynne A. Hillenbrand ${ }^{1}$ \\ Received 2007 November 6; accepted 2008 January 16
}

\begin{abstract}
Low-resolution spectra from 3000 to $9000 \AA$ of young low-mass stars and brown dwarfs were obtained with LRIS on Keck I. The excess UV and optical emission arising in the Balmer and Paschen continua yields mass accretion rates ranging from $2 \times 10^{-12}$ to $10^{-8} M_{\odot} \mathrm{yr}^{-1}$. These results are compared with HST STIS spectra of roughly solar-mass accretors with accretion rates that range from $2 \times 10^{-10}$ to $5 \times 10^{-8} M_{\odot} \mathrm{yr}^{-1}$. The weak photospheric emission from M dwarfs at $<4000 \AA$ leads to a higher contrast between the accretion and photospheric emission relative to higher mass counterparts. The mass accretion rates measured here are systematically $\sim 4-7$ times larger than those from $\mathrm{H} \alpha$ emission line profiles, with a difference that is consistent with but unlikely to be explained by the uncertainty in both methods. The accretion luminosity correlates well with many line luminosities, including high Balmer and many He I lines. Correlations of the accretion rate with $\mathrm{H} \alpha 10 \%$ width and line fluxes show a large amount of scatter. Our results and previous accretion rate measurements suggest that $\dot{M} \propto M^{1.87 \pm 0.26}$ for accretors in the Taurus molecular cloud.
\end{abstract}

Subject headings: planetary systems: protoplanetary disks — stars: pre-main-sequence

\section{INTRODUCTION}

In the magnetospheric accretion model for accretion onto young stars, the stellar magnetosphere truncates the disk at a few stellar radii. Gas from the disk accretes onto the star along magnetic field lines and shocks at the stellar surface. The $\sim 10^{4} \mathrm{~K}$ optically thick postshock gas and optically thin preshock gas produce emission in the Balmer and Paschen continua and in many lines, including the Balmer and Paschen series and the $\mathrm{Ca}$ II IR triplet (Hartmann et al. 1994; Calvet \& Gullbring 1998). The accretion luminosity may be estimated from any of these accretion tracers and subsequently converted into a mass accretion rate.

Historically, mass accretion rates onto young stars have been measured directly using optical veiling from the Paschen continuum and stronger Balmer continuum emission shortward of the Balmer jump. Modeling of the Paschen and Balmer continuum emission with a generic isothermal, plane-parallel slab yields $\dot{M} \sim 10^{-7}$ to $10^{-10} M_{\odot} \mathrm{yr}^{-1}$ for accretors with masses of 0.4-1.0 $M_{\odot}$ (Valenti et al. 1993; Gullbring et al. 1998). Calvet \& Gullbring (1998) obtained similar accretion rates by applying more realistic shock geometries with a range of temperatures to explain the observed accretion continuum. The accretion luminosity can be measured instead at longer wavelengths with optical veiling measurements from high-dispersion spectra (e.g., Basri \& Batalha 1990; Hartigan et al. 1991, 1995; Hartigan \& Kenyon 2003; White \& Hillenbrand 2004), albeit with a larger uncertainty in bolometric correction.

Several deep surveys have since identified large populations of young very low mass stars and brown dwarfs with masses $<0.2 M_{\odot}$ (e.g., Briceño et al. 1998; Luhman 2004; Slesnick et al. 2006; Guieu et al. 2007). Excess IR emission indicates the presence of disks around some of these very low mass objects ${ }^{2}$ (e.g., Muzerolle et al. 2003; Jayawardhana et al. 2003; Natta et al. 2004). The excess Balmer continuum emission produced by accretion onto very low mass stars and brown dwarfs has not been

\footnotetext{
1 California Institute of Technology, Pasadena, CA 91125.

${ }^{2}$ The objects studied in this paper span the mass range from 0.024 to $0.2 M_{\odot}$; hereafter all are referred to as "stars" for ease of terminology. We refer to $\sim 0.3-$ $1 M_{\odot}$ stars as "higher mass stars."
}

observed previously. Instead, accretion has been measured based on magnetospheric models of the $\mathrm{H} \alpha$ line profile (Muzerolle et al. 2000, 2003, 2005), by extending relationships between $\dot{M}$ and line luminosities established at higher masses to brown dwarf masses (Natta et al. 2004, 2006; Mohanty et al. 2005), or in a few cases from measurements of the Paschen continuum (White \& Basri 2003).

In this paper we present the first observations of the Balmer jump, and hence direct measurements of the accretion luminosity, for young very low mass stars and brown dwarfs using data obtained with LRIS on Keck I. We compare the Balmer and Paschen continuum emission from very low mass stars to that from higher mass accretors that were observed with the Hubble Space Telescope (HST). The weak $U$-band photospheric emission of $\mathrm{M}$ dwarfs leads to a high contrast between accretion and photospheric emission at $\lambda<3700 \AA$. The properties of the Balmer continuum and jump for very low mass stars and brown dwarfs are mostly similar to those of the higher mass accretors, although the sensitivity to low $\dot{M}$ improves to smaller stellar mass. The Balmer jump tends to be larger for smaller mass accretion rates, indicating lower optical depth for the emitting gas. The luminosity in many lines, including the Balmer series and $\mathrm{He} \mathrm{I}$ lines, correlates well with the accretion luminosity. Our $\dot{M}$ estimates are systematically $\sim 4-7$ times higher than those estimated from modeling the $\mathrm{H} \alpha$ line profile. We find that $\dot{M} \propto M^{1.9}$ for accretors in Taurus and discuss biases in the selection of those accretors that may affect this calculated relationship.

\section{OBSERVATIONS}

\subsection{Keck I LRIS}

We used LRIS (Oke et al. 1995; McCarthy et al. 1998) on Keck I to obtain low-resolution optical spectra covering 3000$9000 \AA$ of 17 young, low-mass Taurus sources and 2M 12073932 on 2006 November 23. We observed 2M 0436+2351 and obtained a deeper observation of 2M 1207-3932 on 2007 February 7. Our observation log (Table 1) lists the exposure time for each target and abbreviations for each target used throughout this paper. 
TABLE 1

Observations with Keck I LRIS

\begin{tabular}{|c|c|c|c|c|c|c|c|c|}
\hline Star & $\begin{array}{c}\text { MJD } \\
(540,000)\end{array}$ & $t_{\text {exp }}$ & $U^{\mathrm{a}}$ & $B^{\mathrm{a}}$ & $V^{\mathrm{a}}$ & $R^{\mathrm{a}}$ & $\begin{array}{c}\sigma^{\mathrm{b}} \\
(\mathrm{mag})\end{array}$ & Abbreviation $^{c}$ \\
\hline 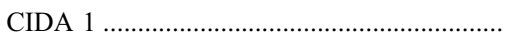 & 63.318 & 720 & 18.8 & 18.4 & 17.3 & 15.3 & 0.6 & CIDA 1 \\
\hline 2MASS J04141188+2811535 ........................ & 63.329 & 1200 & 18.3 & 18.2 & 17.6 & 16.4 & 0.6 & $2 \mathrm{M} 0414+2811$ \\
\hline MHO Tau 5 & 63.382 & 1620 & 19.6 & 18.5 & 17.0 & 15.0 & 0.4 & MHO 5 \\
\hline 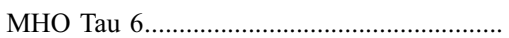 & 63.405 & 1260 & 18.7 & 17.9 & 16.6 & 15.1 & 0.4 & MHO 6 \\
\hline GM Tau.................................... & 63.422 & 1050 & 18.8 & 18.8 & 17.8 & 16.3 & 0.4 & GM Tau \\
\hline 2MASS J04390396+2544264_..................... & 63.438 & 2700 & 22.7 & 21.9 & 20.2 & 17.6 & 0.4 & $2 \mathrm{M} 0439+2544$ \\
\hline 2MASS J04442713+2512164_...................... & 63.475 & 2100 & 21.3 & 20.5 & 19.5 & 17.3 & 0.4 & $2 \mathrm{M} 0444+2512$ \\
\hline 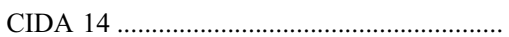 & 63.498 & 720 & 18.9 & 17.4 & 16.0 & 14.4 & 0.4 & CIDA 14 \\
\hline 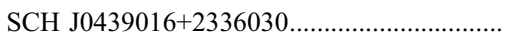 & 63.508 & 840 & 18.3 & 17.3 & 16.0 & 14.6 & 0.3 & S0439+2336 \\
\hline 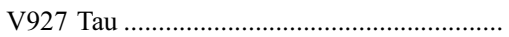 & 63.520 & 150 & 17.6 & 16.2 & 14.8 & 13.3 & 0.3 & V927 Tau \\
\hline 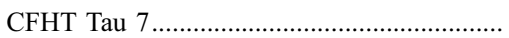 & 63.525 & 600 & 21.7 & 20.2 & 18.5 & 16.3 & 0.2 & CFHT 7 \\
\hline MHO Tau 7 & 63.532 & 600 & 19.5 & 18.1 & 16.6 & 15.0 & 0.2 & MHO 7 \\
\hline 2MASS J04381486+2611399 …................. & 63.540 & 300 & $\ldots$ & $\ldots$ & $\ldots$ & 20.1 & 0.2 & $2 \mathrm{M} 0438+2611$ \\
\hline KPNO Tau 11 & 63.552 & 900 & 20.6 & 19.3 & 17.7 & 15.9 & 0.2 & KPNO 11 \\
\hline 2MASS J04414825+2534304_..................... & 63.567 & 2400 & 22.2 & 22.0 & 21.1 & 19.1 & 0.2 & $2 \mathrm{M} 0441+2534$ \\
\hline 2MASS J04554757+3028077........................ & 63.600 & 1080 & 19.3 & 18.0 & 16.5 & 14.9 & 0.2 & $2 \mathrm{M} 0455+3028$ \\
\hline 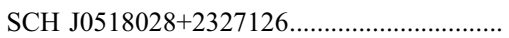 & 63.611 & 1380 & 18.3 & 18.3 & 17.8 & 16.6 & 0.2 & S $0518+2327$ \\
\hline 2MASS J12073347-3932540 ${ }^{\mathrm{d}} \ldots \ldots \ldots \ldots \ldots \ldots \ldots \ldots \ldots \ldots \ldots \ldots$ & 63.647 & 840 & 21.3 & 21.5 & 20.6 & 18.2 & 0.2 & $2 \mathrm{M} 1207-3932$ \\
\hline 2MASS J04362151+2351165 ....................... & 139.218 & 400 & 20.1 & 19.6 & 18.4 & 16.9 & 1.0 & $2 \mathrm{M} 0436+2351$ \\
\hline 2MASS J12073347-3932540 ..................... & 139.611 & 1800 & 20.9 & 21.0 & 20.3 & 18.0 & 0.2 & 2M 1207-3932 \\
\hline
\end{tabular}

a Synthetic photometry, calculated by convolving the spectra with Bessell UBVR filter transmission curves for the Nordic Optical Telescope at http://www not.iac.es/instruments / filters/.

b Approximate flux uncertainty in magnitudes.

c Abbreviation for target used throughout this work.

${ }^{\mathrm{d}}$ Used a $0.7^{\prime \prime}$ slit width.

Most targets were observed with a $175^{\prime \prime} \times 1.0^{\prime \prime}$ slit. The light was split into separate red and blue beams with the D560 dichroic. The red light illuminates a $2048 \times 2048$ CCD with $0.21^{\prime \prime}$ pixels, and the blue light illuminates a $2048 \times 4096 \mathrm{CCD}$ with $0.135^{\prime \prime}$ pixels. We used the $400 / 8500$ grism and the GG495 filter in the red channel and the 400/3400 grism in the blue channel. Each red pixel covers $\sim 1.85 \AA$, yielding a resolution $R \sim 1400$ over the $5400-9000 \AA$ wavelength range. Each blue pixel covers $\sim 1.1 \AA$, yielding a resolution $R \sim 700$ over the $3000-5700 \AA$ wavelength range. For each target we typically obtained multiple red spectra during one to two long blue exposures.

We bias-subtracted the 2006 November 23 blue exposures and estimated a bias subtraction for the 2007 February 7 blue exposure from images with no blue emission. An overscan subtraction was applied to all red images. The red images were flat-fielded with dome flats, the blue images from 2006 November were flatfielded with sky flats, and the blue images from 2007 February were flat-fielded with halogen lamp flats. We located each spectrum on the detector as a function of position in the dispersion direction. The counts in the red and blue spectra were extracted with 11 and 13 pixel widths, respectively. The sky background was measured from nearby windows and subtracted from the spectrum. The spectral regions within $25 \AA$ of 4590 and $5270 \AA$ include a transient ghost and are ignored.

The relative wavelength solution was calculated from an $\mathrm{HgNeArCdZn} \mathrm{lamp} \mathrm{spectrum.} \mathrm{The} \mathrm{absolute} \mathrm{wavelength} \mathrm{solution}$ for each observation was calculated by measuring the positions of sky emission lines.

All stars were observed at the parallactic angle. On November 23 the seeing improved from $0.9^{\prime \prime}$ to $0.7^{\prime \prime}$ from the beginning to the middle of the night and remained stable at $0.7^{\prime \prime}$ for the second half of the night. On February 7 the seeing was $0.7^{\prime \prime}$ during our observation of $2 \mathrm{M} 0436+2351$ and $1.2^{\prime \prime}$ during our observation of 2M 1207-3932.
We obtained observations of the spectrophotometric standards (Massey et al. 1988; Oke 1990) PG 0205+134 and PG 0939+262 at the beginning and end of the 2006 November run and G191B2B and PG $1545+035$ on 2007 February 7 with the $1.0^{\prime \prime}$ aperture. The November 23 standards have published fluxes from 3100 to $8000 \AA$. We approximate the flux of PG $0939+262$ from 8000 to $9000 \AA$ by assuming that the white dwarf spectrum is a blackbody, and we confirm this calibration by comparing the spectra of 2M 1207-3932 obtained on both nights. The atmospheric extinction correction was obtained from multiple observations of G191-B2B on 2007 February 7.

Slit losses were $\sim 70 \%$ at the beginning of the night and $\sim 22 \%$ at the end of the night in 2006 November. In adjacent red spectra of the same target, the extinction-corrected count rate increased early in the night and remained stable during the second half of the night, mirroring similar changes in the seeing. This increase in count rate can account entirely for the factor of $\sim 3$ in slit losses. The count rates in the first half of the night are therefore multiplied by a factor of $1-3$, based on when the observation occurred. The small wavelength dependence in this factor is found by interpolating from the observations of the photometric standards.

Table 1 lists synthetic $U B V R$ magnitudes for each source and our estimated uncertainty in absolute flux calibration. The estimated photometry is within $0.1-0.5$ mag of most existing literature measurements, with the exception of several strong accretors. The initial flux calibration of 2M 1207-3932 on the two nights differed by $0.27 \mathrm{mag}$. We estimate that the absolute fluxes are calibrated to at worst a factor of 2 early in the night and a factor of 1.3 in the second half of the night for the 2006 November observations.

\subsection{Archival HST Spectra}

We supplement our new Keck data with existing blue HST Space Telescope Imaging Spectrograph (STIS) spectra of late K 
TABLE 2

Archival HST STIS G430L Observations

\begin{tabular}{|c|c|c|c|c|c|c|}
\hline Star & MJD & $\begin{array}{c}t_{\exp } \\
\text { (s) }\end{array}$ & Slit & PI & References & Abbreviation \\
\hline DF Tau ......................... & $51,439.84$ & 144 & $52 \times 0.2$ & Linsky & 1 & DF Tau (1999 Sep) \\
\hline TW Hya ...................... & $51,671.88$ & 260 & $52 \times 0.2$ & Linsky & 2 & TW Hya (2000 May) \\
\hline TW Hya .................... & $52,474.58$ & 120 & $52 \times 0.2$ & Johns-Krull & & TW Hya (2002 Jul) \\
\hline 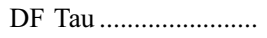 & $52,512.49$ & 120 & $52 \times 0.2$ & Johns-Krull & & DF Tau (2002 Aug) \\
\hline RU Lupi ...................... & $51,737.65$ & 120 & $52 \times 0.1$ & Walter & 1 & $\ldots$ \\
\hline V819 $\mathrm{Tau}^{\mathrm{a}} \ldots \ldots \ldots \ldots \ldots . . . . . . . .$. & $51,786.87$ & 360 & $52 \times 0.1$ & Walter & 1 & $\ldots$ \\
\hline DG Tau ...................... & $51,839.52$ & 240 & $52 \times 0.1$ & Walter & 1 & $\ldots$ \\
\hline V836 Tau ........................ & $51,954.36$ & 360 & $52 \times 0.1$ & Walter & 1 & $\ldots$ \\
\hline $\mathrm{LkCa} 7 \mathrm{~A}^{\mathrm{a}} \ldots \ldots \ldots \ldots \ldots \ldots$ & $53,039.71$ & 162 & $52 \times 0.2$ & Johns-Krull & & $\mathrm{LkCa} 7$ \\
\hline
\end{tabular}

${ }^{a}$ We extract and use only the primary spectrum and do not discuss the faint secondary.

RefERENCES.-(1) Herczeg et al. 2006; (2) Herczeg et al. 2004.

or early $\mathrm{M}$ accretors and two young nonaccretors taken from the MAST archive (see Table 2). The spectra were obtained with the G430L grating and span 2900-5700 Å with a spectral resolution of $\sim 600$. Several of these observations were discussed in Herczeg et al. (2004, 2005, 2006), including calculations of the mass accretion rate. We reanalyze these data here to provide a small set of higher mass accretors with spectra analyzed in a manner consistent with our Keck I LRIS spectra to compare with results from our sample of low-mass accretors.

\subsection{Description of Our Sample}

Several targets (GM Tau, CIDA 1, 2M 0414+2811) in our Keck I LRIS program were selected based on expectation of a high mass accretion rate inferred from strong excess continuum emission and optical line emission. Other targets were selected based on a combination of spectral type, low extinction, existing $\dot{M}$ measurements from $\mathrm{H} \alpha$, and visual magnitude. MHO 5 and KPNO 11 were selected also for a full width of the $\mathrm{H} \alpha$ line at $10 \%$ the peak flux (hereafter $\mathrm{H} \alpha 10 \%$ width) of $150-200 \mathrm{~km} \mathrm{~s}^{-1}$ (Muzerolle et al. 2003; Mohanty et al. 2005), which is intermediate between definite accretors and definite nonaccretors for their respective spectral types. The three relatively bright young stars V927 Tau, MHO 7, and CFHT 7 were chosen as photospheric templates based on spectral type and lack of strong accretion, as identified by weak $\mathrm{H} \alpha$ emission and absence of IR excess emission. Since the template stars are young, their surface gravity will be similar to the rest of the sample and provide a better match to the photospheric spectrum than could be obtained from older field dwarfs.

To obtain a larger range in $\dot{M}$ for analyzing the properties of the accretion continuum and relationships between line and continuum emission (see $\S \S 5$ and 6 ), we supplemented the Keck I LRIS spectra with HST STIS observations of stars with higher central masses. This sample consists of most available HST observations of the 3000-5000 $\AA$ region for accreting young stars with spectral types late $\mathrm{K}$ or early $\mathrm{M}$.

Table 3 describes the properties of these targets (see also Appendices A, B, C, and D). Multiple LRIS observations of 2M 1207-3932 and HST observations of DF Tau and TW Hya are treated independently throughout this paper.

\section{OBSERVATIONS OF ACCRETION DIAGNOSTICS}

Figure 1 compares three M5-M5.5 stars in our Keck LRIS spectra from 3200 to $5600 \AA$ A. Strong $\mathrm{TiO}$ and $\mathrm{CaH}$ molecular bands (Kirkpatrick et al. 1993) dominate the photospheric emission of mid-M dwarfs. At $>3700 \AA$, such features are strong from V927 Tau and S0439+2336 but shallow from S0518+2327. This veiling of the photospheric emission from S0518+2327 is attributed to excess Paschen continuum emission. S0439+2336 and S0518+2327 both exhibit strong excess emission shortward of jump at $3700 \AA$, which is attributed to excess Balmer continuum emission. Line emission, including in the Balmer series, is much stronger from S0439+2336 and S0518+2327 than from V927 Tau. The excess Balmer and Paschen continuum and emission lines detected from young stars are commonly attributed to accretion. In the following subsections we describe and measure these accretion diagnostics for each source.

\subsection{Measuring the Paschen Continuum}

Veiling $^{3}$ is measured by comparing the depth of absorption features, which are filled in by emission from the accretion continuum, to photospheric templates. These features include the $\mathrm{TiO}$ and $\mathrm{CaH}$ absorption bands and also the strong $\mathrm{Ca}$ I $\lambda 4226$ absorption line. The $\mathrm{Ca}$ I line depth may be related to chromospheric structure and may be affected by stellar rotation (Mauas \& Falchi 1996; Short et al. 1997).

The young M dwarfs V927 Tau (M5), MHO 7 (M5.5), CFHT 7 (M6.5), and 2M 0439+2544 (M7.25) serve as photospheric templates (see Appendix A). These stars provide a better match to the surface gravity and chromospheric emission of young accretors than would older field M dwarfs. 2M 0439+2544 is not an ideal template because of ongoing accretion, and V927 Tau may also be accreting (see $\S \S 3.4$ and D2). Use of $2 \mathrm{M} 0439+2544$ and V927 Tau as templates may cause us to underestimate the veiling by 0.05 and 0.02 , respectively, at $5000 \AA$ and by 0.01 each at $7000 \AA$. At $<4500 \AA$ we use CFHT 7 instead of 2 M $0439+2544$ as a photospheric template for late $\mathrm{M}$ stars.

Large veilings $(\gtrsim 2)$ are difficult to measure because the high ratio of accretion to photospheric flux masks any photospheric absorption features. However, a large error for a high value of veiling leads to only a small error in the measurement of the underlying continuum flux. Our lower detection limit of veiling measurements is $\sim 0.05-0.1$, depending on how well the photospheric template matches the spectrum. The veiling is calculated from regions that do not include obvious strong emission lines. For weaker accretors, any such lines may fill in some of the absorption and thereby artificially increase our veiling estimate for some stars, particularly at $4227 \AA$ (see Fig. 2).

Figures 2-4 show three spectral regions where veiling is measured. Table 4 lists veiling measurements and the corresponding

\footnotetext{
3 The veiling at wavelength $\lambda, r_{\lambda}$, is defined by the accretion continuum flux ( $F_{\text {observed }}-F_{\text {photosphere }}$ ) divided by the photospheric flux.
} 
TABLE 3

Adopted Stellar Properties

\begin{tabular}{|c|c|c|c|c|c|c|c|c|}
\hline Star & Spectral Type & $\log (\text { age } / y r)^{a}$ & $\begin{array}{c}A_{V} \\
(\mathrm{mag})\end{array}$ & $\begin{array}{c}T \\
(\mathrm{~K})\end{array}$ & $\begin{array}{c}L \\
\left(L_{\odot}\right)\end{array}$ & $\begin{array}{c}R \\
\left(R_{\odot}\right)\end{array}$ & $\begin{array}{c}M \\
\left(M_{\odot}\right)\end{array}$ & References \\
\hline 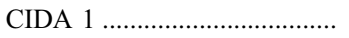 & M5 & $6.0^{*}$ & 3.0 & 3125 & 0.21 & 1.57 & 0.15 & 1 \\
\hline GM Tau.................................. & M5.5 & 6.4 & 2.0 & 3091 & 0.070 & 0.93 & 0.15 & 2 \\
\hline $2 \mathrm{M} 0414+2811 \ldots \ldots \ldots \ldots \ldots \ldots \ldots$ & M6.5 & 6.5 & 1.0 & 2935 & 0.014 & 0.46 & 0.065 & 3 \\
\hline 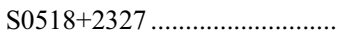 & M5.5 & 6.9 & 1.0 & 3091 & 0.016 & 0.44 & 0.10 & 4 \\
\hline 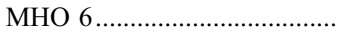 & M5 & 6.1 & 1.0 & 3125 & 0.14 & 1.31 & 0.17 & 5 \\
\hline 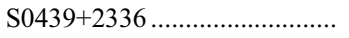 & M5 & 6.3 & 0.0 & 3125 & 0.089 & 1.02 & 0.17 & 4 \\
\hline 2M 0436+2351 ..................... & M5 & 7.1 & 1.0 & 3125 & 0.013 & 0.39 & 0.11 & 6 \\
\hline 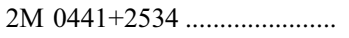 & M7.75 & 7.0 & 1.0 & 2752 & 0.0033 & 0.25 & 0.035 & 3 \\
\hline $2 \mathrm{M} 0444+2512 \ldots \ldots \ldots \ldots \ldots \ldots \ldots$ & M7.25 & 6.6 & 0.0 & 2838 & 0.012 & 0.45 & 0.050 & 3 \\
\hline 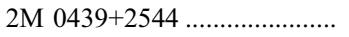 & M7.25 & 6.5 & 0.8 & 2838 & 0.015 & 0.51 & 0.050 & 3 \\
\hline 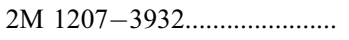 & M8 & 7.0 & 0.0 & 2550 & 0.0022 & 0.24 & 0.024 & 7 \\
\hline 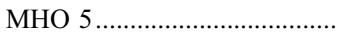 & M6 & $6.0^{*}$ & 0.3 & 2990 & 0.098 & 1.17 & 0.10 & 5 \\
\hline $2 \mathrm{M} 0455+3028 \ldots \ldots \ldots \ldots \ldots \ldots \ldots$ & M4.75 & 6.1 & 1.0 & 3161 & 0.15 & 1.31 & 0.20 & 3 \\
\hline 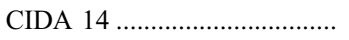 & M5.5 & 6.0 & 0.3 & 3058 & 0.16 & 1.44 & 0.12 & 1 \\
\hline 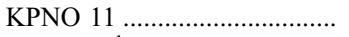 & M5.75 & 6.5 & 0.3 & 3023 & 0.037 & 0.70 & 0.10 & 8 \\
\hline 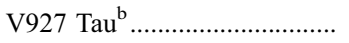 & M5 & $6.0^{*}$ & 0.4 & 3125 & 0.22 & 1.60 & 0.15 & 9 \\
\hline 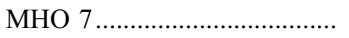 & M5.5 & 6.3 & 0.1 & 3058 & 0.076 & 0.99 & 0.14 & 5 \\
\hline 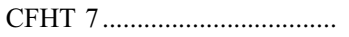 & M6.5 & 6.5 & 0.0 & 2935 & 0.025 & 0.61 & 0.07 & 10 \\
\hline \multicolumn{9}{|c|}{ HST STIS Sample } \\
\hline 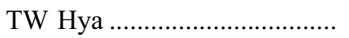 & K7 & 7.6 & 0.0 & 4060 & 0.17 & 0.83 & 0.77 & 11 \\
\hline 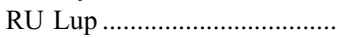 & K7 & 7.0 & 0.1 & 4060 & 0.42 & 1.3 & 1.0 & 12 \\
\hline 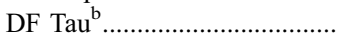 & M2 & $6.0^{*}$ & 0.6 & 3560 & 1.45 & 3.2 & 0.66 & 13 \\
\hline 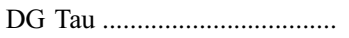 & K6 & 7.5 & 1.3 & 4205 & 0.28 & 1.0 & 0.85 & 13 \\
\hline 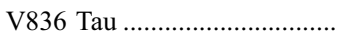 & K7 & 7.1 & 1.1 & 4060 & 0.32 & 1.15 & 0.95 & 14 \\
\hline
\end{tabular}

a 1 Myr is the youngest age of Baraffe et al. (1998) models. Ages marked with an asterisk are upper limits.

b Binary, assumes that both components have same $M, L$, and $T$.

REFERENCES.-(1) Briceño et al. 1993; (2) Rydgren \& Vrba 1983; (3) Luhman 2004; (4) Slesnick et al. 2006; (5) Briceño et al. 1998; (6) Luhman et al. 2006; (7) Gizis 2002; (8) Luhman et al. 2003; (9) Herbig \& Kameswara 1972; (10) Guieu et al. 2006; (11) Rucinski \& Krautter 1983; (12) Henize 1954; (13) Smak 1964; (14) Feigelson \& Kriss 1981.

flux in the accretion continuum at distinct wavelengths between 4000 and $9000 \AA$. Excess continuum emission is detected longward of $4000 \AA$ for many stars in our sample and is identified as the Paschen continuum. The accretion continuum is relatively flat at $<6000 \AA$ for most targets and increases at $\lambda>6000 \AA$ for

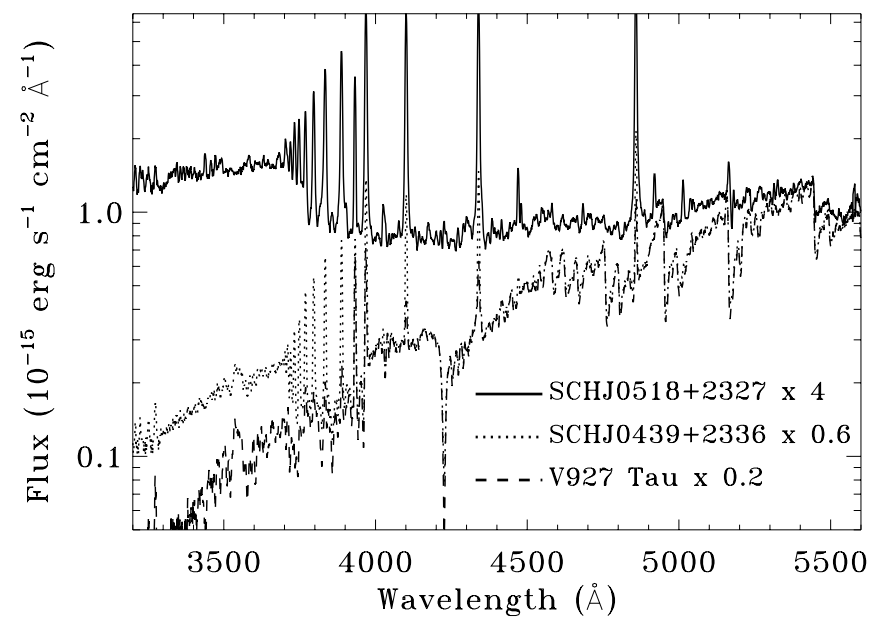

FIG. 1.- Three spectra at 3300-5600 ̊. The spectrum of V927 Tau is dominated by photospheric emission, with strong $\mathrm{TiO}$ bands and the $\mathrm{Ca}$ I $\lambda 4227 \mathrm{ab}-$ sorption line. Moderate accretors, such as S0439+2336, are dominated by photospheric emission longward of the Balmer jump at $3700 \AA$ but excess Balmer continuum emission at shorter wavelengths. The TiO bands at $<5600 \AA$ are only weakly detected from the strong accretor S0518+2327, which shows a heavily veiled spectrum both shortward and longward of the Balmer jump. most sources where it is detected. The continuum flux from $2 \mathrm{M} 0414+2811$ at $8600 \AA$ is substantially lower than that at $7800 \AA$ A, which may be consistent with weaker Brackett continuum emission longward of the Paschen jump. CIDA 1 shows no such flux reduction, which may suggest an alternate veiling source.

The early M and late K stars from the STIS data do not have the strong $\mathrm{TiO}$ and $\mathrm{CaH}$ absorption bands for veiling measurements. In several locations, the photospheric emission drops by a factor of 2-2.5 within $\sim 200 \AA$. Table 5 lists veilings at 4600 and $5400 \AA$ for this sample, measured by comparing the depth of these features with photospheric templates, and at $4000 \AA$, measured from the slope of the photospheric emission. We detect Paschen continuum emission from DF Tau, TW Hya, V836 Tau, DG Tau, and RU Lup.

\subsection{Measuring the Balmer Continuum}

When seen from young stars, excess Balmer continuum emission shortward of $\sim 3700 \AA$ is typically attributed to accretion (Fig. 5). The Balmer limit occurs at $3646 \AA$, but line blending in the Balmer series shifts the apparent jump to $3700 \AA$. The observed Balmer jump, defined here as the ratio of flux at $3600 \AA$ to the flux at $4000 \AA$, ranges from $\sim 0.35$ for the nonaccretors to 5.47 for the accretor 2M 1207-3932 (Table 6).

Figure 6 compares spectra of stars with the weakest Balmer jumps in our sample to those of photospheric templates from Table 4. Excess Balmer continuum emission is clearly detected from 2M 0455+3028, S0439+2336, and V927 Tau relative to photospheric templates. Some weak, noisy Balmer continuum 


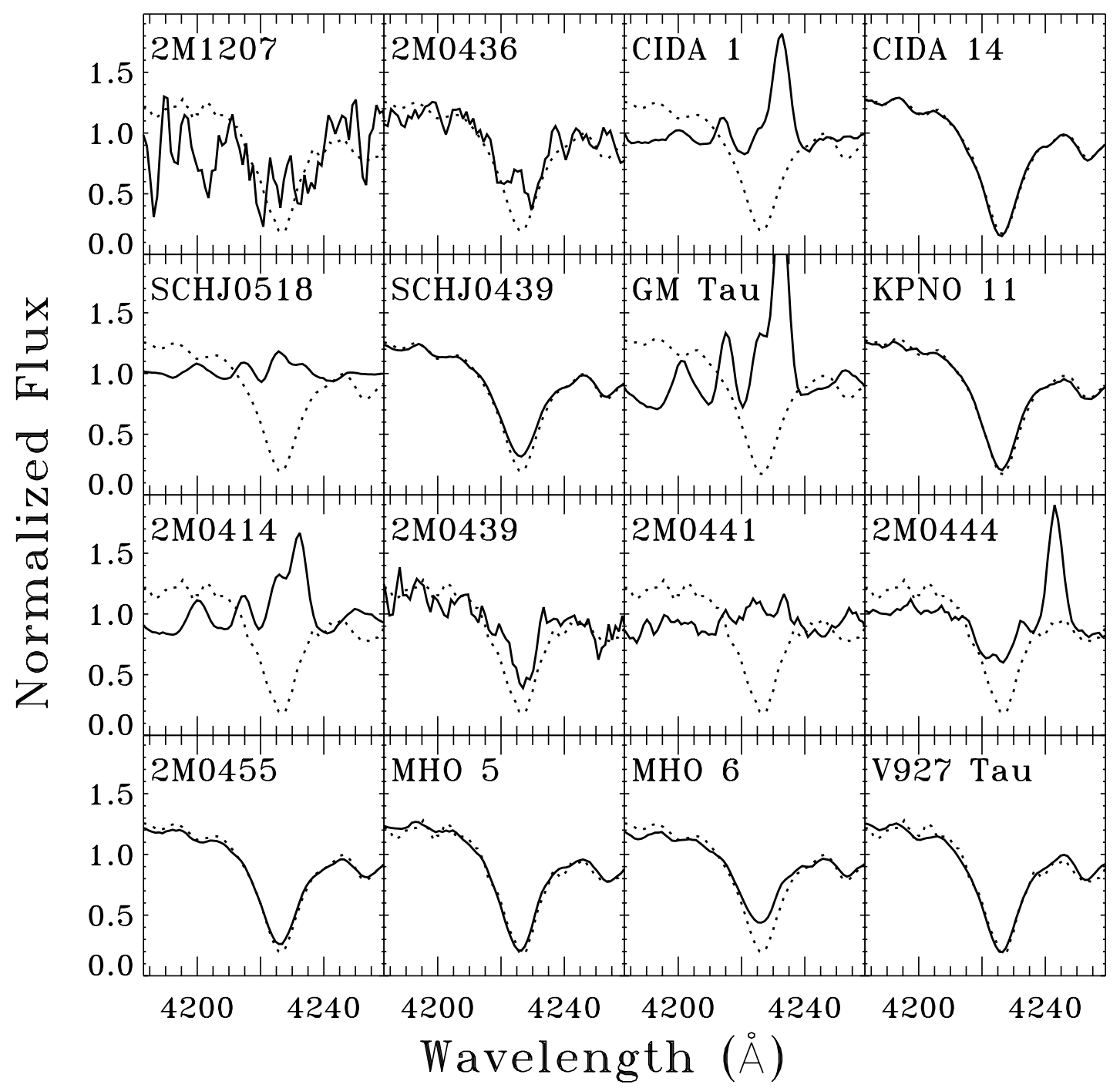

FIG. 2.-Ca I 24227 absorption line in our LRIS spectra (solid lines) compared with a photospheric template (dotted lines; template listed in Table 5). When present, accretion fills in some of the absorption. The veiling is calculated from the depth of the absorption line. Emission lines in the vicinity of the Ca I $\lambda 4227$ line from several strong accretors (e.g., CIDA 1, GM Tau) limit our ability to measure high veilings and may lead to overestimated small veilings.

emission is also detected from MHO 5 and KPNO 11. MHO 7 and CFHT 7 may each show very mild increases in emission shortward of the Balmer jump, possibly from chromospheric emission (see $\S 3.4)$.

As defined here, the Balmer jump is the ratio of Balmer plus Paschen continuum flux at $3600 \AA$ divided by the Paschen continuum flux at $4000 \AA$. The intrinsic Balmer Jump $\left(J_{\bmod }\right.$ in Table 6$)$ is measured from the accretion continuum after dereddening the spectra (see Appendix B) and subtracting a scaled photospheric spectrum. Table 6 also lists the measured slope of the continuum emission from 3200 to $3650 \AA\left(S_{B}\right)$, scaled to the observed flux at $3600 \AA$.

\subsection{Emission-Line Measurements}

Tables 7-11 list equivalent widths and fluxes for $\mathrm{H}$ Balmer lines, He lines, other strong lines associated with accretion, and lines usually associated with outflows. These tables are not complete line lists. We measure most emission-line fluxes and equivalent widths by fitting a Gaussian profile to the observed line. The focus was poor at $\lambda>8000 \AA$ and produced asymmetric emission lines. Line strengths in that spectral region are measured by summing the flux across a continuum-subtracted window that includes the entire line. The Balmer and $\mathrm{Ca}$ II $\mathrm{H}$ and $\mathrm{K}$ lines are bright from every source and are measured directly from the spectra. For all other lines we subtract a scaled photospheric template to increase our sensitivity to small fluxes. These line equivalent widths and corresponding fluxes are relative to the photospheric template and may be offset by a small amount in equivalent width if line emission is present but undetected in the photospheric template. This method may also yield false weak detections if the depth of $\mathrm{Na}$ I or $\mathrm{Ca}$ II photospheric absorption features differs substantially from star to star. Weak Ca II IR triplet emission from several targets is only treated as real if all three of the lines are detected, unless the nondetection has a large upper limit on equivalent width. Our detection limits typically range from $\sim 0.1$ to $1.5 \AA$, depending on the match with a photospheric template and the signal-to-noise ratio. The equivalent widths in low-resolution spectra are affected by absorption and may differ from equivalent widths measured in high-resolution spectra because of different spectral regions used to calculate the continuum emission.

The sources with strongest veiling also show the strongest line emission. For example, Ca II IR triplet emission is detected from only CIDA 1, 2M 0414+2811, GM Tau, 2M 0441+2534, and S0518+2327. Similarly, the Paschen series is detected from only 


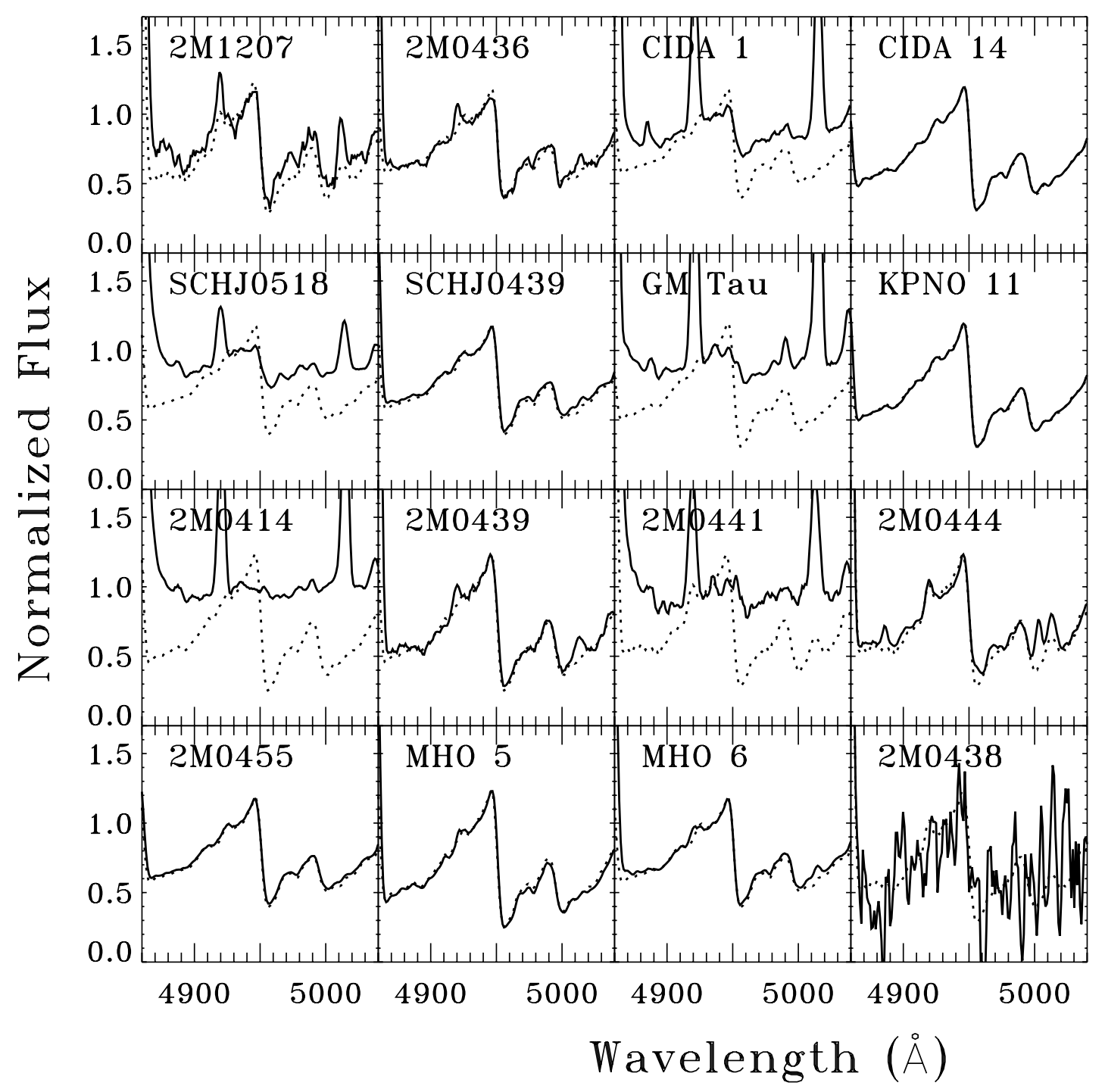

FIG. 3.- TiO absorption band at $4955 \AA$ used for veiling measurements.

CIDA 1, 2M 0414+2811, and GM Tau. Paschen lines blend with and contribute $\leq 10 \%$ of the flux in the Ca II IR triplet lines. Other lines, including the $\mathrm{Na}$ I D doublet, He II $\lambda 4686$, and $\mathrm{He}$ I lines, are detected from most stars with excess Balmer emission.

Optical forbidden lines, including the $[\mathrm{O} \mathrm{I}] \lambda \lambda 6300,6363$, [S II] $\lambda \lambda 6716,6731$, and [S I] $\lambda \lambda 4069,4076$ doublets, are detected from many sources. [N II] $\lambda 6584$ is detected from $2 \mathrm{M}$ $0444+2612$ but is difficult to resolve from the bright $\mathrm{H} \alpha$ emission for many other stars in the sample. The [O I] lines are unresolved in the cross-dispersion direction, which constrains the emission to have an FWHM of $<0.2-0.3$.

\subsection{Designation of Accretors from the Balmer Continuum}

Many emission-line diagnostics have been used to identify accretion onto young very low mass stars and brown dwarfs. An $\mathrm{H} \alpha$ equivalent width of $>10 \AA$ is the traditional criterion above which a young star is classified as an accretor. Against the weaker photospheric continua of redder $\mathrm{M}$ dwarfs, chromospheric emission can produce $\mathrm{H} \alpha$ emission with equivalent widths larger than $10 \AA$. In lieu of this diagnostic, the full width of the line profile at $10 \%$ the peak line flux in the $\mathrm{H} \alpha$ line has been used to diagnose accretion, with values greater than $180-200 \mathrm{~km} \mathrm{~s}^{-1}$ indicating accretion for very low mass stars (e.g., White \& Basri 2003; Muzerolle et al. 2003; Natta et al. 2004; Mohanty et al. 2005). Accretion onto low-mass stars has also been identified by emission in permitted optical lines. Optical forbidden lines are produced by outflows and can therefore be used as indirect accretion diagnostics because outflows require ongoing or recent accretion (e.g., Hamann 1994; Hartigan et al. 1995). The presence of excess Balmer and Paschen continuum emission is also associated with accretion (e.g., Valenti et al. 1993; Gullbring et al. 1998). In this subsection we describe the Balmer jump and excess Balmer continuum emission as an accretion diagnostic for the individual stars in our sample. In general, the classification of stars as accretors or nonaccretors based on the presence or absence of Balmer continuum emission is consistent with previous classifications using alternate accretion diagnostics.

Two mid-M dwarfs in our sample, CFHT 7 and MHO 7, were previously classified as nonaccretors. For CFHT 7, Guieu et al. (2007) measured only weak $\mathrm{H} \alpha$ emission and found an absence of excess IR emission shortward of and including the Spitzer $24 \mu \mathrm{m}$ MIPS bandpass. For MHO 7, Muzerolle et al. (2003) measured weak $\mathrm{H} \alpha$ emission with a $10 \%$ width of $115 \mathrm{~km} \mathrm{~s}^{-1}$ and no excess $K$-band emission. These two stars have Balmer jumps of 0.36 and 0.34 , respectively. Both stars are chromospherically 


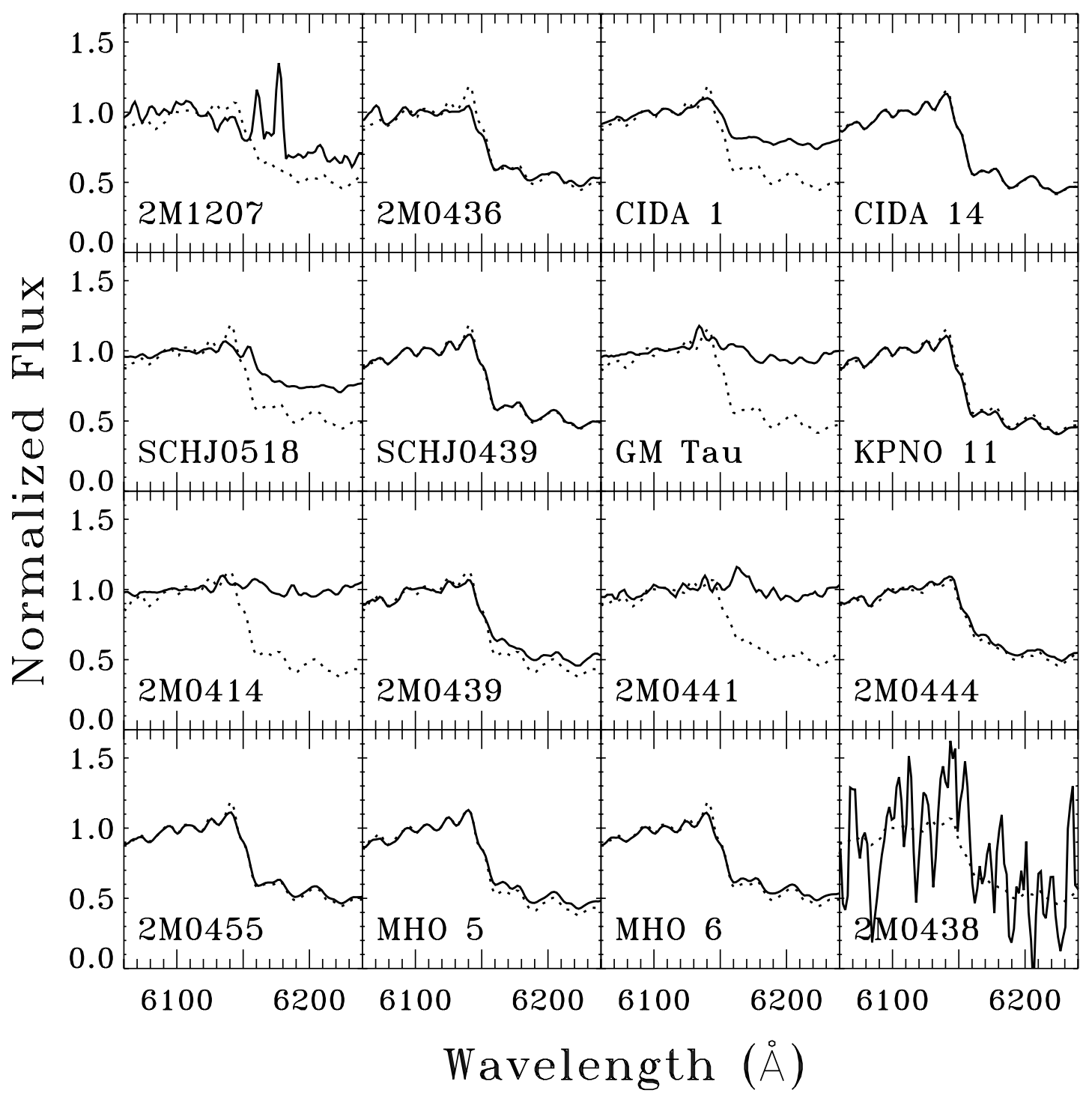

FIG. 4.- TiO absorption band at $6150 \AA ̊$ used for veiling measurements.

active, with equivalent widths in $\mathrm{Ca}$ II $\mathrm{H}$ and $\mathrm{K}$ and $\mathrm{He} \mathrm{I}$ lines that are larger than is typically measured from chromospheres of older, magnetically active M dwarfs (e.g., Giampapa et al. 1982; Gizis et al. 2002; Rauscher \& Marcy 2006; Allred et al. 2006). The earlier type nonaccretors $\mathrm{LkCa} 7$ and V819 Tau are bluer and have Balmer jumps of 0.44 and 0.46 , respectively.

Many stars in our LRIS sample have much larger observed Balmer jumps than those nonaccretors. The stars GM Tau, CIDA 1,

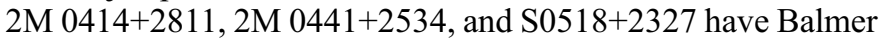
jumps ranging from 2.0 to 3.4 and excess Paschen continuum emission detected at $>6000 \AA$. These stars are also associated with strong emission in the Balmer series, $\mathrm{He}_{\mathrm{I}}$ lines, the Ca II IR triplet, $\mathrm{O}$ I $\lambda \lambda 7773,8446$, and many forbidden lines.

The stars MHO 6, 2M 0439+2544, $2 \mathrm{M} \mathrm{0444+2512,} \mathrm{S0439+}$

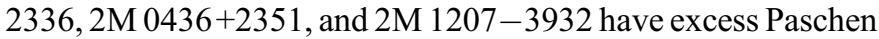
continuum emission detected at $4227 \AA$ but not at $>5500 \AA$. Five of these six stars have Balmer jumps between 0.7 and 1.4. The two observations of 2M 1207-3932 yield outlier Balmer jumps of 3.9 and 5.7. These six targets are associated with some strong permitted line emission, including bright Balmer continuum emission and $\mathrm{He}$ I line equivalent widths of $>1 \AA$, and some forbidden line emission. Any Ca II IR triplet emission is faint.
Each of these stars can be identified as accretors based on their high Balmer jump. Similarly, the higher mass stars TW Hya, DF Tau, RU Lupi, and DG Tau have Balmer jumps that are well above that of nonaccretors and can be classified as accretors based on either the Balmer jump or the presence of bright emission lines. As with other diagnostics, small Balmer jumps with values near those of nonaccreting templates may be inconclusive for classifying stars as accretors. V836 Tau has an observed Balmer jump of 0.60 , which is higher than nonaccretors with similar spectral type. Many young late $\mathrm{K}$ and early $\mathrm{M}$ stars that are not identified with accretion show no signs of excess Balmer continuum emission. Thus, this excess emission from V836 Tau is identified as a clear indication of accretion.

MHO 5, 2M 0455+3028, CIDA 14, KPNO 11, and V927 Tau all have observed Balmer jumps consistent with or slightly above the nonaccretors. Figure 6 shows that some excess Balmer emission relative to photospheric templates is present from each of these stars, with veilings at $3600 \AA$ that range from 0.14 to 0.54 (see Table 12). Emission-line diagnostics suggest that MHO 5, CIDA 14, and V927 Tau are actively accreting (see Appendix D), while the status of $2 \mathrm{M} 0455+3028$ and KPNO 11 is uncertain from other diagnostics. 
TABLE 4

Veiling Measurements of M Dwarfs from Keck Spectra

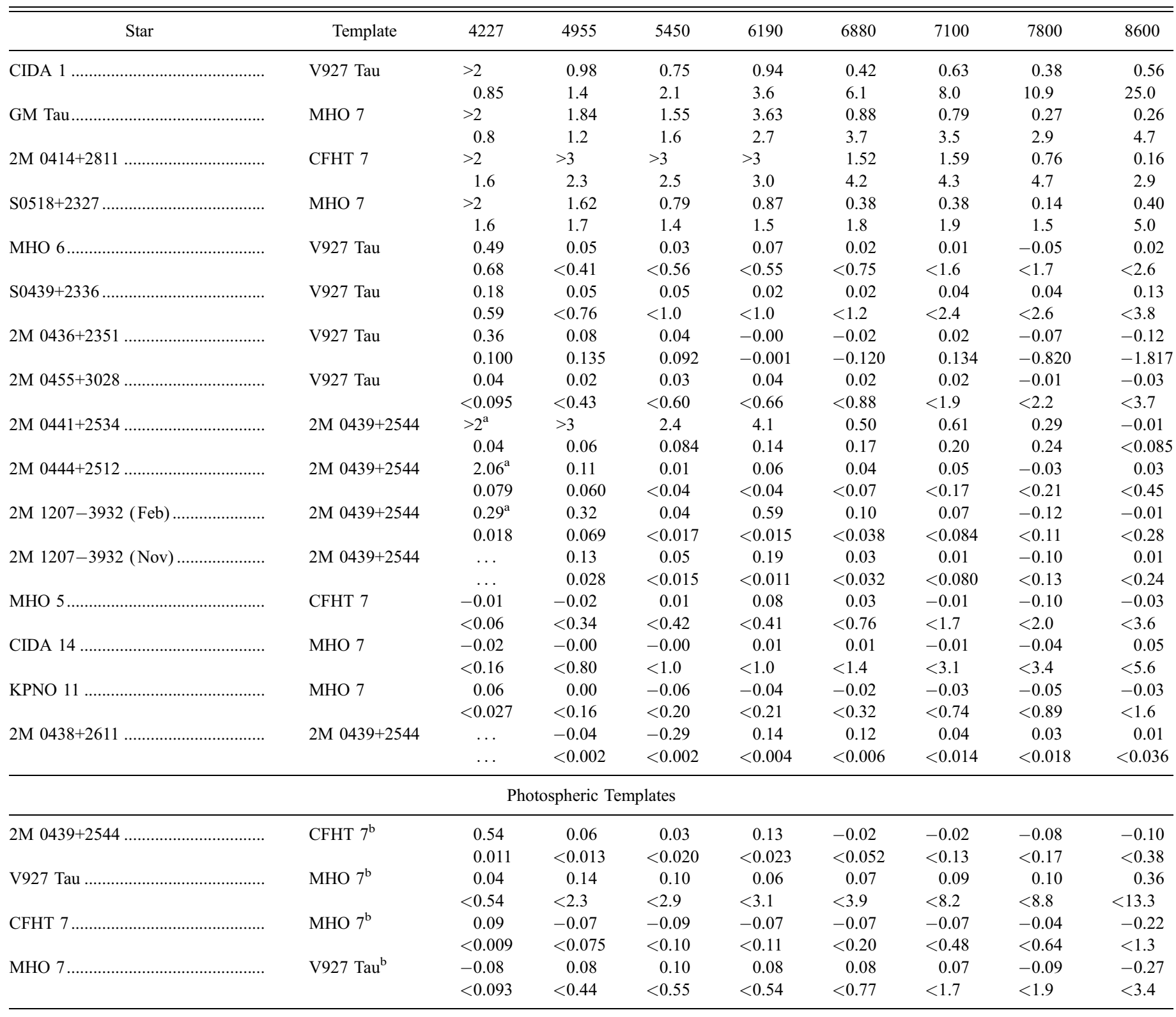

NотеS.-Veiling (accretion continuum flux divided by photospheric continuum flux) at listed wavelength is given in the top row and accretion continuum flux $\left(10^{-16} \mathrm{ergs} \mathrm{cm} \mathrm{cm}^{-2} \mathrm{~s}^{-1} \AA^{-1}\right)$ is given in the second row for each source. Flux upper limits are marked with a " $<$ " and are always calculated for $r_{\lambda}<0.05$. Stars are listed in order of $\dot{M}$ (see Table 12).

${ }^{a}$ CFHT 7 is used as a template instead of 2 M $0439+2544$ at $4227 \AA$.

${ }^{\mathrm{b}}$ Mismatched spectral template.

The weak excess Balmer continuum emission from these five stars could in principle be produced by the stellar chromosphere. Younger $\mathrm{M}$ dwarfs are bluer and more chromospherically active than older M dwarfs (Cardini \& Cassatella 2007), which is consistent with enhanced Balmer and Paschen continuum emission. However, the Balmer jump and elevated Balmer continuum emission are not detected during stellar flares (Hawley \& Pettersen 1991; Eason et al. 1992), which may suggest that stellar chromospheres do not produce any Balmer continuum emission. In this case, any weak excess emission could be attributed to accretion. A larger sample of $U$-band spectra from nonaccreting young $\mathrm{M}$ dwarfs is needed to definitively rule out a chromospheric origin. For the remainder of the paper we treat the excess emission from these five stars as upper limits. We also suggest the criterion that any observed Balmer jump of $>0.5$ for a mid-M dwarf should be considered an accretor.

\section{MASS ACCRETION RATES}

\subsection{Description of Accretion Models}

The accretion emission consists of a strong Balmer continuum, a weaker Paschen continuum, and many lines. The flux in the accretion continuum is measured for the entire 3000-9000 $\AA$ wavelength range from only the strongest three accretors (see Table 4). For the weakest accretors the veiling is measurable only shortward of the Balmer jump. Calculating the total luminosity of the 
TABLE 5

Veiling Measurements for Higher Mass Accretors Observed with $H S T$

\begin{tabular}{ccccc}
\hline \hline Star & Template & 4000 & 4600 & 5400 \\
\hline DF Tau (1999 Sep) ........ & LkCa 7 & $>5$ & 1.8 & 1.3 \\
& & 310 & 310 & 275 \\
DF Tau (2002 Aug)....... & LkCa 7 & $>5$ & 0.73 & 0.6 \\
& & 150 & 126 & 114 \\
RU Lup ................... & V819 Tau & $>5$ & 1.7 & 2.3 \\
& & 840 & 590 & 710 \\
DG Tau ..................... & V819 Tau & 3.0 & 1.4 & 1.7 \\
& & 37 & 59 & 64 \\
TW Hya (2000 May)..... & V819 Tau & 2.2 & 0.58 & 0.36 \\
& & 510 & 450 & 330 \\
TW Hya (2002 Jul) ........ & V819 Tau & 1.4 & 0.69 & 0.72 \\
& & 360 & 460 & 470 \\
V836 Tau ...................... & V819 Tau & 0.23 & $<0.1$ & $<0.08$ \\
& & 3.1 & $<4.9$ & $<4.0$ \\
\hline
\end{tabular}

Note.-Format same as in Table 4.
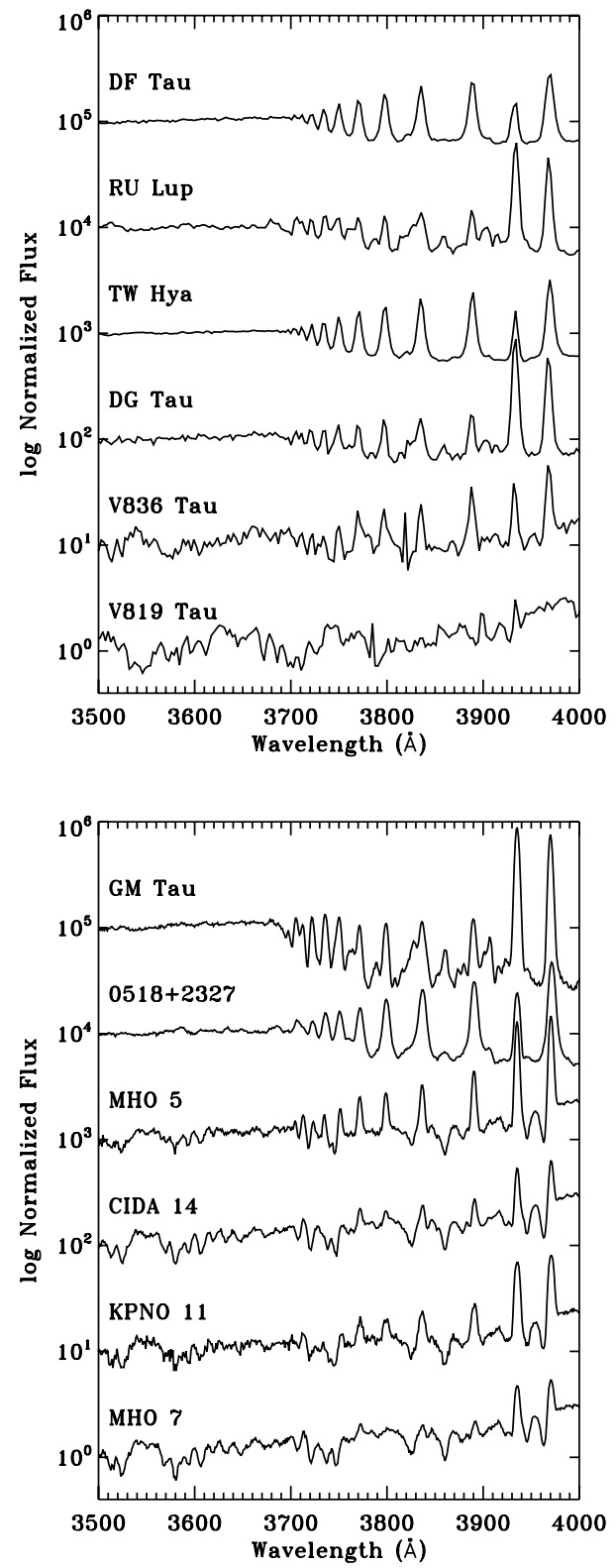

accretion shock requires modeling the observed accretion spectral energy distribution to obtain a bolometric correction that accounts for unseen emission.

The H Balmer and Paschen continua and series can be approximated by an isothermal, plane-parallel, pure hydrogen slab model following Valenti et al. (1993) and Gullbring et al. (1998). These simplistic models were developed based on the outdated boundary layer accretion paradigm but are robust to different geometries for the emitting gas. We use the isothermal planeparallel models from Valenti et al. (1993) because they does not rely on a specific morphology of the accretion flow and provides measurements of the accretion luminosity calculated with a methodology consistent with Valenti et al. (1993) and Gullbring et al. (1998).

The accretion slab models of Valenti et al. (1993) approximate the broadband hydrogen accretion continuum with the following free parameters: temperature $T$, density $n_{e}$, path length $L$ of the emitting gas (an opacity parameter), turbulent velocity, and
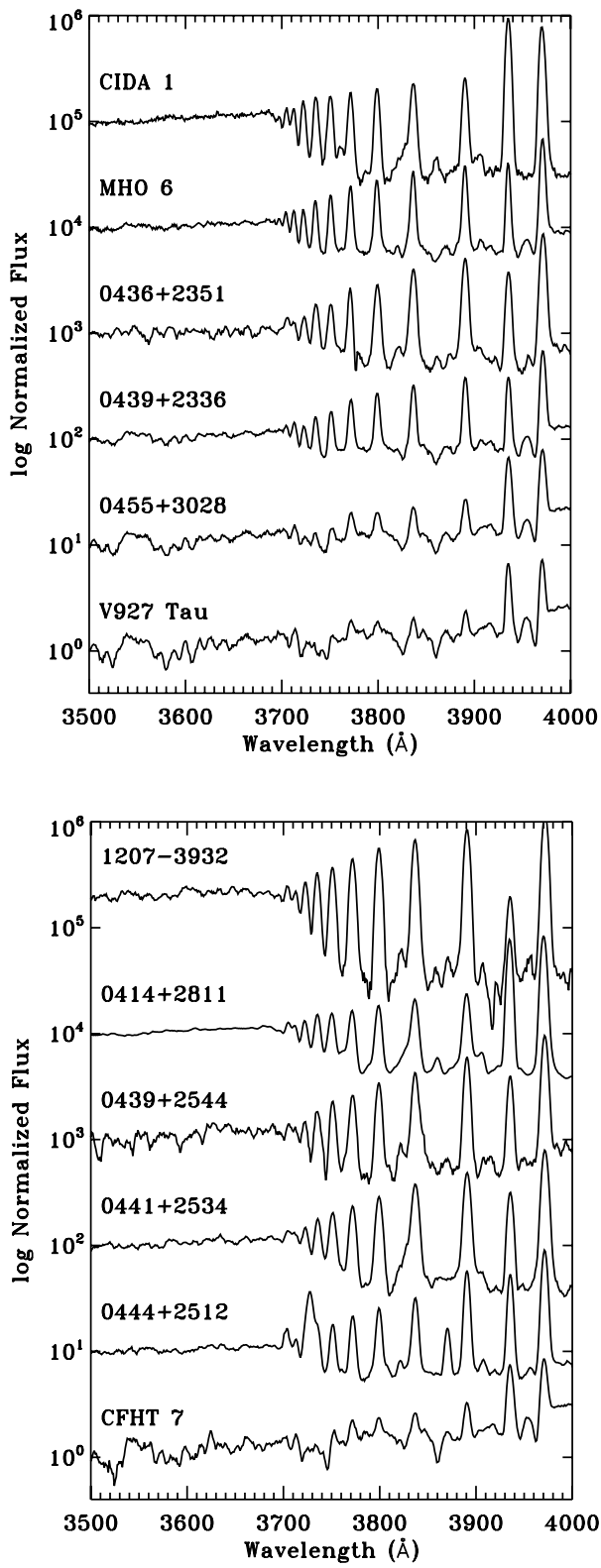

FIG. 5.- Spectral region from 3500 to 4000 Å for late K/early M stars (top left), M5 stars (top right), M5.5-M6 stars (bottom left), and M6.5-M8 stars (bottom right). The Balmer continuum, Balmer series, and $\mathrm{Ca}$ II $\mathrm{H}$ and $\mathrm{K}$ are apparent in emission. 
TABLE 6

Observed Properties of the Accretion Continuum

\begin{tabular}{|c|c|c|c|c|}
\hline Star & $J_{\text {obs }}{ }^{\mathrm{a}}$ & $J_{\bmod }^{\mathrm{b}}$ & $S_{B}^{\mathrm{c}}$ & $F_{4955} / F_{4227}{ }^{\mathrm{d}}$ \\
\hline \multicolumn{5}{|c|}{ Accretors in Keck I Sample } \\
\hline 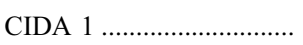 & 3.4 & $5.6 \pm 0.7$ & $4.1 \pm 2.0$ & 0.85 \\
\hline GM Tau............................ & 3.5 & $5.9 \pm 0.7^{\mathrm{e}}$ & $6.1 \pm 1.5$ & 0.9 \\
\hline 2M 0414+2811 ................... & 2.7 & $3.10 \pm 0.17$ & $8.1 \pm 1.0$ & 1.14 \\
\hline S0518+2327 ....................... & 2.1 & $2.35 \pm 0.15$ & $2.4 \pm 2.0$ & 0.83 \\
\hline 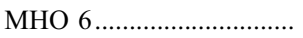 & 1.10 & $3.9 \pm 0.8$ & $7.7 \pm 1.5$ & $<0.5$ \\
\hline S0439+2336 ............................. & 0.76 & $3.1 \pm 1.5$ & $6.2 \pm 1.1$ & $<1.2$ \\
\hline $2 \mathrm{M} 0436+2351 \ldots \ldots \ldots \ldots \ldots$ & 1.48 & $3.8 \pm 1.2$ & $\ldots$ & $\ldots$ \\
\hline 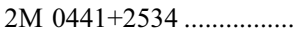 & 2.8 & $3.75 \pm 0.3$ & $7.2 \pm 1.0$ & 1.2 \\
\hline 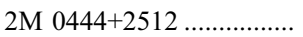 & 1.24 & $3.1 \pm 0.7$ & $6.6 \pm 1.2$ & 0.76 \\
\hline $2 \mathrm{M} 0439+2544 \ldots$ & 1.37 & $>3$ & $16 \pm 6$ & $\ldots$ \\
\hline $2 \mathrm{M} 1207-3932 \ldots$ & 5.9 & $15 \pm 4$ & $8.7 \pm 1.0$ & $\ldots$ \\
\hline 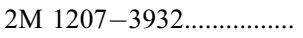 & 3.4 & $7 \pm 2$ & $10.7 \pm 2.0$ & $\ldots$ \\
\hline
\end{tabular}

Possible Accretors or Nonaccretors in Keck I Sample

\begin{tabular}{|c|c|c|c|c|}
\hline $2 \mathrm{M} 0455+3028 \ldots \ldots \ldots \ldots \ldots . . . . . .$. & 0.46 & $>1.3$ & $\ldots$ & ... \\
\hline 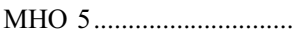 & 0.46 & $>1.5$ & $\ldots$ & $\ldots$ \\
\hline 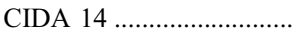 & 0.35 & $\ldots$ & $\ldots$ & $\ldots$ \\
\hline 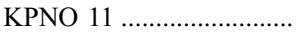 & 0.41 & $\ldots$ & $\ldots$ & $\ldots$ \\
\hline 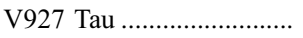 & 0.38 & $\ldots$ & $\ldots$ & $\ldots$ \\
\hline 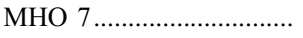 & 0.34 & $\ldots$ & $\ldots$ & $\ldots$ \\
\hline 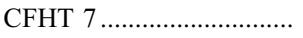 & 0.36 & $\ldots$ & $\ldots$ & $\ldots$ \\
\hline
\end{tabular}

Accretors in HST Sample

\begin{tabular}{|c|c|c|c|c|}
\hline DF Tau (1999 Sep).......... & 1.58 & $1.9 \pm 0.1$ & $5.4 \pm 0.4$ & $\ldots$ \\
\hline DF Tau (2002 Aug) .......... & 1.95 & $2.8 \pm 0.3$ & $5.0 \pm 0.4$ & $\ldots$ \\
\hline 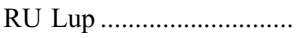 & 1.72 & $1.9 \pm 0.2$ & $2.9 \pm 0.5$ & . \\
\hline 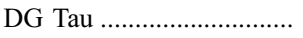 & 1.35 & $2.0 \pm 0.1$ & $5.2 \pm 0.6$ & $\ldots$ \\
\hline TW Hya (2000 May)....... & 1.66 & $2.3 \pm 0.2$ & $4.3 \pm 0.6$ & $\ldots$ \\
\hline TW Hya (2002 Jul) ......... & 2.0 & $3.1 \pm 0.5$ & $4.8 \pm 0.4$ & $\ldots$ \\
\hline 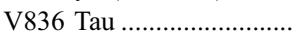 & 0.60 & $\ldots$ & $\ldots$ & $\ldots$ \\
\hline
\end{tabular}

Nonaccretors in HST Sample

\begin{tabular}{|c|c|c|c|c|}
\hline 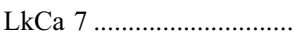 & 0.44 & $\ldots$ & $\ldots$ & $\ldots$ \\
\hline 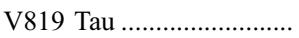 & 0.46 & $\ldots$ & $\ldots$ & $\ldots$ \\
\hline
\end{tabular}

a Observed flux ratio, $F_{4000} / F_{3600}$.

b Flux ratio, $F_{4000} / F_{3600}$, after photospheric subtraction and dereddening.

c Slope of Balmer continuum.

d Flux ratio as proxy for slope of Paschen continuum.

e Measured at alternate wavelength due to strong line blending.

filling factor $\delta$ of the accretion slab. At low density $\left(n_{e}<\right.$ $10^{13} \mathrm{~cm}^{-3}$ ) the Balmer jump is large and determined by the temperature of the emitting gas. At higher density stronger $\mathrm{H}^{-}$ continuum emission reduces the size of the Balmer jump and makes the continuum shape at $<3700 \AA$ bluer. The Balmer and Paschen continua become redder and the size of the Balmer jump increases with decreasing temperature, while both continua become bluer and the size of the Balmer jump decreases with increasing optical depth $\left(n_{e}\right.$ and $\left.L\right)$. In our fits we vary the temperature, density, and path length while setting the turbulent velocity to $\sim 120 \mathrm{~km} \mathrm{~s}^{-1}$, which is smaller than the $150 \mathrm{~km} \mathrm{~s}^{-1}$ used by Valenti et al. (1993). Larger turbulent velocities yield Balmer line profiles that are broader than is observed. Substantially smaller turbulent velocities are unable to reproduce the shoulder of the Balmer jump, located between the real Balmer jump at $3646 \AA$ and the observed Balmer jump at $\sim 3700 \AA$, that is attributed to blending of high Balmer lines. The synthetic spectrum is then scaled to the measured flux accretion continuum.
The filling factor of the emission on the stellar surface is calculated from the accretion luminosity and temperature.

Calvet \& Gullbring (1998) developed more advanced models of the pre- and postshock gas at the footpoint of the magnetospheric accretion column on the star. In their models the Balmer continuum is produced in the optically thin preshock gas while the Paschen continuum is produced in the optically thick postshock gas. The optically thin preshock gas has an intrinsically large Balmer jump. The optically thick postshock region has a high density and small Balmer jump. The observed Balmer jump is therefore not density sensitive but instead depends on the relative amount and temperature of the pre- and postshock gas. Our primary goal for modeling the measured accretion continuum is estimating bolometric corrections to convert the continuum flux to an accretion luminosity. Calvet \& Gullbring (1998) find that the two model variants lead to $2 \%-5 \%$ differences in the slope of the Balmer continuum and 5\%-10\% differences in the slope of the Paschen continua, so that the continuum emission from shock models is redder. However, our models and the models of Calvet \& Gullbring (1998) both successfully explain continuum emission at $<5600 \AA$ and both underestimate continuum emission at $>6000 \AA$ (see $\S 4.2$ ). We therefore suggest that any differences between the two models in the bolometric correction are modest.

\subsection{Application of Models to the Observed Spectra}

We fit the synthetic accretion spectrum plus a photospheric template to the unreddened blue spectra between 3200 and $5600 \AA$. The photospheric template is scaled to match our estimate for photospheric emission between 3200 and $5600 \AA$, which is informed by veiling estimates (see Tables 4 and 5).

The stars with measurable accretion continuum emission longward of the Balmer jump allow us to constrain some parameters of the plane-parallel slab. Figure 7 shows that the slope of the Balmer continuum constrains $T$ while the Balmer jump constrains $n_{e}$. Values of $T$ and $n_{e}$ for each star are adopted from the center of the acceptable contours, with $L$ subsequently tuned to match the strength of the high Balmer lines. Since $L$ and $n_{e}$ are not independent, a lower $L$ may be consistent with a larger $n_{e}$. In several cases $L$ could not be tuned to yield the observed flux in the high Balmer lines. In the context of the Calvet \& Gullbring (1998) shock models, the larger Balmer jump may be caused instead by fainter Paschen continuum emission from the optically thick postshock gas relative to the Balmer continuum from the optically thin preshock gas.

The lower Balmer lines are easily affected by emission or absorption in the wind and are consistently underestimated by our models. In magnetospheric accretion models of Muzerolle et al. (2001) and Kurosawa et al. (2006), emission in the Balmer lines is dominated by the funnel flow. If the high Balmer lines are also produced in the accretion flow, then the slab optical depths in our models should be larger. The slab optical depth may be smaller if instead the wind is optically thick and absorbs a large percentage of emission in all of the Balmer series lines.

The stars S0439+2336, 2M 0455+3028, MHO 5, CIDA 14, KPNO 11, and V836 Tau have weak Balmer continuum emission and undetected Paschen continuum emission. Since the size of the intrinsic Balmer jump for these targets is uncertain, we assume values for $n_{e}, T$, and $l$ based on our other data. We adjust the parameters to match the synthetic Balmer series and scale the emission so that the spectrum of a photospheric template plus accretion matches the total emission spectrum.

Table 12 lists approximate accretion slab parameters for each star, the estimated Balmer jump of the accretion continuum, 


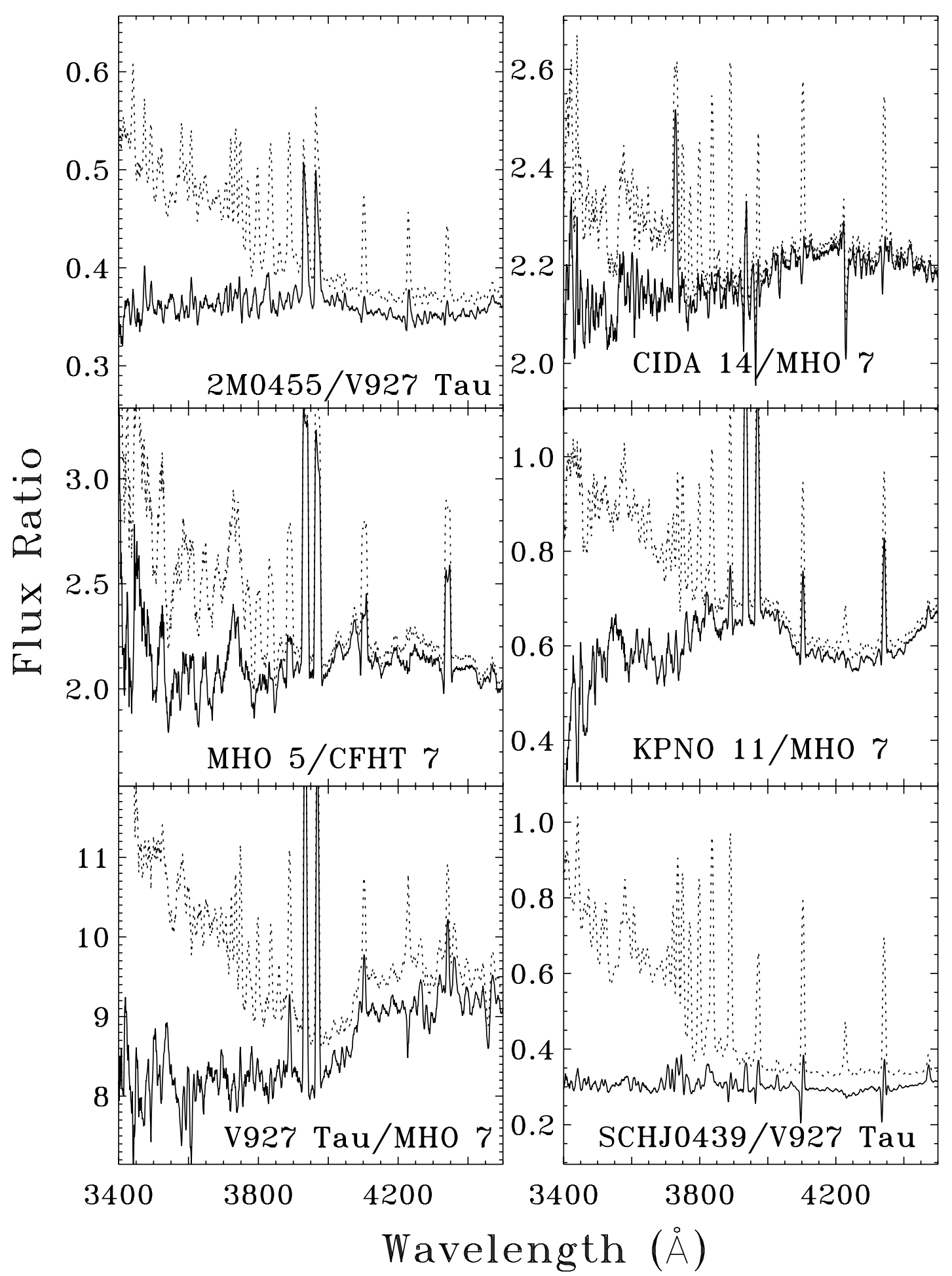

Fig. 6.-Balmer jump region for stars with weak or no accretion. The flux ratio of an observed spectrum to a photospheric template (dotted line; both smoothed by 11 pixels) increases at $\sim 3700$ when accretion is present. The solid line shows the same ratio, after subtracting excess Balmer line and continuum emission from the spectrum. For a perfect model and photospheric template, the residuals should be a line equal to the flux ratio with noise introduced from both the spectrum and photospheric template. Some excess Balmer continuum emission is seen from each source plotted here. The excess Balmer continuum emission from S0439+2336 is strong enough to be attributed to accretion. Fainter Balmer excess emission may be produced by either chromospheric activity or accretion.

the slope of the Balmer continuum, the veiling at 3600 and $4000 \AA$ measured from these fits, and the total accretion slab luminosity. The continuum emission from each target is well characterized by temperatures of $\sim 7000-9500 \mathrm{~K}$, densities of $(0.3-2) \times 10^{14} \mathrm{~cm}^{-3}$, and lengths of $\sim(0.5-50) \times 10^{7} \mathrm{~cm}$. In Figure 8 we show our fits to each spectrum. The synthetic hydrogen continuum is consistent with veiling measurements at $<5600 \AA$ but underestimates the veiling at $>6000 \AA$ from $2 \mathrm{M} 0414+2811$, $2 \mathrm{M} 0441+2534$, CIDA $1, \mathrm{GM}$ Tau, and S $0518+2327$ by a factor of 1.2-2. The intrinsic Balmer jump of 2M 1207-3932 (15 \pm 4 and $7 \pm 2$ for our two observations) is particularly large, which indicates a low $\mathrm{H}^{-}$opacity. A few outliers in the Valenti et al. (1993) and Gullbring et al. (1998) data also have large Balmer jumps. 
TABLE 7

Balmer Line Equivalent Widths and Fluxes

\begin{tabular}{|c|c|c|c|c|c|c|c|c|c|c|c|}
\hline Star & $\mathrm{H} \alpha$ & $\mathrm{H} \beta$ & $\mathrm{H} \gamma$ & $\mathrm{H} \delta$ & H6 & $\mathrm{H} 7$ & $\mathrm{H} 8$ & H9 & $\mathrm{H} 10$ & H11 & $\mathrm{H} 12$ \\
\hline 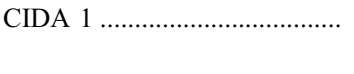 & $\begin{array}{l}232 \\
31.2\end{array}$ & $\begin{array}{r}134 \\
3.0\end{array}$ & $\begin{array}{l}80 \\
1.08\end{array}$ & $\begin{array}{l}64 \\
0.65\end{array}$ & $\begin{array}{l}41 \\
0.38\end{array}$ & $\begin{array}{l}49 \\
0.38\end{array}$ & $\begin{array}{l}37 \\
0.29\end{array}$ & $\begin{array}{l}27 \\
0.24\end{array}$ & $\begin{array}{l}18 \\
0.21\end{array}$ & $\begin{array}{l}17 \\
0.20\end{array}$ & $\begin{array}{l}10 \\
0.14\end{array}$ \\
\hline 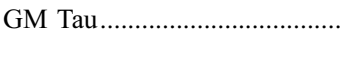 & $\begin{array}{l}281 \\
14.6\end{array}$ & $\begin{array}{l}68 \\
1.11\end{array}$ & $\begin{array}{l}39 \\
0.41\end{array}$ & $\begin{array}{l}30 \\
0.24\end{array}$ & $\begin{array}{l}8.8 \\
0.12\end{array}$ & $\begin{array}{l}26 \\
0.21\end{array}$ & $\begin{array}{l}13 \\
0.13\end{array}$ & $\begin{array}{l}11 \\
0.11\end{array}$ & $\begin{array}{l}12 \\
0.15\end{array}$ & $\begin{array}{l}11 \\
0.14\end{array}$ & $\begin{array}{l}9.1 \\
0.12\end{array}$ \\
\hline 2M 0414+2811 ......................... & $\begin{array}{r}280 \\
15\end{array}$ & $\begin{array}{r}103 \\
2.5\end{array}$ & $\begin{array}{l}72 \\
1.3\end{array}$ & $\begin{array}{l}58 \\
0.97\end{array}$ & $\begin{array}{l}30 \\
0.57\end{array}$ & $\begin{array}{l}32 \\
0.53\end{array}$ & $\begin{array}{l}23 \\
0.39\end{array}$ & $\begin{array}{l}17 \\
0.32\end{array}$ & $\begin{array}{c}10.6 \\
0.26\end{array}$ & $\begin{array}{l}8.7 \\
0.22\end{array}$ & $\begin{array}{l}5.6 \\
0.15\end{array}$ \\
\hline 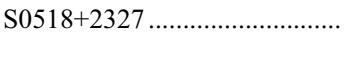 & $\begin{array}{l}50 \\
2.5\end{array}$ & $\begin{array}{l}67 \\
1.51\end{array}$ & $\begin{array}{l}60 \\
1.27\end{array}$ & $\begin{array}{l}54 \\
1.11\end{array}$ & $\begin{array}{l}28 \\
0.69\end{array}$ & $\begin{array}{l}27 \\
0.62\end{array}$ & $\begin{array}{l}19 \\
0.43\end{array}$ & $\begin{array}{l}9.8 \\
0.26\end{array}$ & $\begin{array}{l}5.3 \\
0.18\end{array}$ & $\begin{array}{l}4.7 \\
0.16\end{array}$ & $\begin{array}{l}1.9 \\
0.069\end{array}$ \\
\hline 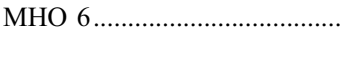 & $\begin{array}{l}55 \\
10.2\end{array}$ & $\begin{array}{l}31 \\
1.6\end{array}$ & $\begin{array}{l}37 \\
1.00\end{array}$ & $\begin{array}{l}28 \\
0.73\end{array}$ & $\begin{array}{l}27 \\
0.45\end{array}$ & $\begin{array}{l}27 \\
0.41\end{array}$ & $\begin{array}{l}21 \\
0.32\end{array}$ & $\begin{array}{l}16 \\
0.26\end{array}$ & $\begin{array}{c}12.1 \\
0.19\end{array}$ & $\begin{array}{l}9.6 \\
0.16\end{array}$ & $\begin{array}{l}5.3 \\
0.097\end{array}$ \\
\hline 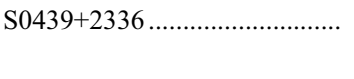 & $\begin{array}{l}13 \\
3.9\end{array}$ & $\begin{array}{l}18 \\
1.55\end{array}$ & $\begin{array}{l}24 \\
1.24\end{array}$ & $\begin{array}{l}18 \\
0.90\end{array}$ & $\begin{array}{l}21 \\
0.60\end{array}$ & $\begin{array}{l}16 \\
0.47\end{array}$ & $\begin{array}{l}14 \\
0.39\end{array}$ & $\begin{array}{l}11 \\
0.31\end{array}$ & $\begin{array}{l}8.9 \\
0.22\end{array}$ & $\begin{array}{l}6.2 \\
0.16\end{array}$ & $\begin{array}{l}2.7 \\
0.078\end{array}$ \\
\hline 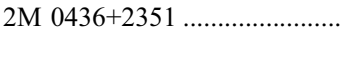 & $\begin{array}{r}28 \\
0.97\end{array}$ & $\begin{array}{l}47 \\
0.48\end{array}$ & $\begin{array}{l}74 \\
0.38\end{array}$ & $\begin{array}{l}62 \\
0.29\end{array}$ & $\begin{array}{l}46 \\
0.19\end{array}$ & $\begin{array}{l}44 \\
0.16\end{array}$ & $\begin{array}{l}32 \\
0.106\end{array}$ & $\begin{array}{l}16 \\
0.064\end{array}$ & $\begin{array}{l}10.2 \\
0.046\end{array}$ & $\begin{array}{l}9.2 \\
0.046\end{array}$ & $\begin{array}{l}5.7 \\
0.028\end{array}$ \\
\hline 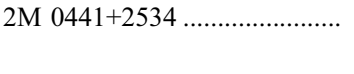 & $\begin{array}{r}586 \\
1.7\end{array}$ & $\begin{array}{r}228 \\
0.16\end{array}$ & $\begin{array}{l}158 \\
0.085\end{array}$ & $\begin{array}{l}135 \\
0.061\end{array}$ & $\begin{array}{l}72 \\
0.036\end{array}$ & $\begin{array}{l}70 \\
0.029\end{array}$ & $\begin{array}{l}47 \\
0.020\end{array}$ & $\begin{array}{l}29 \\
0.014\end{array}$ & $\begin{array}{l}14.6 \\
0.0101\end{array}$ & $\begin{array}{l}9.1 \\
0.0070\end{array}$ & $\begin{array}{l}3.9 \\
0.0035\end{array}$ \\
\hline 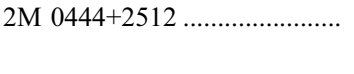 & $\begin{array}{l}100 \\
1.7\end{array}$ & $\begin{array}{r}126 \\
0.34\end{array}$ & $\begin{array}{l}94 \\
0.15\end{array}$ & $\begin{array}{l}62 \\
0.085\end{array}$ & $\begin{array}{l}49 \\
0.058\end{array}$ & $\begin{array}{l}27 \\
0.031\end{array}$ & $\begin{array}{l}20 \\
0.022\end{array}$ & $\begin{array}{l}18 \\
0.018\end{array}$ & $\begin{array}{l}10.3 \\
0.012\end{array}$ & $\begin{array}{c}\text { B } \\
\ldots\end{array}$ & $\begin{array}{c}\text { B } \\
\ldots\end{array}$ \\
\hline 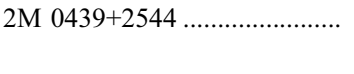 & $\begin{array}{r}158 \\
1.7\end{array}$ & $\begin{array}{l}93 \\
0.12\end{array}$ & $\begin{array}{l}93 \\
0.054\end{array}$ & $\begin{array}{r}87 \\
0.035\end{array}$ & $\begin{array}{l}73 \\
0.022\end{array}$ & $\begin{array}{l}50 \\
0.016\end{array}$ & $\begin{array}{l}44 \\
0.012\end{array}$ & $\begin{array}{l}36 \\
0.0093\end{array}$ & $\begin{array}{l}36 \\
0.0079\end{array}$ & $\begin{array}{l}18 \\
0.0056\end{array}$ & $\begin{array}{l}6.4 \\
0.0027\end{array}$ \\
\hline 2M 1207-3932 (Feb) ............ & $\begin{array}{l}396 \\
3.7\end{array}$ & $\begin{array}{r}341 \\
0.53\end{array}$ & $\begin{array}{l}286 \\
0.27\end{array}$ & $\begin{array}{l}323 \\
0.20\end{array}$ & $\begin{array}{r}142 \\
0.124\end{array}$ & $\begin{array}{r}146 \\
0.099\end{array}$ & $\begin{array}{r}132 \\
0.084\end{array}$ & $\begin{array}{l}89 \\
0.066\end{array}$ & $\begin{array}{l}55 \\
0.050\end{array}$ & $\begin{array}{l}27 \\
0.037\end{array}$ & $\begin{array}{l}16 \\
0.027\end{array}$ \\
\hline 2M 1207-3932 (Nov) ............ & $\begin{array}{l}200 \\
\quad 1.21\end{array}$ & $\begin{array}{l}210 \\
0.19\end{array}$ & $\begin{array}{l}234 \\
0.119\end{array}$ & $\begin{array}{r}132 \\
0.088\end{array}$ & $\begin{array}{l}\ldots \\
0.071\end{array}$ & $\begin{array}{l}\cdots \\
0.052\end{array}$ & 0.042 & $\begin{array}{c}\cdots \\
0.037\end{array}$ & $\begin{array}{l}\ldots \\
0.024\end{array}$ & $\begin{array}{c}\ldots \\
0.0106\end{array}$ & $\begin{array}{c}\cdots \\
0.008\end{array}$ \\
\hline 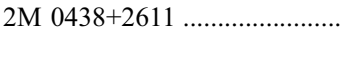 & $\begin{array}{l}99 \\
0.16\end{array}$ & $\begin{array}{l}158 \\
0.023\end{array}$ & $\begin{array}{r}622 \\
0.013\end{array}$ & $\begin{array}{c}\cdots \\
0.0056\end{array}$ & $\begin{array}{c}\ldots \\
0.0052\end{array}$ & $\begin{array}{c}\ldots \\
<0.0036\end{array}$ & $<0.0036$ & $<0.0024$ & $<0.0014$ & $\begin{array}{c}\cdots \\
<0.0014\end{array}$ & $\begin{array}{c}\cdots \\
<0.0014\end{array}$ \\
\hline 2M $0455+3028 \ldots \ldots \ldots \ldots \ldots \ldots \ldots$ & $\begin{array}{l}9.6 \\
2.12\end{array}$ & $\begin{array}{l}9.2 \\
0.45\end{array}$ & $\begin{array}{l}8.6 \\
0.26\end{array}$ & $\begin{array}{l}6.1 \\
0.15\end{array}$ & $\begin{array}{l}7.5 \\
0.095\end{array}$ & $\begin{array}{l}7.1 \\
0.082\end{array}$ & $\begin{array}{l}4.0 \\
0.051\end{array}$ & $\begin{array}{l}4.5 \\
0.054\end{array}$ & $\begin{array}{l}3.0 \\
0.029\end{array}$ & $\begin{array}{l}2.0 \\
0.018\end{array}$ & $\begin{array}{l}<1.0 \\
<0.012\end{array}$ \\
\hline 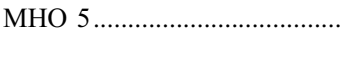 & $\begin{array}{l}43 \\
6.6\end{array}$ & $\begin{array}{l}30 \\
0.83\end{array}$ & $\begin{array}{l}19 \\
0.34\end{array}$ & $\begin{array}{c}13.4 \\
0.20\end{array}$ & $\begin{array}{l}13.1 \\
0.119\end{array}$ & $\begin{array}{l}10.4 \\
0.084\end{array}$ & $\begin{array}{l}6.5 \\
0.058\end{array}$ & $\begin{array}{l}5.7 \\
0.049\end{array}$ & $\begin{array}{l}5.2 \\
0.029\end{array}$ & $\begin{array}{l}2.8 \\
0.020\end{array}$ & $\begin{array}{l}2.1 \\
0.015\end{array}$ \\
\hline 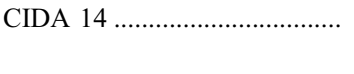 & $\begin{array}{r}10.2 \\
3.3\end{array}$ & $\begin{array}{l}7.5 \\
0.54\end{array}$ & $\begin{array}{l}3.9 \\
0.21\end{array}$ & $\begin{array}{l}3.4 \\
0.15\end{array}$ & $\begin{array}{l}3.8 \\
0.083\end{array}$ & $\begin{array}{l}4.5 \\
0.089\end{array}$ & $\begin{array}{l}1.4 \\
0.032\end{array}$ & $\begin{array}{l}2.2 \\
0.048\end{array}$ & $\begin{array}{c}<5.4 \\
0.07\end{array}$ & $\begin{array}{l}<3.8 \\
<0.05\end{array}$ & $\begin{array}{l}<3.8 \\
<0.05\end{array}$ \\
\hline 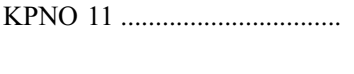 & $\begin{array}{c}13.6 \\
1.03\end{array}$ & $\begin{array}{c}12.5 \\
0.18\end{array}$ & $\begin{array}{l}9.5 \\
0.087\end{array}$ & $\begin{array}{l}7.6 \\
0.054\end{array}$ & $\begin{array}{l}8.4 \\
0.033\end{array}$ & $\begin{array}{l}7.4 \\
0.028\end{array}$ & $\begin{array}{l}3.3 \\
0.014\end{array}$ & $\begin{array}{l}3.6 \\
0.014\end{array}$ & $\begin{array}{l}3.5 \\
0.010\end{array}$ & $\begin{array}{l}<1.6 \\
<0.005\end{array}$ & $\begin{array}{l}<1.6 \\
<0.005\end{array}$ \\
\hline 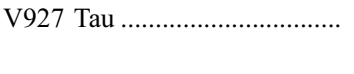 & $\begin{array}{l}10.7 \\
10.2\end{array}$ & $\begin{array}{l}7.9 \\
1.9\end{array}$ & $\begin{array}{l}3.7 \\
0.71\end{array}$ & $\begin{array}{l}3.2 \\
0.46\end{array}$ & $\begin{array}{l}3.6 \\
0.26\end{array}$ & $\begin{array}{l}3.9 \\
0.25\end{array}$ & $\begin{array}{l}1.6 \\
0.12\end{array}$ & $\begin{array}{l}2.2 \\
0.15\end{array}$ & $\begin{array}{l}2.1 \\
0.11\end{array}$ & $\begin{array}{l}<1.7 \\
<0.08\end{array}$ & $\begin{array}{l}<1.7 \\
<0.08\end{array}$ \\
\hline 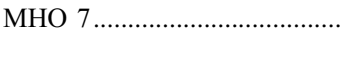 & $\begin{array}{l}7.4 \\
1.31\end{array}$ & $\begin{array}{l}5.4 \\
0.22\end{array}$ & $\begin{array}{l}2.5 \\
0.079\end{array}$ & $\begin{array}{l}1.9 \\
0.049\end{array}$ & $\begin{array}{l}2.0 \\
0.026\end{array}$ & $\begin{array}{l}<2.5 \\
<0.03\end{array}$ & $\begin{array}{l}<1.5 \\
<0.02\end{array}$ & $\begin{array}{l}1.6 \\
0.021\end{array}$ & $\begin{array}{l}<5.7 \\
<0.04\end{array}$ & $\begin{array}{l}<1.7 \\
<0.012\end{array}$ & $\begin{array}{l}<2.0 \\
<0.015\end{array}$ \\
\hline CFHT $7 \ldots \ldots \ldots \ldots \ldots \ldots \ldots \ldots \ldots \ldots$ & $\begin{array}{l}15 \\
0.63\end{array}$ & $\begin{array}{l}14 \\
0.084\end{array}$ & $\begin{array}{l}14 \\
0.042\end{array}$ & $\begin{array}{l}8.5 \\
0.021\end{array}$ & $\begin{array}{l}7.4 \\
0.0115\end{array}$ & $\begin{array}{l}5.3 \\
0.0082\end{array}$ & $\begin{array}{l}2.5 \\
0.004\end{array}$ & $\begin{array}{l}3.5 \\
0.005\end{array}$ & $\begin{array}{l}6.0 \\
0.0005\end{array}$ & $\begin{array}{c}\ldots \\
<0.003\end{array}$ & $\begin{array}{c}\ldots \\
<0.003\end{array}$ \\
\hline DF Tau (1999 Sep) ................... & $\begin{array}{l}\cdots \\
\ldots\end{array}$ & $\begin{array}{r}29 \\
150\end{array}$ & $\begin{array}{r}27 \\
109\end{array}$ & $\begin{array}{l}26 \\
90\end{array}$ & $\begin{array}{l}22 \\
68\end{array}$ & $\begin{array}{l}17 \\
54\end{array}$ & $\begin{array}{l}13 \\
42\end{array}$ & $\begin{array}{l}8.4 \\
30\end{array}$ & $\begin{array}{l}5.2 \\
19\end{array}$ & $\begin{array}{c}3.4 \\
13\end{array}$ & $\begin{array}{r}2.9 \\
11.5\end{array}$ \\
\hline DF Tau (2002 Aug) ................. & $\begin{array}{l}\cdots \\
\ldots\end{array}$ & $\begin{array}{l}26 \\
80\end{array}$ & $\begin{array}{l}20 \\
44\end{array}$ & $\begin{array}{l}17.7 \\
32\end{array}$ & $\begin{array}{l}16 \\
24\end{array}$ & $\begin{array}{l}15 \\
24\end{array}$ & $\begin{array}{l}10.9 \\
16\end{array}$ & $\begin{array}{r}7.8 \\
13.6\end{array}$ & $\begin{array}{r}6.1 \\
11.3\end{array}$ & $\begin{array}{l}3.9 \\
7.6\end{array}$ & $\begin{array}{l}4.2 \\
7.9\end{array}$ \\
\hline 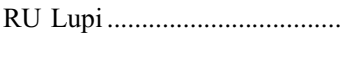 & $\begin{array}{l}\cdots \\
\ldots\end{array}$ & $\begin{array}{r}37 \\
360\end{array}$ & $\begin{array}{r}16 \\
160\end{array}$ & $\begin{array}{l}14.5 \\
113\end{array}$ & $\begin{array}{l}7.3 \\
68\end{array}$ & $\begin{array}{l}8.7 \\
74\end{array}$ & $\begin{array}{l}6.6 \\
57\end{array}$ & $\begin{array}{l}9.3 \\
80\end{array}$ & $\begin{array}{l}4.6 \mathrm{~B} \\
51 \mathrm{~B}\end{array}$ & $\begin{array}{l}4.4 \\
48\end{array}$ & $\begin{array}{l}3.0 \\
33\end{array}$ \\
\hline 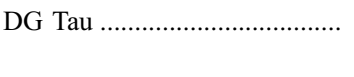 & $\begin{array}{l}\cdots \\
\ldots\end{array}$ & $\begin{array}{l}24 \\
29\end{array}$ & $\begin{array}{l}14 \\
11.5\end{array}$ & $\begin{array}{r}11.6 \\
7.0\end{array}$ & $\begin{array}{l}8.8 \\
4.2\end{array}$ & $\begin{array}{r}11.8 \\
5.1\end{array}$ & $\begin{array}{l}6.9 \\
3.1\end{array}$ & $\begin{array}{l}4.2 \\
2.1\end{array}$ & $\begin{array}{l}3.6 \\
1.9\end{array}$ & $\begin{array}{l}2.5 \\
1.4\end{array}$ & $\begin{array}{l}5.3 \\
2.5\end{array}$ \\
\hline TW Hya (2000 May)............... & $\begin{array}{l}\cdots \\
\cdots\end{array}$ & $\begin{array}{r}38 \\
470\end{array}$ & $\begin{array}{r}28 \\
280\end{array}$ & $\begin{array}{r}28 \\
230\end{array}$ & $\begin{array}{r}24 \\
160\end{array}$ & $\begin{array}{r}20 \\
137\end{array}$ & $\begin{array}{r}14 \\
104\end{array}$ & $\begin{array}{l}9.5 \\
76\end{array}$ & $\begin{array}{l}7.0 \\
57\end{array}$ & $\begin{array}{l}4.1 \\
37\end{array}$ & $\begin{array}{l}5.0 \\
42\end{array}$ \\
\hline TW Hya (2002 Jul) ................ & $\begin{array}{l}\cdots \\
\ldots\end{array}$ & $\begin{array}{r}77 \\
880\end{array}$ & $\begin{array}{r}48 \\
420\end{array}$ & $\begin{array}{r}42 \\
290\end{array}$ & $\begin{array}{r}33 \\
190\end{array}$ & $\begin{array}{r}26 \\
150\end{array}$ & $\begin{array}{r}18 \\
113\end{array}$ & $\begin{array}{l}12 \\
84\end{array}$ & $\begin{array}{l}7.6 \\
58\end{array}$ & $\begin{array}{l}5.3 \\
43\end{array}$ & $\begin{array}{l}3.6 \\
30\end{array}$ \\
\hline 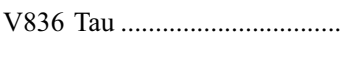 & $\begin{array}{l}\cdots \\
\ldots\end{array}$ & $\begin{array}{c}17 \\
9.6\end{array}$ & $\begin{array}{l}8.8 \\
3.1\end{array}$ & $\begin{array}{l}9.4 \\
2.0\end{array}$ & $\begin{array}{c}13 \\
1.3\end{array}$ & $\begin{array}{l}9.6 \\
0.84\end{array}$ & $\begin{array}{l}7.5 \\
0.70\end{array}$ & $\begin{array}{l}1.0 \\
0.85\end{array}$ & $\begin{array}{l}6.2 \\
0.45\end{array}$ & $\begin{array}{l}5.1 \\
0.35\end{array}$ & $\begin{array}{l}3.4 \\
0.25\end{array}$ \\
\hline 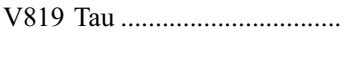 & $\begin{array}{l}\cdots \\
\cdots\end{array}$ & $\begin{array}{l}<0.8 \\
<1.3\end{array}$ & $\begin{array}{l}<2 \\
<1.6\end{array}$ & $\begin{array}{l}<1.8 \\
<0.9\end{array}$ & $\begin{array}{l}<4 \\
<0.8\end{array}$ & $\begin{array}{l}<7 \\
<0.6\end{array}$ & $\begin{array}{l}<2 \\
<0.4\end{array}$ & $\begin{array}{l}<6 \\
<1.0\end{array}$ & $\begin{array}{l}<6 \\
<0.6\end{array}$ & $\begin{array}{l}<6 \\
<0.8\end{array}$ & $\begin{array}{l}\cdots \\
\ldots\end{array}$ \\
\hline 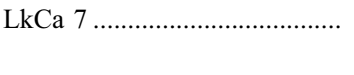 & $\begin{array}{l}\cdots \\
\ldots\end{array}$ & $\begin{array}{l}1.8 \\
4.8\end{array}$ & $\begin{array}{l}<3 \\
<6\end{array}$ & $\begin{array}{l}2.0 \\
2.2\end{array}$ & $\begin{array}{l}3.2 \\
1.5\end{array}$ & $\begin{array}{l}4.7 \\
1.6\end{array}$ & $\begin{array}{l}1.9 \\
0.86\end{array}$ & $\begin{array}{l}<5.5^{*} \\
<2.2^{*}\end{array}$ & $\begin{array}{l}<5 \\
<1.5\end{array}$ & $\begin{array}{l}<7 \\
<2.5\end{array}$ & $\begin{array}{l}\cdots \\
\ldots\end{array}$ \\
\hline
\end{tabular}

Notes._Equivalent width $(\AA)$ in first row, flux $\left(10^{-14} \mathrm{ergs}_{\mathrm{cm}}^{-2} \mathrm{~s}^{-1}\right)$ in second row for each star. B = blend; * $=$ marginal detection; ID = questionable line identification. 
TABLE 8

He Line Equivalent Widths and Fluxes

\begin{tabular}{|c|c|c|c|c|c|c|c|c|}
\hline Star & $\lambda 4026$ & $\lambda 4470$ & $\lambda 4922$ & $\lambda 5016$ & $\lambda 5876$ & $\lambda 6678$ & $\lambda 7065$ & He II $\lambda 4686$ \\
\hline \multicolumn{9}{|c|}{ Keck I LRIS Sample of Low-Mass Stars } \\
\hline \multirow[t]{2}{*}{ 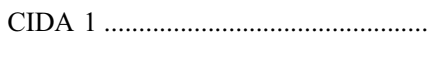 } & $4.3 \mathrm{~B}$ & 5.3 & 8.3 & 10.4 & 9.6 & 1.9 & 2.0 & 1.3 \\
\hline & $0.035 \mathrm{~B}$ & 0.086 & 0.22 & 0.26 & 0.49 & 0.22 & 0.31 & 0.23 \\
\hline \multirow[t]{2}{*}{ 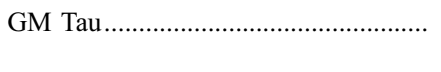 } & 2.5 & 5.0 & 13.9 & 16.1 & 4.6 & 1.1 & 0.5 & 0.2 \\
\hline & $0.019 \mathrm{~B}$ & $0.055 \mathrm{ID}$ & 0.23 & 0.26 & 0.12 & 0.052 & 0.030 & 0.015 \\
\hline \multirow[t]{2}{*}{ 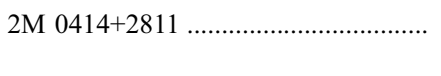 } & $2.8 \mathrm{~B}$ & 4.2 & 8.2 & 9.6 & 7.3 & 2.4 & 1.5 & 1.1 \\
\hline & $0.038 \mathrm{~B}$ & 0.085 & 0.20 & 0.24 & 0.23 & 0.11 & 0.084 & 0.062 \\
\hline \multirow{2}{*}{ 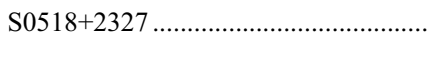 } & 3.5 & 5.7 & 2.8 & 3.4 & 7.7 & 2.4 & 0.9 & 1.5 \\
\hline & 0.064 & 0.12 & 0.071 & 0.078 & 0.19 & 0.087 & 0.041 & 0.033 \\
\hline \multirow[t]{2}{*}{ 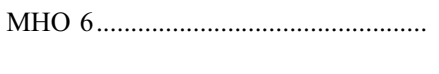 } & 1.9 & 2.1 & 0.6 & 0.9 & 2.9 & 0.5 & 0.6 & 0.5 \\
\hline & 0.047 & 0.082 & 0.041 & 0.044 & 0.19 & 0.060 & 0.10 & 0.023 \\
\hline \multirow{2}{*}{ 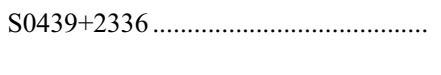 } & 1.2 & 1.2 & 0.3 & 0.4 & 1.5 & 0.4 & $<0.2$ & 0.1 \\
\hline & 0.054 & 0.094 & 0.033 & 0.038 & 0.18 & 0.084 & $<0.07$ & 0.011 \\
\hline \multirow[t]{2}{*}{ 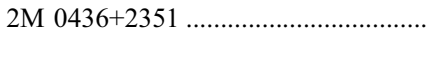 } & 3.8 & 3.8 & 0.95 & $<0.75^{*}$ & 2.9 & 1.7 & 2.3 & 0.84 \\
\hline & 0.018 & 0.028 & 0.012 & $<0.009^{*}$ & 0.052 & 0.036 & 0.103 & 0.0076 \\
\hline \multirow{2}{*}{ 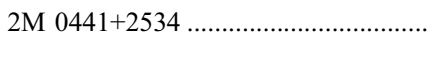 } & 8.1 & 16.2 & 6.8 & 7.5 & 29 & 8.5 & 3.6 & 2.1 \\
\hline & 0.0030 & 0.0084 & 0.0051 & 0.0058 & 0.041 & 0.021 & 0.013 & 0.0012 \\
\hline \multirow[t]{2}{*}{ 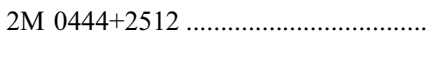 } & 3.9 & 7.2 & 1.5 & 4.9 & 12.4 & 1.4 & 0.7 & 2.1 \\
\hline & 0.0053 & 0.014 & 0.0063 & 0.014 & 0.054 & 0.014 & 0.013 & 0.0049 \\
\hline \multirow[t]{2}{*}{ 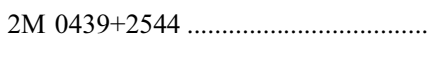 } & 4.6 & 8.7 & 1.0 & 2.3 & 11.2 & 1.8 & 0.5 & 1.5 \\
\hline & 0.0022 & 0.0062 & 0.0022 & 0.0031 & 0.028 & 0.013 & 0.0075 & 0.0015 \\
\hline \multirow{2}{*}{ 2M 1207-3932 (Feb).. } & 8.8 & 22 & 2.5 & 3.6 & 33 & 4.2 & 1.6 & 3.1 \\
\hline & 0.0074 & 0.021 & 0.0056 & 0.0057 & 0.075 & 0.022 & 0.014 & 0.0039 \\
\hline \multirow[t]{2}{*}{ 2M 1207-3932 (Nov) ......................... } & $<6.5$ & 267 & 3.7 & 4.5 & 21 & 3.5 & 1.2 & $<3.1$ \\
\hline & $<0.004^{*}$ & 0.017 & 0.0069 & 0.0053 & 0.038 & 0.015 & 0.010 & $<0.0034$ \\
\hline \multirow{2}{*}{ 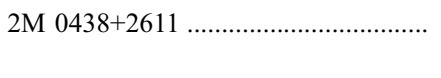 } & $\ldots$ & $\ldots$ & $\ldots$ & $\ldots$ & $<10$ & $<2$ & $<1.8$ & $\ldots$ \\
\hline & $<0.003$ & $<0.003$ & $<0.002$ & $<0.002$ & $<0.004$ & $<0.0015$ & $<0.0025^{*}$ & $<0.002$ \\
\hline 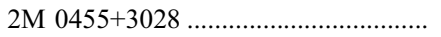 & 0.3 & $<0.17$ & 0.1 & 0.1 & 0.4 & $<0.13$ & $<0.3$ & $<0.08$ \\
\hline & 0.0081 & $<0.007^{*}$ & 0.0070 & 0.0056 & 0.030 & $<0.02 *$ & $<0.06$ & $<0.004$ \\
\hline 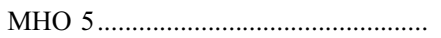 & $<0.8^{*}$ & $<1.0^{*}$ & $<0.4^{*}$ & $<0.4^{*}$ & 1.3 & $<0.25^{*}$ & $<0.16^{*}$ & $<0.25$ \\
\hline & $<0.014$ & $<0.020$ & $<0.022$ & $<0.042$ & 0.0555 & $<0.025$ & $<0.025$ & $<0.006$ \\
\hline 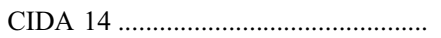 & $<0.3$ & $<0.18$ & $<0.1$ & 0.1 & 0.3 & $<0.07$ & $<0.1$ & $<0.10$ \\
\hline & $<0.015$ & $<0.012$ & $<0.012$ & $<0.009$ & 0.037 & $<0.016$ & $<0.04$ & $<0.007$ \\
\hline 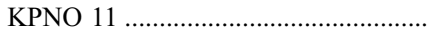 & 0.4 & 0.4 & 0.2 & $<0.18$ & 0.4 & 0.1 & $<0.2$ & $<0.10$ \\
\hline & 0.0033 & 0.0053 & 0.0059 & $<0.003$ & 0.0092 & 0.0061 & $<0.015$ & $<0.0015$ \\
\hline 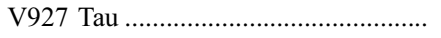 & $<0.15$ & $<0.4$ & $<0.15$ & $<0.02$ & 0.30 & $<0.2$ & $<0.2$ & $<0.25$ \\
\hline & $<0.02$ & $<0.08$ & $<0.06$ & $<0.06$ & 0.11 & $<0.12$ & $<0.18$ & $<0.06$ \\
\hline 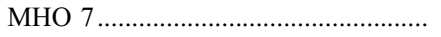 & $<0.18$ & $<0.33$ & $<0.25$ & $<0.30$ & $<0.20$ & $<0.27$ & $<0.17$ & $<0.2$ \\
\hline & $<0.005$ & $<0.013$ & $<0.02$ & $<0.014$ & $<0.012$ & $<0.035$ & $<0.035$ & $<0.008$ \\
\hline 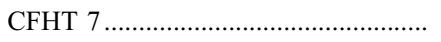 & $<0.45$ & $<0.4$ & 0.20 & $<0.26$ & 0.35 & 0.13 & $<0.3$ & $<0.4$ \\
\hline & $<0.0015$ & $<0.002$ & 0.0027 & $<0.002$ & 0.0042 & 0.0035 & $<0.015$ & $<0.002^{*}$ \\
\hline & & HST STI & ample of $\mathrm{H}$ & r Mass Sta & & & & \\
\hline 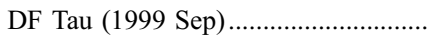 & 1.9 & 2.8 & 2.0 & 1.3 & $\ldots$ & $\ldots$ & $\ldots$ & 0.7 \\
\hline & 6.1 & 12.4 & 10.8 & 6.6 & $\ldots$ & $\ldots$ & $\ldots$ & 3.3 \\
\hline 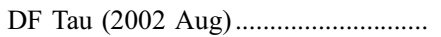 & 1.7 & 1.3 & 2.4 & 2.6 & $\ldots$ & $\ldots$ & $\ldots$ & 0.4 \\
\hline & 2.8 & 3.5 & 8.3 & 7.9 & $\ldots$ & $\ldots$ & $\ldots$ & 1.13 \\
\hline 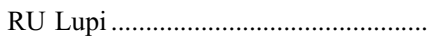 & $3.3 \mathrm{~B}$ & 3.3 & 9.5 & 9.5 & $\ldots$ & $\ldots$ & $\ldots$ & $<0.35^{*}$ \\
\hline & $25 \mathrm{~B}$ & 33 & 90 & 92 & $\ldots$ & $\ldots$ & $\ldots$ & $<3.2^{*}$ \\
\hline DG Tau & 1.3 & 1.7 & 3.2 & 4.4 & $\ldots$ & $\ldots$ & $\ldots$ & $<0.5$ \\
\hline & 0.65 & 1.5 & 3.9 & 5.0 & $\ldots$ & $\ldots$ & $\ldots$ & $<0.5$ \\
\hline TW Hya (2000 May)........................... & 2.2 & 2.8 & 0.9 & 1.0 & $\ldots$ & $\ldots$ & $\ldots$ & 1.1 \\
\hline & 16.8 & 29.8 & 10.9 & 11.8 & $\ldots$ & $\ldots$ & $\ldots$ & 14.1 \\
\hline 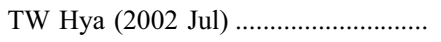 & 2.3 & 2.9 & 1.5 & 1.2 & $\ldots$ & $\ldots$ & $\ldots$ & 1.0 \\
\hline & 14.9 & 28 & 17 & 12.8 & $\ldots$ & $\ldots$ & $\ldots$ & 11.4 \\
\hline 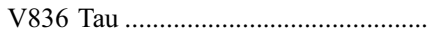 & 1.1 & 0.6 & 0.7 & 0.3 & $\ldots$ & $\ldots$ & $\ldots$ & 0.5 \\
\hline & 0.18 & 0.26 & 0.40 & 0.19 & $\ldots$ & $\ldots$ & $\ldots$ & 0.27 \\
\hline 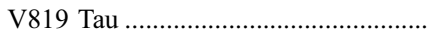 & $<2.5$ & $<1.5$ & $<1.4$ & $<0.7$ & $\ldots$ & $\ldots$ & $\ldots$ & $<1.0$ \\
\hline & $<1.0$ & $<1.5$ & $<2.0$ & $<1.0$ & $\ldots$ & $\ldots$ & $\ldots$ & $<1.5$ \\
\hline 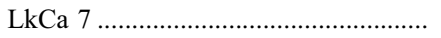 & $<2.8$ & $<1.5$ & $<1.2$ & $<0.4$ & $\ldots$ & $\ldots$ & $\ldots$ & $<1.2$ \\
\hline & $<2.0$ & $<2.5$ & $<3.0$ & $<1.0$ & $\ldots$ & $\ldots$ & $\ldots$ & $<3.0$ \\
\hline
\end{tabular}

Notes.-Equivalent width and line flux format as in Table 4. B = blend; ${ }^{*}=$ marginal detection; ID = questionable line identification. 
TABLE 9

Ca and Na Line Equivalent Widths and Fluxes

\begin{tabular}{|c|c|c|c|c|c|c|c|c|}
\hline Star & $\mathrm{Ca}$ II K & $\mathrm{Ca}$ II $\mathrm{H}^{\mathrm{a}}$ & Ca II $\lambda 8498$ & $\mathrm{Ca}$ II $\lambda 8542$ & Ca II $\lambda 8662$ & $\mathrm{Na}$ I $\lambda 5893$ & O I $\lambda 7773$ & O I $\lambda 8446$ \\
\hline \multirow[t]{2}{*}{ 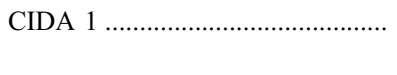 } & 175 & 160 & 14.7 & 18.9 & 14.4 & 10.4 & 1.7 & 2.5 \\
\hline & 1.49 & 1.32 & 7.8 & 10.4 & 9.1 & 0.48 & 0.54 & 1.36 \\
\hline \multirow[t]{2}{*}{ 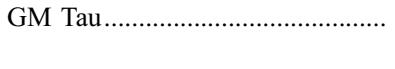 } & 106 & 135 & 28 & 29 & 21 & 15.4 & 1.14 & 3.4 \\
\hline & 1.35 & 1.15 & 4.4 & 4.5 & 4.1 & 0.41 & 0.114 & 0.55 \\
\hline \multirow{2}{*}{ 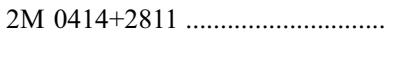 } & 116 & 142 & 39 & 40 & 30 & 11 & 1.24 & 2.0 \\
\hline & 1.9 & 2.3 & 4.9 & 5.4 & 5.1 & 0.35 & 0.108 & 0.28 \\
\hline \multirow[t]{2}{*}{ 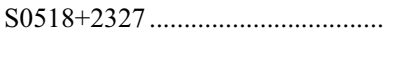 } & 20 & 59 & 1.73 & 2.1 & 1.94 & 4.35 & 2.30 & 1.23 \\
\hline & 0.43 & 1.21 & 0.22 & 0.27 & 0.32 & 0.093 & 0.19 & 0.15 \\
\hline \multirow[t]{2}{*}{ 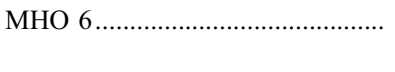 } & 30 & 43 & $<0.9$ & $<1.5$ & $<0.4$ & 0.98 & $<0.4$ & $<0.5$ \\
\hline & 0.45 & 0.92 & $<0.4$ & $<0.7$ & $<0.3$ & 0.048 & $<0.14$ & $<0.24$ \\
\hline \multirow[t]{2}{*}{ 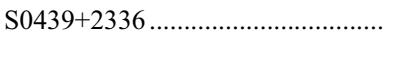 } & 23 & 35 & $<0.08$ & $<0.15$ & $<0.09$ & $<0.36$ & 0.16 & $<0.17$ \\
\hline & 0.59 & 1.21 & $<0.06$ & $<0.11$ & $<0.08$ & $<0.04$ & 0.083 & $<0.13$ \\
\hline \multirow[t]{2}{*}{ 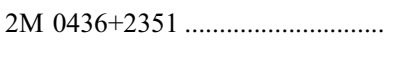 } & 41 & 79 & 1.7 & 1.6 & 1.1 & 2.2 & 1.1 & $<0.5$ \\
\hline & 0.13 & 0.35 & 0.15 & 0.16 & 0.12 & 0.039 & 0.065 & $<0.5$ \\
\hline \multirow{2}{*}{ 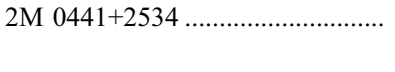 } & 53 & 160 & 6.6 & 6.4 & 5.0 & 11.4 & 1.14 & 1.29 \\
\hline & 0.021 & 0.062 & 0.094 & 0.107 & 0.123 & 0.016 & 0.0090 & 0.019 \\
\hline \multirow{2}{*}{ 2M $0444+2512 \ldots \ldots \ldots \ldots \ldots \ldots \ldots \ldots \ldots$} & 49 & 83 & $<0.6^{*}$ & 0.45 & 0.29 & 20 & $<0.4$ & $<0.6$ \\
\hline & 0.053 & 0.101 & $<0.04^{*}$ & 0.030 & 0.028 & 0.068 & $<0.016$ & $<0.05$ \\
\hline \multirow{2}{*}{ 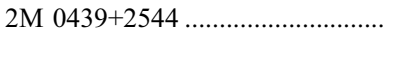 } & 38 & 93 & $<0.8$ & $<1.1$ & $<0.8$ & 4.8 & $<0.4$ & $<1.1$ \\
\hline & 0.013 & 0.037 & $<0.05$ & $<0.07$ & $<0.08$ & 0.0088 & 0.013 & $<0.08$ \\
\hline \multirow{2}{*}{ 2M 1207-3932 (Feb) ..................... } & 36.36 & 196.32 & $<0.7$ & $<1.6$ & $<0.8$ & 5.4 & 2.02 & 1.95 \\
\hline & 0.024 & 0.178 & $<0.03$ & $<0.076$ & $<0.05$ & 0.013 & 0.048 & 0.082 \\
\hline \multirow{2}{*}{ 2M 1207-3932 (Nov) .................... } & 90 & 200 & $<1.3$ & $<0.9$ & $<0.9$ & 4.7 & $<0.9$ & $<1.3$ \\
\hline & 0.012 & 0.071 & $<0.06$ & $<0.05$ & $<0.03$ & 0.008 & $<0.02$ & $<0.06$ \\
\hline \multirow[t]{2}{*}{ 2M $0438+2611 \ldots \ldots \ldots \ldots \ldots \ldots \ldots \ldots \ldots$} & $\ldots$ & 34 & $<1.3$ & $<1.2$ & $<0.8$ & $<17$ & $<0.9$ & $<0.9$ \\
\hline & $\ldots$ & 0.0053 & $<0.008$ & $<0.008$ & $<0.007$ & $<0.007$ & $<0.003$ & $<0.005$ \\
\hline \multirow[t]{2}{*}{ 2M $0455+3028 \ldots \ldots \ldots \ldots \ldots \ldots \ldots \ldots \ldots$} & 30 & 26 & $<0.3$ & $<0.3$ & 0.31 & 0.16 & $<0.11$ & $<0.35$ \\
\hline & 0.37 & 0.43 & $<0.21$ & $<0.23$ & 0.28 & 0.009 & $<0.049$ & $<0.25$ \\
\hline \multirow[t]{2}{*}{ 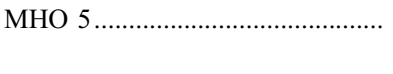 } & 48 & 42 & $<1.3$ & $<0.8$ & $<0.7$ & 1.6 & $<0.2$ & $<0.5$ \\
\hline & 0.43 & 0.51 & $<0.8$ & $<0.5$ & $<0.6$ & 0.046 & $<0.07$ & $<0.3$ \\
\hline \multirow[t]{2}{*}{ 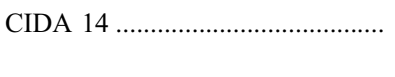 } & 14.3 & 11.9 & $<0.10$ & $<0.15$ & $<0.10$ & 0.86 & $<0.12$ & $<0.15$ \\
\hline & 0.30 & 0.34 & $<0.10$ & $<0.14$ & $<0.13$ & 0.057 & 0.080 & $<0.22$ \\
\hline \multirow{2}{*}{ 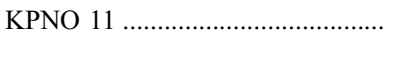 } & 32 & 26 & $<0.25$ & $<0.31$ & 0.28 & $<0.20$ & $<0.12$ & $<0.26$ \\
\hline & 0.125 & 0.143 & $<0.07$ & $<0.09$ & 0.123 & $<0.004$ & $<0.021$ & $<0.08$ \\
\hline \multirow[t]{2}{*}{ 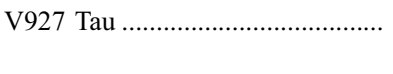 } & 24 & 18.4 & $<0.21$ & $<0.17$ & $<0.13$ & $<0.20$ & $<0.13$ & $<0.34$ \\
\hline & 1.55 & 1.63 & $<0.5$ & $<0.4$ & $<0.40$ & $<0.06$ & $<0.31$ & $<0.9$ \\
\hline \multirow[t]{2}{*}{ 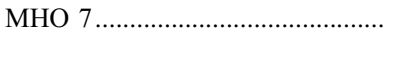 } & 13 & 10 & $<0.2$ & $<0.2$ & $<0.2$ & $<0.2$ & $<0.2$ & $<0.4$ \\
\hline & 0.17 & 0.18 & $<0.10$ & $<0.11$ & $<0.14$ & $<0.011$ & $<0.08$ & $<0.27$ \\
\hline \multirow{2}{*}{ 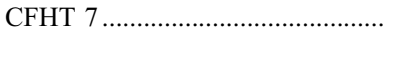 } & 27 & 21 & $<0.4$ & $<0.4$ & 0.21 & $<0.4$ & $<0.2$ & $<0.6$ \\
\hline & 0.043 & 0.047 & $<0.09$ & $<0.09$ & 0.064 & $<0.004$ & $<0.025$ & $<0.14$ \\
\hline
\end{tabular}

Notes.-Equivalent width and line flux format as in Table 4. B = blend; ${ }^{*}=$ marginal detection; ID = questionable line identification.

a Blended with $\mathrm{H} \epsilon$.

\subsection{Calculating Mass Accretion Rates and Errors}

The accretion luminosity, $L_{\text {acc }}$, can be converted to the mass accretion rate, $\dot{M}$, by assuming that the accretion energy is reprocessed entirely into the accretion continuum. The mass accretion rate is then

$$
\dot{M}=\left(1-\frac{R_{*}}{R_{\mathrm{in}}}\right)^{-1} \frac{L_{\mathrm{acc}} R_{*}}{G M_{*}} \sim 1.25 \frac{L_{\mathrm{acc}} R_{*}}{G M_{*}},
$$

where the factor $\left(1-R_{*} / R_{\text {in }}\right)^{-1} \sim 1.25$ is estimated by assuming that the accreting gas falls onto the star from the truncation radius of the disk, $R_{\text {in }} \sim 5 R_{*}$ (Gullbring et al. 1998). The stellar mass and radius are listed in Table 3 (see also Appendix C). We calculate mass accretion rates from $2 \times 10^{-12}$ to $10^{-8} M_{\odot} \mathrm{yr}^{-1}$ for the very low mass stars and brown dwarfs in our Keck LRIS sample and from $2 \times 10^{-10}$ to $5 \times 10^{-8} M_{\odot} \mathrm{yr}^{-1}$ in the HST STIS sample of higher mass stars (Table 12).

The parameters in equation (1) suffer from systematic and random uncertainties related to the data themselves, interpreta- tion of the data, and geometrical assumptions. In the following subsections and in Table 13 we describe for purposes of explicit clarity these sources of uncertainty and how they affect our measurements of $\dot{M}$.

\subsubsection{Errors in Assumed Stellar Properties}

Distance enters into calculations of $\dot{M}$ (eq. [1]) because $L_{\text {acc }} \propto$ $d^{2}$ and $R \propto d$, yielding $\dot{M} \propto d^{3}$. Based on the kinematic distances calculated by Bertout \& Genova (2006), the distance to any individual Taurus member can differ by $\sim 15 \mathrm{pc}$ from the standard $140 \mathrm{pc}$ distance (Kenyon et al. 1994). The $\sim 11 \%$ distance uncertainty in Taurus leads to a 0.13 dex in $\dot{M}$. The actual deviation in distance may not be random for our Taurus sample because the selection of targets here and in previous work is influenced by the optical brightness. Targets located in front of the cloud will be brighter because of a smaller distance modulus and lower extinctions.

Extinction also affects measures of $L_{\text {acc }}$ and $R$. The adopted 0.5 mag uncertainty in $A_{V}$ (see Appendix B) leads to a 0.32 dex 
TABLE 10

Wind Line Equivalent Widths and Fluxes

\begin{tabular}{|c|c|c|c|c|c|c|c|c|}
\hline Star & O I $\lambda 6300$ & O I $\lambda 6363$ & O I $\lambda 5577$ & $\mathrm{~S}$ II $\lambda 6716$ & S II $\lambda 6731$ & Fe II $\lambda 7155$ & S II $\lambda 4069$ & $\mathrm{~S}$ II $\lambda 4076$ \\
\hline \multirow[t]{2}{*}{ 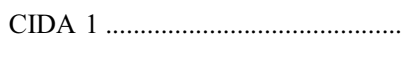 } & 1.17 & $<0.32$ & 0.48 & $<0.28$ & 0.21 & $<0.30^{*}$ & $<3.5 \mathrm{~B}$ & $<2.7 \mathrm{~B}$ \\
\hline & 0.084 & $<0.029$ & 0.0096 & $<0.030$ & 0.023 & $<0.042^{*}$ & $<0.034 \mathrm{~B}$ & $<0.028 \mathrm{~B}$ \\
\hline \multirow{2}{*}{ 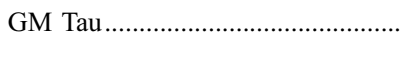 } & 5.4 & 2.0 & 1.9 & 0.34 & 0.64 & $<0.27^{*}$ & $<7.5 \mathrm{~B}$ & $<5.0 \mathrm{~B}$ \\
\hline & 0.19 & 0.077 & 0.052 & 0.015 & 0.030 & $<0.015^{*}$ & $<0.069 \mathrm{~B}$ & $<0.040 \mathrm{~B}$ \\
\hline \multirow{2}{*}{ 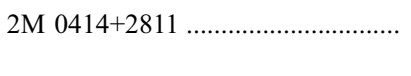 } & 3.2 & 1.17 & 1.01 & 0.42 & 0.17 & 0.15 & $<2.8 \mathrm{~B}$ & $<2.8 \mathrm{~B}$ \\
\hline & 0.12 & 0.047 & 0.033 & 0.020 & 0.0078 & 0.0081 & $<0.047 \mathrm{~B}$ & $<0.049 \mathrm{~B}$ \\
\hline \multirow{2}{*}{ 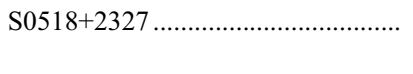 } & 1.02 & $0.44 \mathrm{~B}$ & 1.30 & $<0.21^{*}$ & $<0.17$ & 0.25 & $<0.63$ & $<1.47 \mathrm{~B}$ \\
\hline & 0.028 & $0.014 \mathrm{~B}$ & 0.035 & $<0.0069^{*}$ & $<0.0057$ & 0.0099 & $<0.0127$ & $<0.030 \mathrm{~B}$ \\
\hline \multirow[t]{2}{*}{ 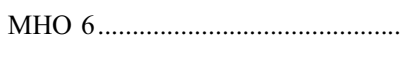 } & 0.88 & 0.17 & 0.18 & $<0.12$ & $<0.31$ & $<0.24$ & 6.0 & 2.2 \\
\hline & 0.075 & 0.021 & 0.021 & $<0.014$ & $<0.036$ & $<0.032$ & 0.15 & 0.051 \\
\hline \multirow{2}{*}{ 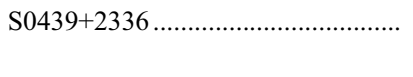 } & 0.46 & $<0.18$ & 0.17 & $<0.21$ & $<0.15$ & $<0.18$ & 0.68 & 0.53 \\
\hline & 0.068 & $<0.037$ & 0.028 & $<0.040$ & $<0.028$ & $<0.041$ & 0.031 & 0.024 \\
\hline \multirow[t]{2}{*}{ 2M $0436+2351 \ldots \ldots \ldots \ldots \ldots \ldots \ldots \ldots \ldots$} & $<1.0$ & $<0.3$ & $<1.4$ & $<1.5$ & $<0.2$ & $<0.7$ & $<2.0$ & $<2.0$ \\
\hline & $<0.015$ & $\ldots$ & $<0.019$ & $<0.03$ & $<0.005$ & $<0.02$ & $<0.011$ & $<0.011$ \\
\hline \multirow[t]{2}{*}{ 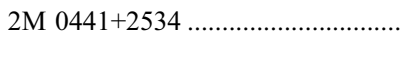 } & 8.3 & 2.3 & 4.3 & $<1.02^{*}$ & $<0.67^{*}$ & $<0.26$ & $<5.29$ & $<2.60$ \\
\hline & 0.015 & 0.0048 & 0.0011 & $<0.0026^{*}$ & $<0.0017^{*}$ & $<0.0008$ & $<0.0023$ & $<0.0012$ \\
\hline \multirow[t]{2}{*}{ 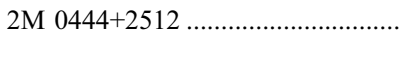 } & 62 & 13.2 & 6.6 & 16 & 29 & 0.68 & 413 & 129 \\
\hline & 0.40 & 0.13 & 0.038 & 0.13 & 0.27 & 0.0094 & 0.56 & 0.16 \\
\hline \multirow{2}{*}{ 2M $0439+2544 \ldots \ldots \ldots \ldots \ldots \ldots \ldots \ldots \ldots \ldots$} & 1.51 & $<0.36$ & 1.75 & $<0.93$ & $<0.18$ & $<0.18$ & $<1.39$ & $<1.66$ \\
\hline & 0.0060 & $<0.0023$ & 0.0071 & $<0.0059$ & $<0.0012$ & $<0.0016$ & $<0.0006$ & $<0.0008$ \\
\hline \multirow[t]{2}{*}{ 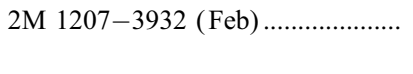 } & $<2.3$ & $<0.7$ & 2.4 & $<0.47$ & $<0.44$ & $<0.38$ & $<3.30$ & 7.1 \\
\hline & $<0.006$ & $<0.003$ & 0.0059 & $<0.0022$ & $<0.0022$ & $<0.0024$ & $<0.0026$ & 0.0062 \\
\hline \multirow{2}{*}{ 2M 1207-3932 (Nov) ....................... } & $<5.0$ & $<0.91$ & 1.6 & $<0.53$ & $<0.36$ & $<0.36$ & $<8.14$ & $<7.46$ \\
\hline & $<0.013$ & $<0.0035$ & 0.0015 & $<0.0021$ & $<0.0015$ & $<0.0020$ & $<0.0047$ & $<0.0048$ \\
\hline \multirow[t]{2}{*}{ 2M $0438+2611 \ldots \ldots \ldots \ldots \ldots \ldots \ldots \ldots \ldots$} & 98 & 20 & 20 & 5.0 & 8.6 & $<2.3$ & $\ldots$ & $\ldots$ \\
\hline & 0.048 & 0.0149 & 0.0052 & 0.0037 & 0.0072 & $<0.0023$ & 0.0106 & 0.0018 \\
\hline \multirow[t]{2}{*}{ 2M $0455+3028 \ldots \ldots \ldots \ldots \ldots \ldots \ldots \ldots \ldots \ldots \ldots \ldots \ldots \ldots \ldots \ldots \ldots \ldots$} & $<0.06$ & $<0.09$ & 0.27 & $<0.20$ & $<0.11$ & $<0.12$ & $<0.22$ & $<0.14$ \\
\hline & $<0.0066$ & $<0.0124$ & 0.0112 & $<0.028$ & $<0.0147$ & $<0.021$ & $<0.0052$ & $<0.0034$ \\
\hline \multirow[t]{2}{*}{ 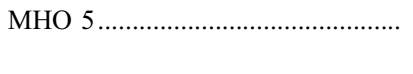 } & 1.64 & 0.35 & $<0.58$ & $<0.11^{*}$ & $<0.09$ & $<0.23$ & $<0.52$ & $<0.45$ \\
\hline & 0.101 & 0.033 & $<0.034$ & $<0.0103^{*}$ & $<0.0082$ & $<0.027$ & $<0.0083$ & $<0.0072$ \\
\hline \multirow[t]{2}{*}{ 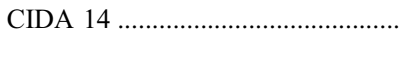 } & 0.27 & $<0.12$ & $<0.12$ & $<0.06$ & $<0.06^{*}$ & $<0.08$ & $<0.15$ & $<0.19$ \\
\hline & 0.040 & $<0.026$ & $<0.019$ & $<0.012$ & $<0.013^{*}$ & $<0.021$ & $<0.0068$ & $<0.0084$ \\
\hline \multirow[t]{2}{*}{ 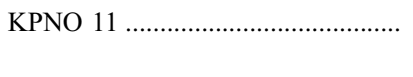 } & $<0.23$ & $<0.08$ & $<0.50$ & $<0.09$ & $<0.12$ & $<0.13$ & $<0.40$ & $<0.34$ \\
\hline & $<0.0073$ & $<0.0040$ & $<0.0145$ & $<0.0039$ & $<0.0056$ & $<0.0072$ & $<0.0029$ & $<0.0025$ \\
\hline \multirow[t]{2}{*}{ 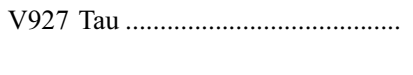 } & $<0.17$ & $<0.16$ & $<0.41$ & $<0.11$ & $<0.11$ & $<0.07$ & $<0.25$ & $<0.28$ \\
\hline & $<0.081$ & $<0.108$ & $<0.19$ & $<0.069$ & $<0.064$ & $<0.052$ & $<0.037$ & $<0.040$ \\
\hline \multirow{2}{*}{ 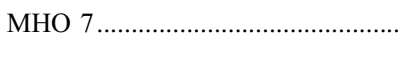 } & $<0.22$ & $<0.07$ & $<0.38$ & $<0.20$ & $<0.16$ & $<0.20$ & $<0.28$ & $<0.13$ \\
\hline & $<0.0175$ & $<0.0086$ & $<0.032$ & $<0.021$ & $<0.018$ & $<0.027$ & $<0.0073$ & $<0.0033$ \\
\hline \multirow[t]{2}{*}{ 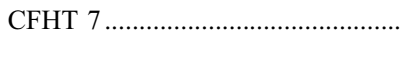 } & $<0.24$ & $<0.18$ & $<1.09$ & $<0.15$ & $<0.16$ & $<0.27$ & $<0.58$ & $<0.31$ \\
\hline & $<0.0042$ & $<0.0048$ & $<0.0156$ & $<0.0036$ & $<0.0041$ & $<0.0091$ & $<0.0016$ & $<0.0009$ \\
\hline
\end{tabular}

Notes.-Equivalent width and line flux format as in Table $4 . \mathrm{B}=$ blend; ${ }^{*}=$ marginal detection; ID $=$ questionable line identification.

uncertainty in $L_{\mathrm{acc}}$ and a 0.08 dex uncertainty in $R$ for a total of 0.4 dex uncertainty in $\dot{M}$. If $R_{V}>3.1$, then the extinction curve would be flatter and our $A_{V}$ would be underestimated.

The $\dot{M}$ measurements also depend on the ratio $R / M$, which is calculated from the effective temperature and theoretical evolutionary tracks (Appendix C). The temperature is uncertain by $\sim 75 \mathrm{~K}$, including both the uncertainty of roughly 0.5 spectral type subclasses and the conversion of spectral type to temperature (Appendix A). In the Baraffe et al. (1998) pre-main-sequence evolutionary tracks at $2 \mathrm{Myr}$ and with temperatures from Luhman et al. (2003) a difference of 0.5 spectral classes corresponds to $25 \%$ larger mass and a 10\% smaller radius. Alternate pre-mainsequence evolutionary tracks from D’Antona \& Mazzitelli (1998) would increase our mass estimates $1.1-1.7$ times larger than the Baraffe et al. (1998) tracks. White \& Hillenbrand (2004) suggest that evolutionary tracks underestimate stellar masses by $30 \%-50 \%$.

All sources except V927 Tau and DF Tau are assumed to be single. If any sources are binaries, the lower luminosity per star would imply that we have overestimated $R$. The shallower potential well would result in $\dot{M}$ being 1.4 times lower than is calculated here. In cases where both sources are accretors, the accretion luminosity and mass accretion rate are the total rate onto both stars.

\subsubsection{Uncertainty in Bolometric Corrections}

Converting the measured accretion flux into an accretion luminosity requires a bolometric correction for unseen emission (§ 4.2). However, the shape of the broadband accretion continuum is not well understood. Our single-temperature slab models underestimate the veiling at $6000-9000 \AA$ by factors of $1.2-2$, as also occurs for the multitemperature shock models (Calvet \& Gullbring 1998). The veiling at even longer wavelengths (e.g., White \& Hillenbrand 2004; Edwards et al. 2006) also seems larger than can be explained by the isothermal slab that fits well the Balmer continuum and short-wavelength Paschen continuum.

If we hypothetically double the accretion continuum flux at $\lambda>6000 \AA$, the total accretion luminosity increases by 0.15 dex. If instead the flux per $\AA$ is constant between 6000 and $20000 \AA$ (despite the presumed presence of the Paschen jump), then the accretion luminosity increases by 0.3 dex. A smaller uncertainty 
TABLE 11

Equivalent Width and Line Fluxes for HST Sample

\begin{tabular}{|c|c|c|c|c|}
\hline Star & $\mathrm{Ca}$ II $\mathrm{K}$ & $\mathrm{Ca}$ II $\mathrm{H}^{\mathrm{a}}$ & {$[\mathrm{O}$ I $] \lambda 5577$} & {$\left[\mathrm{~S}_{\mathrm{I}}\right] \lambda \lambda(4069+4076)^{\mathrm{b}}$} \\
\hline \multirow[t]{2}{*}{ 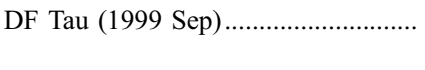 } & 10.4 & 30 & 0.7 & $<1.5$ \\
\hline & 31 & 92 & 6.6 & $<4.7$ \\
\hline \multirow[t]{2}{*}{ 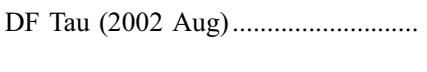 } & 28 & 38 & 0.9 & 3.0 \\
\hline & 42 & 56 & 4.1 & 4.7 \\
\hline \multirow[t]{2}{*}{ 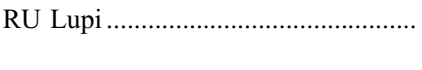 } & 50 & 39 & $<0.3$ & 9.2 \\
\hline & 500 & 330 & $<3$ & 72 \\
\hline \multirow[t]{2}{*}{ DG Tau } & 61 & 44 & 0.39 & $19^{c}$ \\
\hline & 33 & 22 & 0.67 & $9.2^{\mathrm{c}}$ \\
\hline \multirow{2}{*}{ TW Hya (2000 May).......................... } & 9.6 & 32.0 & $<0.15$ & $<0.8$ \\
\hline & 65 & 230 & $<2.5$ & $<6.0$ \\
\hline \multirow[t]{2}{*}{ TW Hya (2002 Jul) ............................ } & 11.5 & 45 & $<0.15$ & $<0.7$ \\
\hline & 65 & 270 & $<2.0$ & $<4.0$ \\
\hline \multirow{2}{*}{ 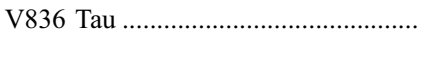 } & 12.9 & 19.5 & $<0.26^{*}$ & 4.1 \\
\hline & 1.37 & 2.6 & $<0.24^{*}$ & 0.79 \\
\hline \multirow[t]{2}{*}{ 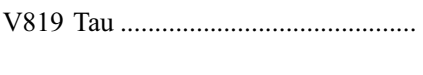 } & 4.1 & 4.2 & $<0.5$ & $<1.7$ \\
\hline & 0.95 & 1.2 & $<1.2$ & $<0.8$ \\
\hline \multirow[t]{2}{*}{ LkCa 7} & 7.9 & 7.0 & $<0.5$ & $<2.5$ \\
\hline & 3.8 & 4.2 & $<2.0$ & $<2.0$ \\
\hline
\end{tabular}

Notes.-Equivalent width and line flux format as in Table $4 . \mathrm{B}=$ blend; ${ }^{*}=$ marginal detection; ID = questionable line identification.

a Blended with $\mathrm{H} \epsilon$.

b Lines are marginally resolved and are co-added here.

c Emission extended by $0.3^{\prime \prime}$ in slit and included in extraction.

TABLE 12

Model Accretion Parameters

\begin{tabular}{|c|c|c|c|c|c|c|c|c|c|}
\hline Star & Template & $\begin{array}{c}T \\
(\mathrm{~K})\end{array}$ & $\begin{array}{c}n_{e} \\
\left(10^{14} \mathrm{~cm}^{-3}\right)\end{array}$ & $\begin{array}{c}L \\
\left(10^{7} \mathrm{~cm}\right)\end{array}$ & $\begin{array}{c}\delta \\
(\%)\end{array}$ & $r_{3600}$ & $r_{4000}$ & $\begin{array}{c}L_{\mathrm{acc}} \\
\left(10^{-4} L_{\odot}\right)\end{array}$ & $\begin{array}{c}\dot{M}_{\mathrm{acc}} \\
\left(10^{-10} M_{\odot} \mathrm{yr}^{-1}\right)\end{array}$ \\
\hline \multicolumn{10}{|c|}{ Low-Mass Accretors in Keck I Sample } \\
\hline 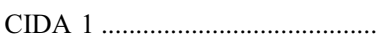 & V927 $\mathrm{Tau}^{\mathrm{a}}$ & 10500 & 0.8 & 10 & 1.01 & 70 & 4 & 280 & 120 \\
\hline 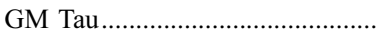 & MHO 7 & 9000 & 0.7 & 20 & 0.53 & 110 & 4.9 & 81 & 20 \\
\hline 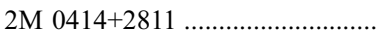 & CFHT 7 & 7400 & 2.5 & 2 & 3.3 & 200 & 20 & 32 & 9.0 \\
\hline 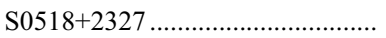 & MHO 7 & 10500 & 2.5 & 1 & 0.59 & 68 & 6.7 & 28 & 5.0 \\
\hline 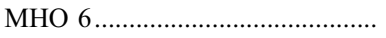 & V927 Tau & 7800 & 1.5 & 8 & 0.31 & 3.4 & 0.29 & 14 & 4.4 \\
\hline 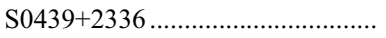 & V927 Tau & 8300 & 2.0 & 3 & 0.12 & 1.4 & 0.15 & 6.5 & 1.5 \\
\hline 2M $0436+2351 \ldots \ldots \ldots \ldots \ldots \ldots \ldots \ldots \ldots$ & V927 Tau & $(9500)$ & 1.3 & 1. & 1.7 & 5.0 & 0.35 & 3.8 & 0.53 \\
\hline 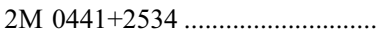 & CFHT 7 & 8000 & 1.5 & 5 & 0.38 & 40 & 3.9 & 0.67 & 0.19 \\
\hline 2M $0444+2512 \ldots \ldots \ldots \ldots \ldots \ldots \ldots \ldots \ldots$ & CFHT 7 & 8200 & 2.0 & 2.5 & 0.034 & 3.5 & 0.20 & 0.26 & 0.092 \\
\hline 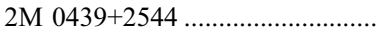 & CFHT 7 & $(7500)$ & $(1.0)$ & 20 & 0.19 & 3.4 & 0.14 & 0.17 & 0.069 \\
\hline 2M 1207-3932 (Feb) ................... & CFHT 7 & 7500 & 0.3 & 7 & 1.00 & 26 & 0.8 & 0.061 & 0.023 \\
\hline 2M 1207-3932 (Nov) ................ & CFHT 7 & 6500 & 1.5 & 20 & 1.4 & 21 & 0.7 & 0.044 & 0.017 \\
\hline \multicolumn{10}{|c|}{ Possible Accretors in Keck I Sample } \\
\hline $2 \mathrm{M} 0455+3028 \ldots \ldots \ldots \ldots \ldots \ldots \ldots \ldots \ldots$ & V927 Tau & $(9500)$ & $(2.0)$ & 1.5 & 0.007 & 0.4 & 0.06 & 1.7 & 0.58 \\
\hline 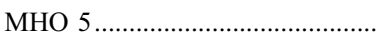 & CFHT $7^{\mathrm{b}}$ & $(8500)$ & $(2.0)$ & 2 & 0.006 & 0.22 & 0.02 & 0.46 & 0.22 \\
\hline 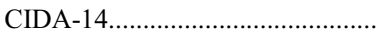 & MHO 7 & $(9500)$ & $(2.0)$ & 5 & 0.002 & 0.14 & 0.013 & 0.29 & 0.14 \\
\hline 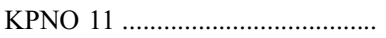 & $\mathrm{MHO} 7^{\mathrm{c}}$ & $(8500)$ & $(2.0)$ & 6 & 0.008 & 0.54 & 0.04 & 0.23 & 0.062 \\
\hline 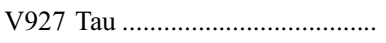 & MHO 7 & $(9500)$ & $(2.0)$ & 3 & 0.001 & 0.24 & 0.05 & $3.4^{\mathrm{d}}$ & $1.3^{\mathrm{d}}$ \\
\hline \multicolumn{10}{|c|}{ Accretors in HST Sample } \\
\hline DF Tau (Sep. 99) ....................... & $\mathrm{LkCa} 7$ & 8500 & 3.0 & 2.0 & 7.0 & 16 & 4 & 2400 & 460 \\
\hline 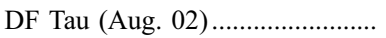 & $\mathrm{LkCa} 7$ & 9000 & 1.6 & 4.0 & 2.5 & 10 & 1.8 & 1200 & 230 \\
\hline 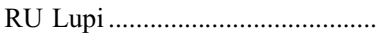 & V819 Tau & 9200 & 2.0 & 1.7 & 28 & 75 & 13 & 3500 & 180 \\
\hline 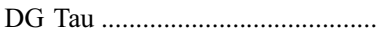 & V819 Tau & 8200 & 2.2 & 12 & 19 & 12 & 2.5 & 870 & 41 \\
\hline TW Hya (May 00) ........................ & V819 Tau & 9000 & 2.0 & 1.5 & 12 & 12 & 2.2 & 360 & 15.4 \\
\hline TW Hya (Jul. 02) ........................ & V819 Tau & 9000 & 1.8 & 1.0 & 12 & 12 & 1.7 & 340 & 14.6 \\
\hline 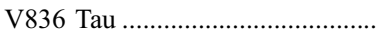 & V819 Tau & $(9000)$ & $(2.0)$ & 0.5 & 1.4 & 0.65 & 0.1 & 32 & 1.5 \\
\hline
\end{tabular}

a The photospheric template of V927 Tau is adjusted slightly by adding the spectrum of MHO 7.

${ }^{\mathrm{b}}$ Increased the flux of the template CFHT 7 by assuming $A_{V}=1$ to account for the spectral type discrepancy.

c Decreased the flux of the template MHO 7 by assuming $A_{V}=0.3$ to account for the spectral type discrepancy.

d Total $L_{\text {acc }}$ and $\dot{M}$ for both components. 

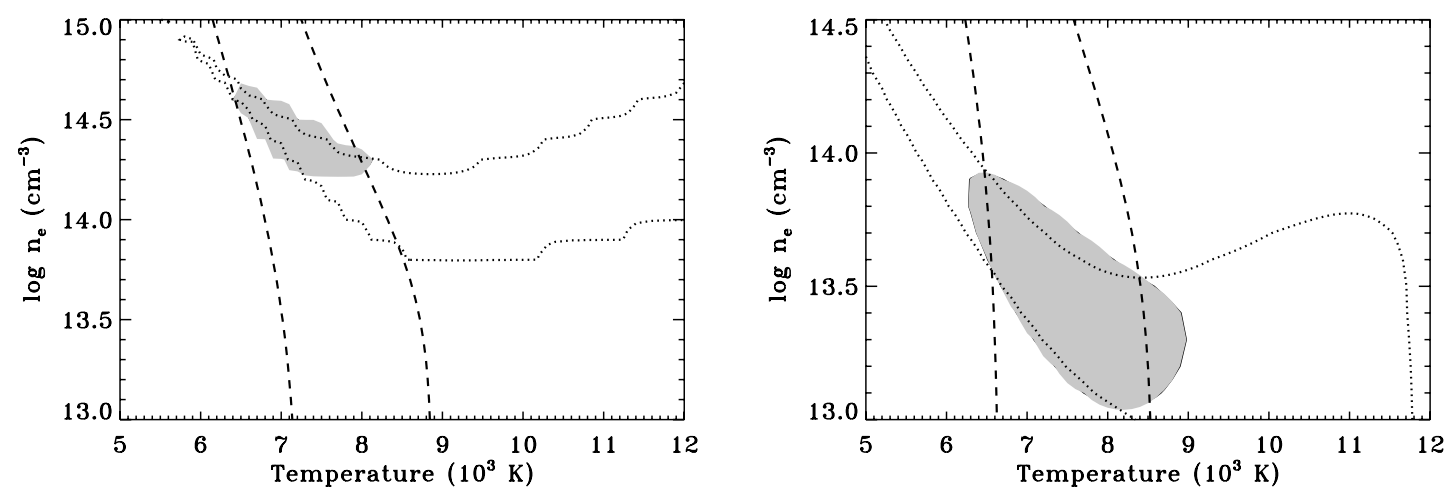

FIg. 7.-Contours of acceptable $T$ and $n_{e}$ for isothermal hydrogen slab model fits to the spectra of 2M 0414+2811 (left) and 2M 1207-3932 (right). The vertical contours that determine $T$ are constrained by the slope of the Balmer continuum. The horizontal contours that determine $n_{e}$ are constrained by the size of the Balmer jump. For $2 \mathrm{M} 0414+2811$ the acceptable values of $T$ and $n_{e}$ together differ from their intersection because they are only acceptable for different values of $L$.

of $\sim 0.1$ dex in $L_{\text {acc }}$ is introduced by the uncertainty in temperature $(\sim 1000-2000 \mathrm{~K})$ of the plane-parallel slab.

\subsubsection{Exclusion of Line Emission}

Our $\dot{M}$ estimates rely on accurate calculations of the total accretion energy that escapes from the shock. Such emission is seen in both continuum and line emission, but we include only the total continuum emission from the accretion slab in estimating $L_{\text {acc }}$. The observed Balmer line fluxes (Table 7) total 0.2 times $L_{\text {acc }}$ for most of the accretors but are roughly equivalent to $L_{\text {acc }}$ for the M7-M8 accretors (see Tables 7 and 12). The formation of and flux in lower Balmer lines are complicated by emission and absorption in the accretion funnel flow and in outflows. In principle, other lines (e.g., Ca II IR triplet, Lyman series, far-ultraviolet and $\mathrm{X}$-ray lines) may also need to be included. For TW Hya, X-ray and UV lines account for $\sim 30 \%-45 \%$ of the total accretion flux, most of which is in H I Ly $\alpha$ (Kastner et al. 2002; Herczeg et al. 2004). Our exclusion of line emission from the accretion luminosity is consistent with previous $L_{\text {acc }}$ estimates and is essential for simplifying these calculations and comparisons with existing $\dot{M}$ estimates.

\subsubsection{Geometric Assumptions}

In the magnetospheric accretion paradigm, gas accretes from the inner disk truncation radius, $R_{\mathrm{in}}$, onto the star along magnetic field lines, where $R_{\text {in }}$ is determined by where the magnetic field intercepts the disk. Gullbring et al. (1998) noted that infall energy depends on $R_{\text {in }}$ and therefore included the factor of (1$R_{*} / R_{\text {in }}$ ) in equation (1). The $R_{\text {in }}$ ranges from $3 R_{*}$ to $10 R_{*}$ based on magnetic field strengths of higher mass accretors (Johns-Krull 2007). We assume $R_{\text {in }}=5 R_{*}$ for all targets in this sample for consistency with the same assumption made by Gullbring et al. (1998). A factor of 2 error in $R_{*} / R_{\text {in }}$ leads to a $\sim 0.05-0.12$ dex error in the mass accretion rate.

As the gas accretes along the magnetic field lines and shocks at or near the stellar photosphere, some of the emission from the hot spot will be directed at the star. We follow Valenti et al. (1993) and Gullbring et al. (1998) in assuming that all of the accretion energy escapes from the star as hydrogen continuum emission. By this assumption, any accretion emission directed at the star either is coherently reradiated by the star or provides some heating to the nearby gas, which then cools by hydrogen continuum emission. Hartigan et al. (1995) and Hartigan \& Kenyon (2003) instead assumed that half of the Balmer and Paschen continuum emission is absorbed by the star, which increases the calculated $\dot{M}$ by a factor of 2 . The accretion luminosity may also not be isotropic.
The accretion hot spot is assumed to be the source for all Balmer and Paschen continuum emission from our sample. No excess emission is seen in a sample of nonaccreting $\mathrm{K}$ dwarfs that serve as templates for the higher mass accretors discussed here. Thus, the weak excess Balmer emission from V836 Tau that is attributable to accretion can be accurately measured. Whether the faint excess Balmer emission from 2M 0455+3028, CIDA 14, MHO 5, KPNO 11, and V927 Tau is produced by accretion or may be chromospheric is uncertain. Using V927 Tau as a template may lead us to underestimate the upper limit of $\dot{M}$ onto $2 \mathrm{M} 0455+3028$ by $0.2 \mathrm{dex}$. Weak Balmer continuum emission in the template spectrum has a negligible effect for targets with larger $L_{\mathrm{acc}} / L_{\mathrm{bol}}$.

\subsubsection{Cumulative Effect of Uncertainties on Estimates of Mass Accretion Rates}

The uncertainty in bolometric correction, exclusion of emission lines, and the reradiation of $\mathrm{H}$ Paschen and Balmer continuum emission by the star can only increase the estimated accretion rate, while the uncertainty in binarity and pre-main-sequence tracks can only decrease the estimated accretion rate. When combined, these errors suggest that our accretion rates have a relative uncertainty of $\sim 0.6$ dex and underestimate the accretion rate by a factor of 1.2 (or 2.4, if one assumes no reradiation of the accretion luminosity by the central star).

This error analysis is not a rigorous assessment of the uncertainty in $\dot{M}$ at a specific confidence interval. The uncertainty differs for various targets depending on the accuracy of stellar parameters, and for many targets the error is dominated by uncertainty in $A_{V}$. For 2M 1207-3932, which has an accurate $A_{V}=0$, the $\dot{M}$ is likely underestimated by a factor of 2 (or 4 , if the accretion emission is not reradiated), primarily because we exclude line emission in calculating $\dot{M}$. The standard deviation of $\sim 0.3 \mathrm{dex}$ about this factor of 2 is dominated by systematic uncertainties in the bolometric correction. Systematic uncertainties of this magnitude are also present in all previous UV excess or optical veiling estimates of $\dot{M}$. Several uncertainties, including in the extinction measurements, could be reduced with the addition of near-UV spectra, better photospheric templates across the entire wavelength region, and a better understanding of veiling at long wavelengths.

Intrinsic variability in accretion rate can also affect nonsimultaneous observations. Valenti et al. (2003) describe variability of near-UV emission in repeated IUE spectra of young stars. The average near-UV variability is $29 \%$ for the $15 \mathrm{~K} 1-\mathrm{M} 2$ accretors that were observed at least five times with IUE. The near-UV emission from these stars is dominated by and should correlate directly with the accretion rate (Gullbring et al. 2000). Variability 

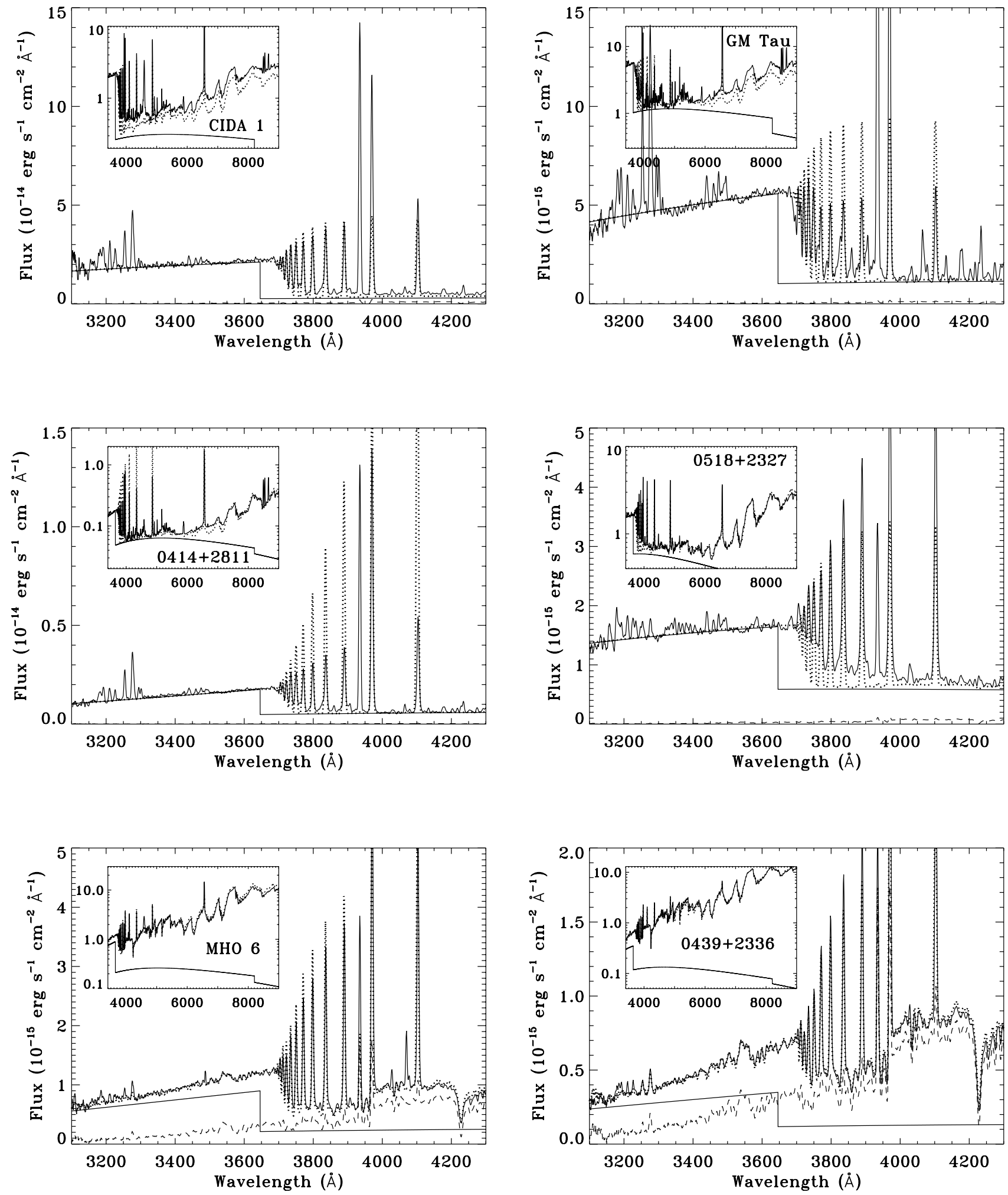

FIg. 8.- Observed emission (solid spectrum), corrected for reddening (Table 1), fitted with the combination of a photospheric template (dashed line; listed in Table 5 and not shown in the inset) and a synthetic accretion spectrum from a slab model (thin solid line; Balmer lines are not shown for clarity). The total fit is shown as a dashed line that overlaps the observed spectrum.

of $0.1-0.2$ dex is also commonly seen in repeated veiling measurements of DF Tau, TW Hya, and AA Tau (Johns-Krull \& Basri 1997; Batalha et al. 2002; Bouvier et al. 2007). Scholz \& Jayawardhana (2006) confirm that variability in accretion rate is also seen for brown dwarfs. We infer that the accretion rate is variable by $\sim 0.2$ dex.

\section{RELATIONSHIPS BETWEEN LINE AND CONTINUUM ACCRETION DIAGNOSTICS}

The Balmer continuum can be difficult to detect because of large extinctions or instrument limitations. Other accretion diagnostics are therefore usually more accessible and can be used 

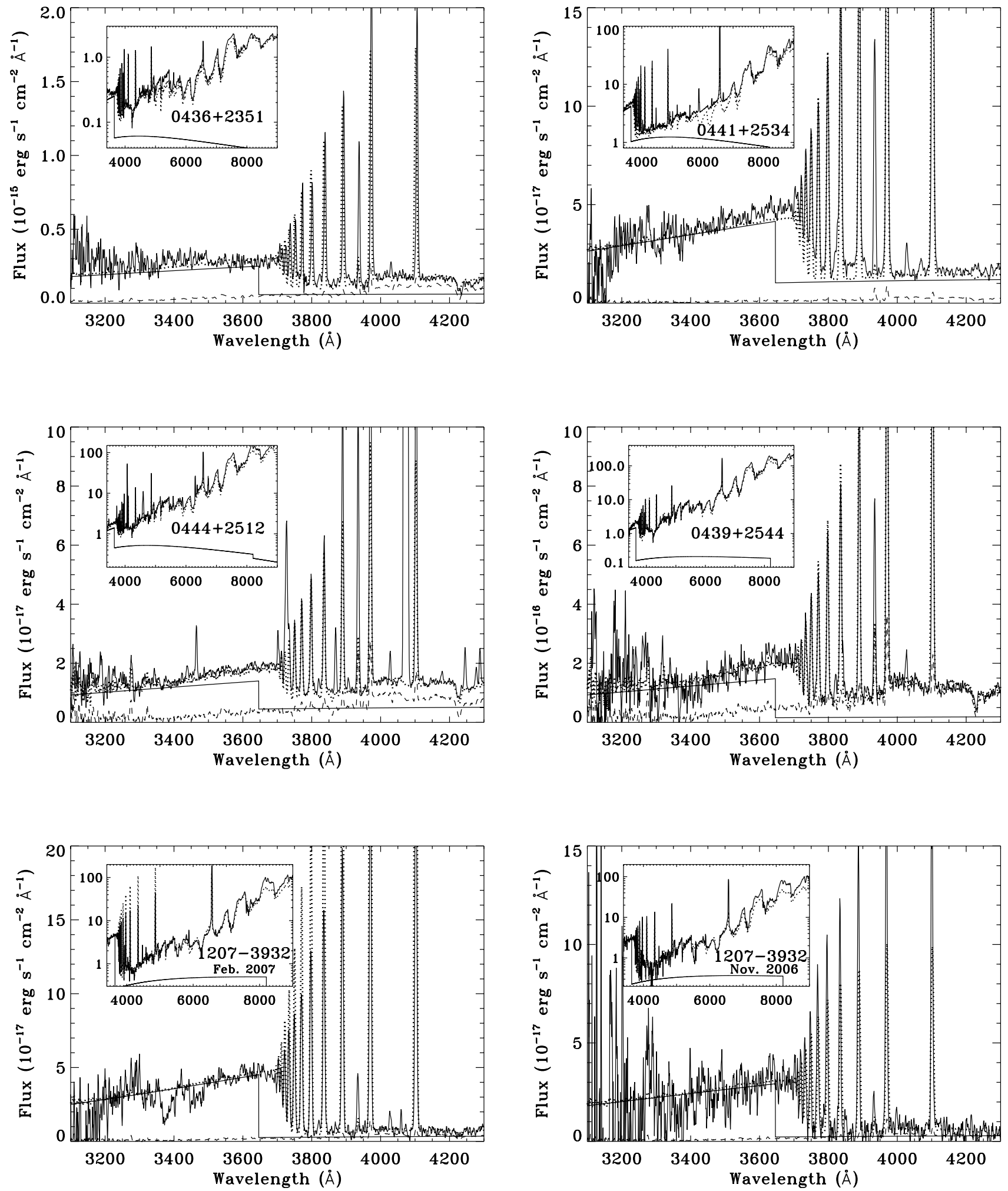

FIG. 8-Continued

to infer accretion rates. In the following subsections we derive and evaluate empirical relationships between emission lines and accretion luminosities and rates. In $\S 5.1$ we calculate accretion rates from published data to incorporate additional objects in our analysis in $\S \S 5.2-5.4$. We then compare the accretion luminosity and resulting accretion rate with $\mathrm{H} \alpha$ line profile measures of accretion ( $§ 5.2)$, line luminosities ( $§ 5.3$ ), and line surface fluxes ( $\S 5.4)$.

\subsection{Calculating Accretion Luminosity from Optical Veiling Measurements}

Although ours are the first Balmer jump measurements of young brown dwarfs, detections of excess Paschen continuum emission have been published. Calculating $\dot{M}$ from these objects increases the overlap of $\dot{M}$ measured using excess continuum 

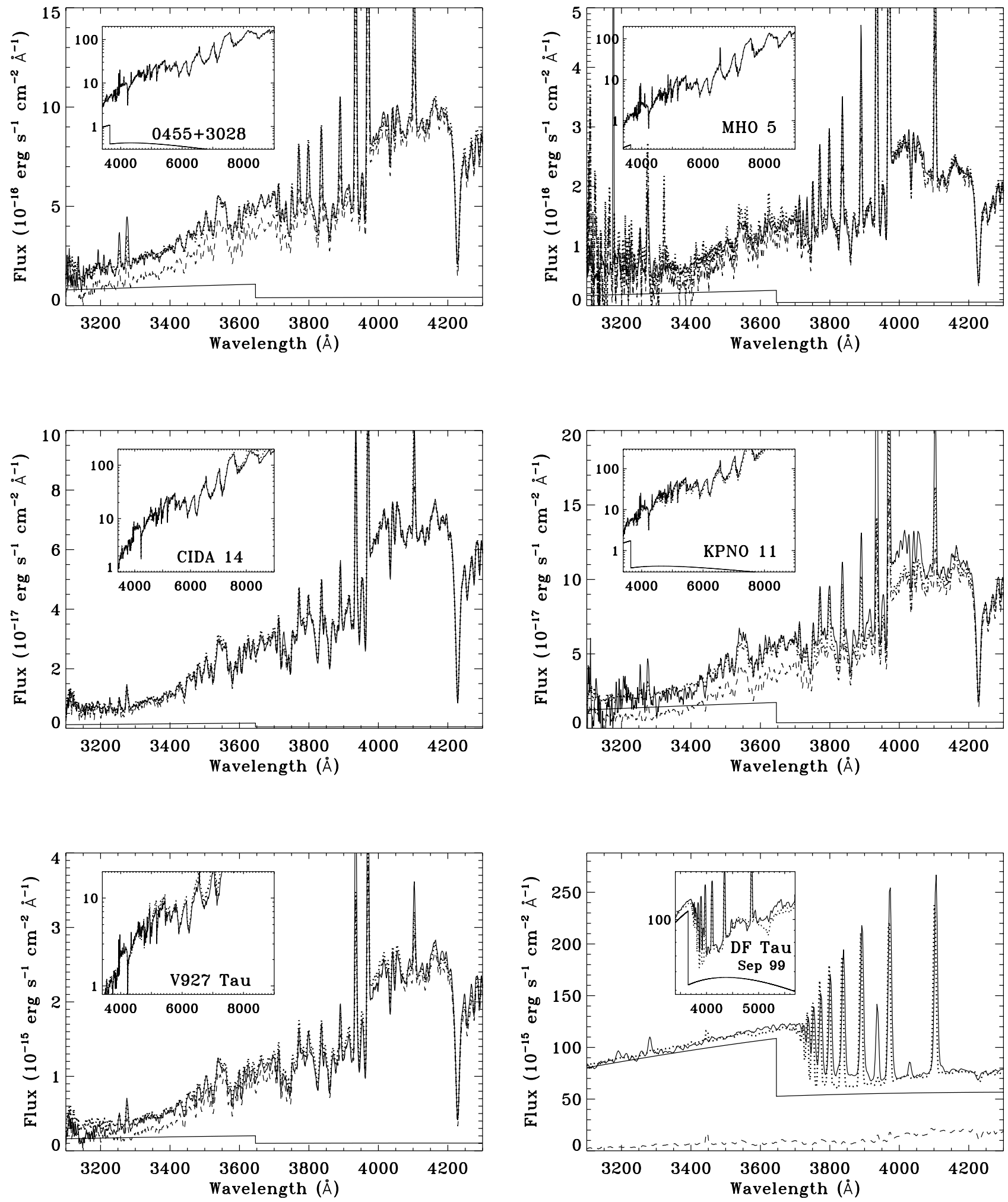

FIG. 8-Continued

emission and $\mathrm{H} \alpha$ line profile modeling (§ 5.2). Kraus et al. (2006) use broadband $H S T$ photometry to measure excess $V$-band emission from several young brown dwarfs. The $V$-band excess is significant when $r_{V}>1.3$, which restricts usage to high mass accretion rates relative to the given stellar mass. We use $\log L_{\text {acc }}=-29.59+$ $\log F_{5450}$ (calculated from Tables 4 and 12) to measure $\dot{M}$ of $10^{-9}$ to $10^{-11} M_{\odot} \mathrm{yr}^{-1}$ for GM Tau, KPNO Tau 4, KPNO Tau 6, and KPNO Tau 12 (Table 14). The $\dot{M}$ from GM Tau based on $V$-band excess is nearly identical to that measured more directly from our low-resolution optical spectra. However, the true $\dot{M}$ was likely $20 \%$ larger during the $H S T$ observation because the $V$-band excess was $\sim 0.2$ mag brighter than was estimated here. 

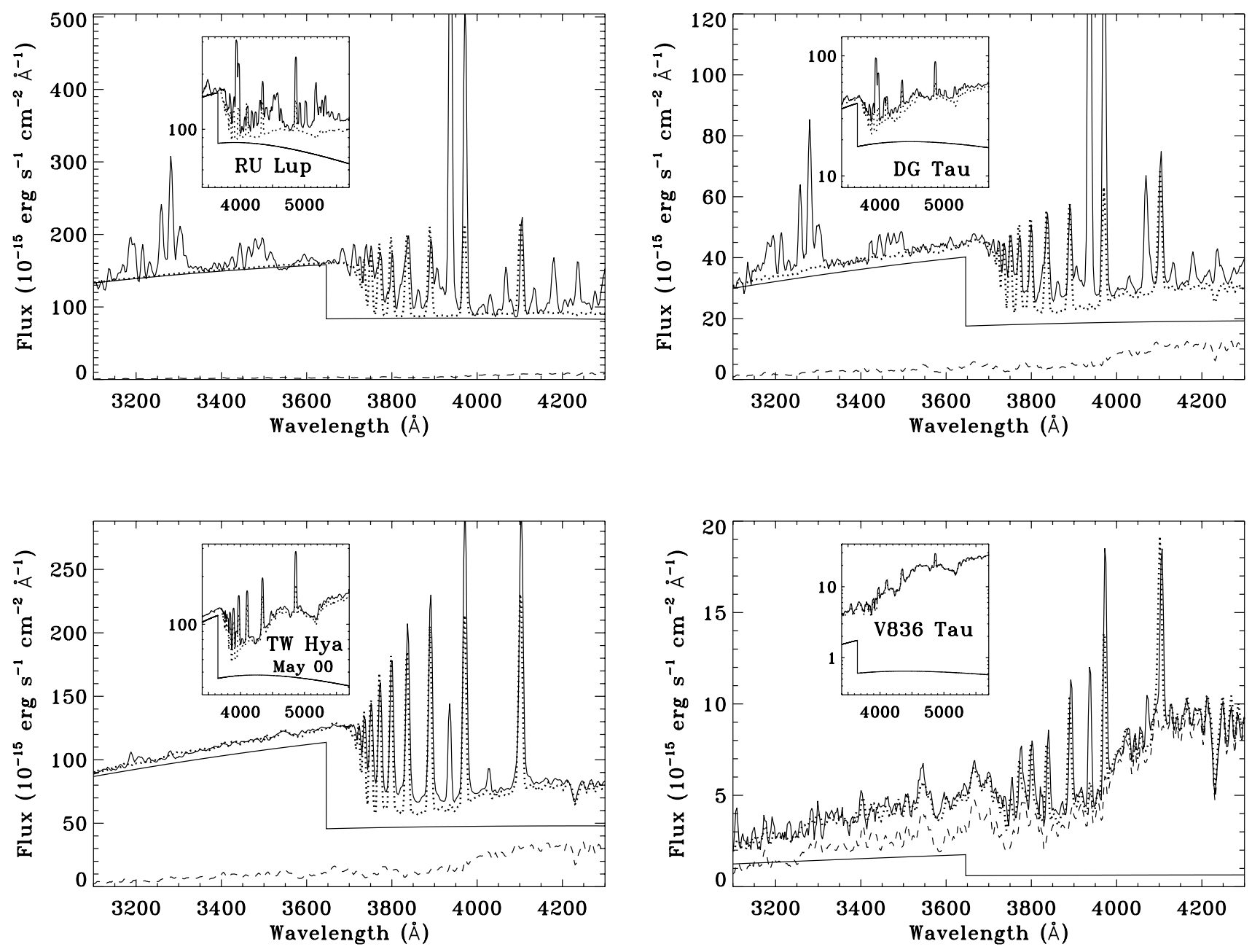

FIG. 8-Continued

Hartigan \& Kenyon (2003) analyzed HST STIS spectra of binaries with early $\mathrm{M}$ spectral types and masses mostly within the $\sim 0.25-0.55 M_{\odot}$ range, including veiling measurements at $6100 \AA$ and luminosities in [O I] $\lambda 6300, \mathrm{H} \alpha$, and $\mathrm{Ca}$ II $\lambda 8542$ lines. Using $\log L_{\mathrm{acc}}=-29.67+\log F_{6190}$ calculated empirically from our Keck I LRIS spectra (see Tables 4 and 12), we find $L_{\text {acc }}$ that are $0.28-0.75$ times those calculated by Hartigan \& Kenyon (2003). We adopt these lower $L_{\text {acc }}$ for further analysis.

\subsection{Comparing UV Excess Accretion Rates to $\mathrm{H} \alpha$ Line Profile Models}

Strong $\mathrm{H} \alpha$ emission is usually attributed to accretion. At high resolution the line profile often includes P Cygni, inverse P Cygni, or self-reversed absorption that corrupts equivalent width measurements (e.g., Reipurth et al. 1996; Muzerolle et al. 2003). Muzerolle et al. $(1998,2001)$ applied magnetospheric

TABLE 13

ERror Estimates

\begin{tabular}{|c|c|c|c|c|}
\hline Parameter & Uncertainty & Dependent Variables & $\log (\sigma \dot{M})^{\mathrm{a}}$ & Systematic \\
\hline 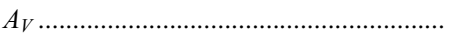 & $0.5 \mathrm{mag}$ & $L_{\text {acc }}$ & 0.4 & $?$ \\
\hline 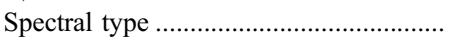 & 0.5 classes & $M, R$ & 0.14 & No? \\
\hline Pre-main-sequence track ........................ & $0.03-0.4$ dex & $M$ & -0.03 to -0.4 & Yes \\
\hline 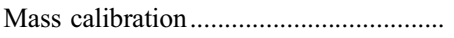 & 0.2 dex & M & 0 to -0.2 & Yes \\
\hline 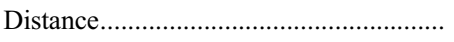 & $15 \mathrm{pc}$ & $L_{\text {acc }}^{2}, R$ & 0.13 & No? \\
\hline 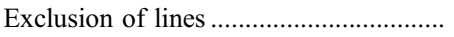 & $0.25 L_{\mathrm{acc}}$ & $L_{\mathrm{acc}}$ & +0.03 to +0.25 & Yes \\
\hline 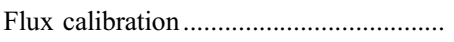 & 0.18 dex & $\propto L_{\mathrm{acc}}, R^{1 / 2}$ & 0.26 & Sometimes \\
\hline 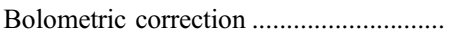 & 0.3 dex & $L_{\mathrm{acc}}$ & +0.3 & Yes \\
\hline Truncation radius & 0.3 dex & $R_{\text {in }}$ & 0.08 & $?$ \\
\hline Photospheric template .............................. & $0-0.3$ dex & $L_{\text {acc }}$ & 0 to +0.3 & No? \\
\hline 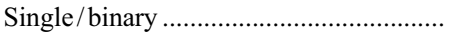 & 0.15 dex & $R$ & -0.3 & Yes \\
\hline 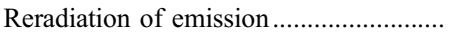 & 0.3 dex & $L_{\text {acc }}$ & +0.3 & Yes \\
\hline
\end{tabular}

${ }^{\text {a }}$ Plus or minus indicates that the uncertainty is only in one direction. 
TABLE 14

Estimated $\dot{M}$ From $V$-Band Excess

\begin{tabular}{|c|c|c|c|c|c|c|c|c|}
\hline Star & $\begin{array}{c}V \\
(\mathrm{mag})\end{array}$ & $\begin{array}{c}M \\
\left(M_{\odot}\right)\end{array}$ & $R$ & $r_{V}$ & $\begin{array}{c}A_{V} \\
(\mathrm{mag})\end{array}$ & $\begin{array}{c}f_{5500} \\
\left(10^{-16} \mathrm{ergs} \mathrm{cm}^{-2} \mathrm{~s}^{-1} \AA^{-1}\right)\end{array}$ & $\begin{array}{c}L_{\mathrm{acc}} \\
\left(10^{-4} L_{\odot}\right)\end{array}$ & $\begin{array}{c}\dot{M} \\
\left(10^{-10} M_{\odot} \mathrm{yr}^{-1}\right)\end{array}$ \\
\hline GM Tau & 17.577 & 0.15 & 0.93 & $1.7 \pm 0.9$ & 2.0 & 13 & 79 & 19 \\
\hline KPNO 4 & 24.722 & 0.01 & 0.42 & $1.6 \pm 0.8$ & 4.0 & 0.11 & 0.67 & 1.1 \\
\hline KPNO 6 & 22.292 & 0.02 & 0.68 & $1.1_{-0.7}^{+0.9}$ & 0.7 & 0.044 & 0.27 & 0.36 \\
\hline
\end{tabular}

NoтE.-Magnitudes, excess, and stellar properties (except for GM Tau) from Kraus et al. (2006).

accretion models to calculate the formation and radiative transfer of $\mathrm{H} \alpha$ lines. These models treat the line profile, scaled by stellar parameters, as a proxy for $\dot{M}$. Muzerolle et al. $(2003,2005)$ applied these models to very low mass stars and brown dwarfs to estimate accretion rates. The left panel of Figure 9 and Table 15 compare mass accretion rates for stars with $\dot{M}$ measured from both $\mathrm{H} \alpha$ line profile models and UV excess measurements. The UV excess accretion rate measurements are $\sim 0.84$ dex larger than the $\mathrm{H} \alpha$ accretion rate measurements with a standard deviation of $0.3 \mathrm{dex}$, if MHO 5 and CIDA 14 are ignored. If both the $\mathrm{H} \alpha$ emission and weak excess Balmer emission from those two stars are attributable to accretion, then the UV excess measures are 0.6 dex larger than the $\mathrm{H} \alpha$ accretion measures but with a larger (0.5 dex) source-to-source variation.

This difference is consistent with the systematic methodological differences between the two methods. However, that the systematic errors are in opposite directions for the two methods is unlikely. For the UV excess method several uncertainties (see $\S$ 4.3) would serve to increase our $\dot{M}$ estimates and therefore the discrepancy between the two methods. The uncertainty in the $\mathrm{H} \alpha \dot{M}$ calculations of $\sim 3-5$ is dominated by uncertainty in the disk truncation radius of the inner disk, assumed to be $2.2 R_{*}-3 R_{*}$ (Muzerolle et al. 2003). The model $\mathrm{H} \alpha$ line width is proportional to $M_{*}^{0.5}$, so overestimating $M_{*}$ will lead to underestimating $\dot{M}$ for both methods. Intrinsic variability in $\dot{M}$ can explain much of the scatter once the systematic variation between these two methods is corrected.

In the Muzerolle et al. $\mathrm{H} \alpha$ line profile models, the accretion rate is estimated from the line profile along with the stellar mass and disk inclination. Although $\dot{M}$ is correlated with the $\mathrm{H} \alpha$ line width in the models, different $\dot{M}$ may vary by more than an order of magnitude for equivalent $10 \%$ widths (see also Kurosawa et al. 2006). Natta et al. (2004) found an empirical relationship between
$\dot{M}$ and $\mathrm{H} \alpha 10 \%$ width, which is used in the absence of $\mathrm{H} \alpha$ line profile modeling. We find a 0.86 dex scatter between the $\dot{M}$ measured here from UV excess and $\dot{M}$ from the Natta et al. (2004) $\mathrm{H} \alpha$ relationship (Fig. 9, right panel) and a similar amount of scatter for a similar best-fit line ${ }^{4}$ from Table 15.2M 1207-3932 is a particular outlier on this plot, with an $\dot{M}$ estimated from the $\mathrm{H} \alpha$ $10 \%$ width that is 2 orders of magnitude larger than that measured from the UV excess.

\subsection{Empirical Relationships between Line and Continuum Luminosities}

In $\S 3.4$ we qualitatively describe that excess continuum and line emission are produced by accretion processes. Lines from accretors exhibit a wide range of profiles in high-resolution spectra, with equivalent widths that are correlated with veiling (e.g., Hamann \& Persson 1992; Batalha et al. 1996; Muzerolle et al. 1998; Beristain et al. 2001; Mohanty et al. 2005). Figure 10 shows that the luminosity in many lines $\left(L_{\text {line }}\right)$ is tightly correlated with accretion continuum luminosity, although this relationship does not require that the emission lines and continuum are produced by the same gas. We improve the fits for the $\mathrm{H} \alpha$, [O I ] $\lambda 6300$, and Ca II $\lambda 8542$ lines by supplementing our data with results from Hartigan \& Kenyon (2003). All line and accretion luminosities used in this subsection were measured from simultaneous data.

Linear fits of the relationship between $\log L_{\text {acc }}$ and $\log L_{\text {line }}$ are calculated using the statistical package ASURV (Feigelson \& Nelson 1985), with upper limits ignored (see Table 16 and Fig. 10). Most upper limits are not significant and their inclusion does

4 We find that $\dot{M}=-14.0+0.014$ (H $\alpha 10 \%$ width) from the data in Table 15 , excluding upper limits, and $\dot{M}=-12.6+0.010$ ( $\mathrm{H} \alpha 10 \%$ width) if we combine these data with additional $\dot{M}$ calculated from the Ca II $\lambda 8662$ line fluxes from Mohanty et al. (2005; see also $\S 5.4)$.

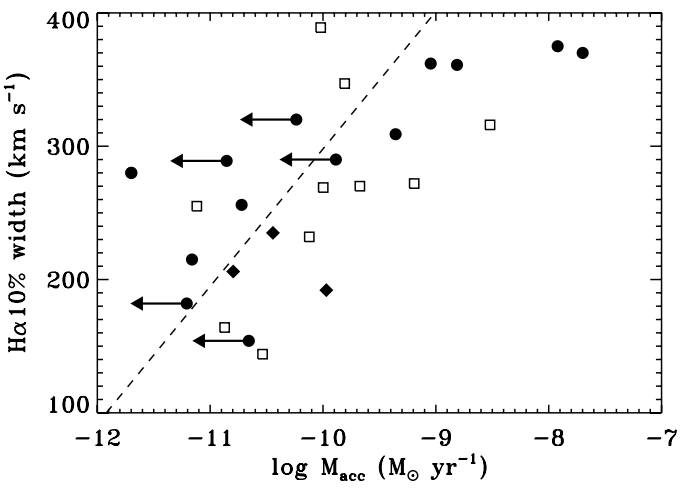

FIG. 9.-UV excess measure of $\dot{M}$ vs. nonsimultaneous measurements of $\dot{M}$ from models of the H $\alpha$ line profiles (left; from Muzerolle et al. 2000, 2003, 2005) and vs. nonsimultaneous measurements of the $\mathrm{H} \alpha 10 \%$ width (right). The circles are accretors with $\dot{M}$ measured from UV excess, and the diamonds are accretors with $\dot{M}$ measured

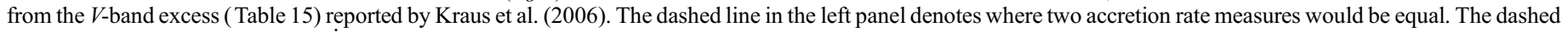
line in the right panel denotes the $\dot{M}-\mathrm{H} \alpha 10 \%$ width relationship calculated by Natta et al. (2004). 
TABLE 15

Comparing $\dot{M}$ Measured from UV Excess and $\mathrm{H} \alpha$

\begin{tabular}{|c|c|c|c|c|c|c|}
\hline \multirow[b]{2}{*}{$\mathrm{S}_{\mathrm{TAR}}$} & \multicolumn{3}{|c|}{ H $\alpha$ Profile $^{\mathrm{a}}$} & \multicolumn{2}{|c|}{ UV EXCESS ${ }^{\mathrm{b}}$} & \multirow[b]{2}{*}{ REFERENCES } \\
\hline & $\begin{array}{c}\mathrm{H} \alpha 10 \% \text { Width } \\
\left(\mathrm{km} \mathrm{s}^{-1}\right)\end{array}$ & $\begin{array}{c}\mathrm{H} \alpha \mathrm{EW} \\
(\AA)\end{array}$ & $\log \left(\dot{M} / M_{\odot} \mathrm{yr}^{-1}\right)$ & $\begin{array}{c}\mathrm{H} \alpha \mathrm{EW} \\
(\AA)\end{array}$ & $\log \left(\dot{M} / M_{\odot} \mathrm{yr}^{-1}\right)^{\mathrm{c}}$ & \\
\hline MHO $5 \ldots \ldots \ldots \ldots \ldots \ldots \ldots \ldots \ldots \ldots$ & 154 & -60 & -10.8 & -43 & -10.7 & 1 \\
\hline MHO 6 & 309 & -25 & -10.3 & -55 & -9.3 & 1 \\
\hline 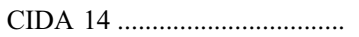 & 289 & -34 & -10.3 & -10 & -10.9 & 1 \\
\hline 2M 0414+2811 ....................... & 362 & -250 & -10 & -288 & -8.9 & 2 \\
\hline 2M 0439+2544 & 215 & -102 & -11.3 & -161 & -11.1 & 2 \\
\hline 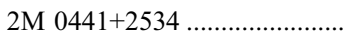 & 256 & -234 & -11.3 & -600 & -10.5 & 2 \\
\hline 2M $0455+3028 \ldots \ldots \ldots \ldots \ldots \ldots \ldots$ & 320 & -24.5 & $\ldots$ & -9.5 & $<-10.5$ & 2 \\
\hline 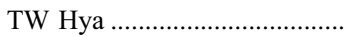 & 361 & $\ldots$ & -9.3 & $\ldots$ & -8.7 & 3 \\
\hline 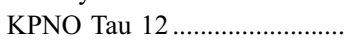 & 206 & -66.8 & -11.4 & $\ldots$ & $-10.8^{\mathrm{d}}$ & 2 \\
\hline 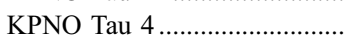 & 192 & -38.4 & $<-12$ & $\ldots$ & $-10.0^{\mathrm{d}, \mathrm{e}}$ & 2 \\
\hline 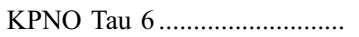 & 235 & -41.1 & -11.4 & $\ldots$ & $-11.0^{\mathrm{d}}$ & 2 \\
\hline CIDA $1 \ldots \ldots \ldots \ldots \ldots \ldots \ldots \ldots \ldots$ & 375 & 126 & $\ldots$ & 232 & -7.9 & 4 \\
\hline 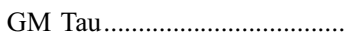 & 370 & 113 & $\ldots$ & 281 & -8.7 & 4 \\
\hline 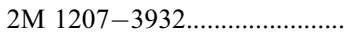 & 280 & $\ldots$ & $\ldots$ & 200,400 & -11.7 & 5,6 \\
\hline KPNO $11 \ldots \ldots \ldots \ldots \ldots \ldots \ldots \ldots \ldots$ & 182 & 17.7 & $\ldots$ & 13.6 & $<-11.2$ & 5 \\
\hline 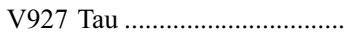 & 290 & 7.0 & $\ldots$ & 10.7 & $<-9.9$ & 1 \\
\hline 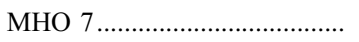 & 116 & 9.0 & $\ldots$ & 7.4 & $\ldots$ & 1 \\
\hline $\begin{array}{l}\text { a } \text { Measured during } \mathrm{H} \alpha \dot{M} \mathrm{~m} \\
\mathrm{~b} \text { Measured during UV exces } \\
\mathrm{c} \text { May differ from Table } 10 ; \\
\mathrm{d} \text { Calculated from } V \text {-band ex } \\
\text { e } M \text { and } R \text { from Table } 14 .\end{array}$ & $\begin{array}{l}\text { Irement. } \\
\text { measurement. } \\
\text { and } R_{*} \text { adopted her } \\
\text {; see } \S 5.1 \text {. }\end{array}$ & rom Muzer & et al. & & & \\
\hline $\begin{array}{l}\text { REFERENCES.-(1) Muzerolle } \\
005 \text {; (6) Schaefer et al. } 2006 .\end{array}$ & 1. 2003; (2) Muze & le et al. 20 & (3) Muzerolle et & $000 ;(4) \mathrm{V}$ & e \& Basri 2003; & ohanty \\
\hline
\end{tabular}

not substantially change the results. 2M 0455+3028, KPNO 11, CIDA 14, MHO 5, and V927 Tau (Fig. 10, squares) are not included in these fits because the source of the excess Balmer emission is uncertain. For several lines at $>5600 \AA$, the high $\dot{M}$ range for these fits is dominated by CIDA 1 and GM Tau, both of which have large uncertainty in $A_{V}$ and therefore $\dot{M}$. The fits based on few data points should be used with caution.

The $\mathrm{H} \alpha-L_{\mathrm{acc}}$ correlation has more scatter than the correlation between higher Balmer lines and $L_{\text {acc }}$ because the larger oscillator strength of $\mathrm{H} \alpha$ can lead to stronger wind absorption. The Balmer and $\mathrm{Ca}$ II $\mathrm{H}$ and $\mathrm{K}$ line luminosities from KPNO 11, $2 \mathrm{M} 0455+3028$, MHO 5, and CIDA 14 lie above the linear fit to

TABLE 16

Line Luminosity-Accretion Luminosity Relationships for Selected Lines

\begin{tabular}{|c|c|c|c|c|c|}
\hline Diagnostic & $\begin{array}{c}\lambda \\
(\AA)\end{array}$ & $a^{\mathrm{a}}$ & $b^{\mathrm{a}}$ & $N^{\mathrm{b}}$ & $\sigma^{\mathrm{c}}$ \\
\hline He I................................ & 5016 & $3.3 \pm 0.3$ & $1.04 \pm 0.05$ & 18 & 0.30 \\
\hline Не $\mathrm{I}$ & 5876 & $5.3 \pm 0.7$ & $1.46 \pm 0.12^{\mathrm{d}}$ & 12 & 0.34 \\
\hline 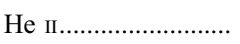 & 4686 & $3.7 \pm 0.5$ & $1.04 \pm 0.08$ & 16 & 0.38 \\
\hline 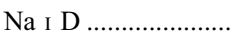 & 5893 & $3.3 \pm 0.7$ & $1.09 \pm 0.11$ & 11 & 0.41 \\
\hline 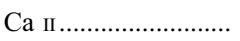 & 8542 & $2.5 \pm 0.5$ & $1.02 \pm 0.11$ & 20 & 0.44 \\
\hline 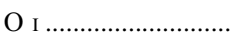 & 6300 & $2.8 \pm 0.8$ & $0.96 \pm 0.16$ & 26 & 0.67 \\
\hline $\mathrm{H} \alpha$ & 6563 & $2.0 \pm 0.4$ & $1.20 \pm 0.11$ & 35 & 0.57 \\
\hline $\mathrm{H} \beta$ & 4861 & $2.6 \pm 0.2$ & $1.22 \pm 0.05$ & 19 & 0.26 \\
\hline $\mathrm{H} \gamma$ & 4340 & $3.0 \pm 0.2$ & $1.24 \pm 0.04$ & 19 & 0.21 \\
\hline $\mathrm{Ca}$ II $\mathrm{K}$ & 3932 & $2.0 \pm 0.3$ & $0.96 \pm 0.05$ & 19 & 0.33 \\
\hline H9 ....................... & 3771 & $3.4 \pm 0.2$ & $1.17 \pm 0.03$ & 19 & 0.18 \\
\hline
\end{tabular}

\footnotetext{
${ }^{\text {a }}$ Here $\log L_{\text {line }}=a+b \log L_{\text {acc }}$, with $L_{\text {acc }}$ and $L_{\text {line }}$ in units of $L_{\odot}$.

b Number of accretors with detections.

${ }^{c}$ Standard deviation of detected line fluxes from linear fit.

d Significant deviation from 1.0; use with caution outside our $\dot{M}$ range.
}

accretion luminosity. Since these four stars are tentative weak accretors, such line emission is likely produced by the chromosphere. The relationship between the Balmer and $\mathrm{Ca}$ II $\mathrm{K}$ lines and the accretion luminosity derived from our data is consistent with the results from Gullbring et al. (1998). The luminosities in these lines are systematically $\sim 0.5$ dex lower in the Valenti et al. (1993) sample than in the Gullbring et al. (1998) sample. ${ }^{5}$ The nature of this discrepancy is not known.

A large amount of scatter in the relationship between [O I] line luminosity and accretion luminosity arises because the [O I] emission line forms in an accretion-powered outflow (Hamann 1994; Hartigan et al. 1995). The comparison of the Hartigan \& Kenyon (2003) sample and our sample may be corrupted because $[\mathrm{O}$ I] emission is typically spatially extended (e.g., Hartigan et al. 2004). Any extended [O I] emission could lie outside the narrow $0.2^{\prime \prime}$ slit width used by Hartigan et al. (1995), but entirely within our larger $1.0^{\prime \prime}$ slit. The outflow line luminosities are correlated with accretion rate, although strong accretors sometimes drive weak outflows while some weak accretors may drive powerful outflows. For example, the outflow lines from GM Tau and 2M $0414+2811$ are substantially stronger than those from CIDA 1 , despite the higher mass accretion rate onto CIDA 1. Similarly, many bright outflow lines are detected from $2 \mathrm{M} 0444+2512$ despite a modest mass accretion rate. The $\mathrm{He}$ I and $\mathrm{Na}$ I $\mathrm{D}$ lines are usually associated with both infall and outflow (Hartigan et al. 1995; Beristain et al. 2001; Edwards et al. 2006) but here are more tightly correlated with the accretion luminosity than [O I].

\footnotetext{
${ }^{5}$ In Valenti et al. (1993) and here, the Balmer and $\mathrm{Ca}$ II $\mathrm{H}$ and $\mathrm{K}$ lines are measured directly from the spectrum while Gullbring et al. (1998) measure line fluxes after the subtraction of a photospheric template. These two different methods would slightly exacerbate the discrepancy between the line luminosities.
} 

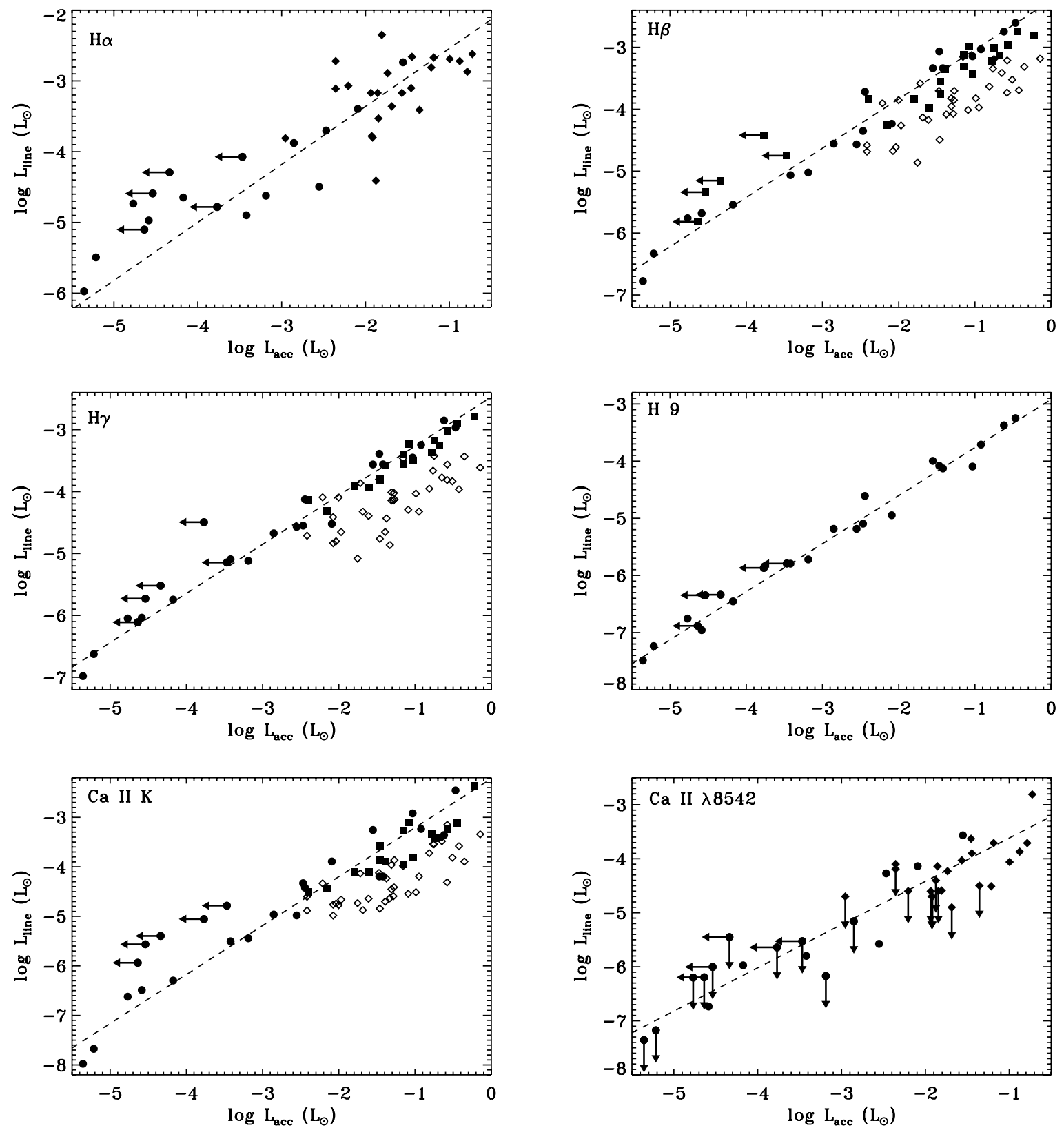

FIG. 10.- Relationship between selected line luminosities and the accretion continuum luminosity. The filled circles represent the accretors with strong excess Balmer continuum emission that is produced by accretion. The upper limits in $L_{\text {acc }}$ are the five possible accretors, which have weak Balmer continuum emission that may be attributed to either accretion or chromospheric activity. We include results from Valenti et al. (1993; open diamonds; corrected for the $140 \mathrm{pc}$ distance to Taurus) and Gullbring et al. (1998; filled squares) for fits to the $\mathrm{H} \beta, \mathrm{H} \gamma$, and $\mathrm{Ca}$ II $\mathrm{K}$ line luminosities and from Hartigan \& Kenyon (2003; filled triangles) for fits to the $\mathrm{H} \alpha$, Ca II 28542 , and $\left[\mathrm{O}_{\mathrm{I}}\right]$ line luminosities. The dashed lines are linear fits to the data as described in $\S 5.3$ and Table 16.

\subsection{Comparison with $\dot{M}$ Measurements from Emission-Line Fluxes}

In $\S 5.3$ we derive empirical relationships between line and accretion continuum luminosity. Lacking flux-calibrated spectra, Muzerolle et al. (1998) and Mohanty et al. (2005) converted the $\mathrm{Ca}$ II equivalent width into line flux per stellar surface area and then correlated the line flux directly with $\dot{M}$. Such a correlation naturally corrects for radius uncertainties introduced by errors in extinction or distance. Compared with equation (1), correlating $L_{\text {line }} / R_{*}^{2}$ with $\dot{M}$ will be in error in $\dot{M}$ by a factor of $M / R^{3}$.

Figure 11 compares the $\mathrm{Ca}$ II 28542 , Ca II 28662 , and He I 25876 line fluxes with accretion rate for the low-mass accretors presented here, high-mass accretors from Muzerolle et al. (1998; for the 28542 line), and low- and high-mass accretors from
Mohanty et al. (2005; for the $\lambda 8662$ line). ${ }^{6}$ Those data have $\dot{M}$ and $\mathrm{Ca}$ II line fluxes measured from noncontemporaneous observations and will suffer from variability. The $F$ (Ca II 28542)$\dot{M}$ and $F(\mathrm{He}$ I 25876$)-\dot{M}$ relationships are shallower than was previously measured from only the high-mass sample, while the $F(\mathrm{Ca}$ II 28662$)-\dot{M}$ relationship is similar to that derived by Mohanty et al. (2005). The standard deviations between $\dot{M}$

${ }^{6}$ We calculate $\log \dot{M}=1.28 \log F(\mathrm{Ca}$ II 28542$)-16.6, \quad \log \dot{M}=$ $1.03 \log F(\mathrm{Ca}$ II 28662$)-15.2$, and $\log \dot{M}=1.63 \log F($ He I 25876$)-17.9$ from line fluxes and UV excess measures of accretion. We also note that the relative fluxes in the three $\mathrm{Ca}$ II IR triplet lines are usually consistent with the optically thick limit of 1:1:1 (Herbig \& Soderblom 1980; Hamann \& Persson 1989), whether the emission is in a broad or narrow line (Batalha et al. 1996). Even at high resolution all three $\mathrm{Ca}$ II IR triplet lines blend with Paschen lines. 

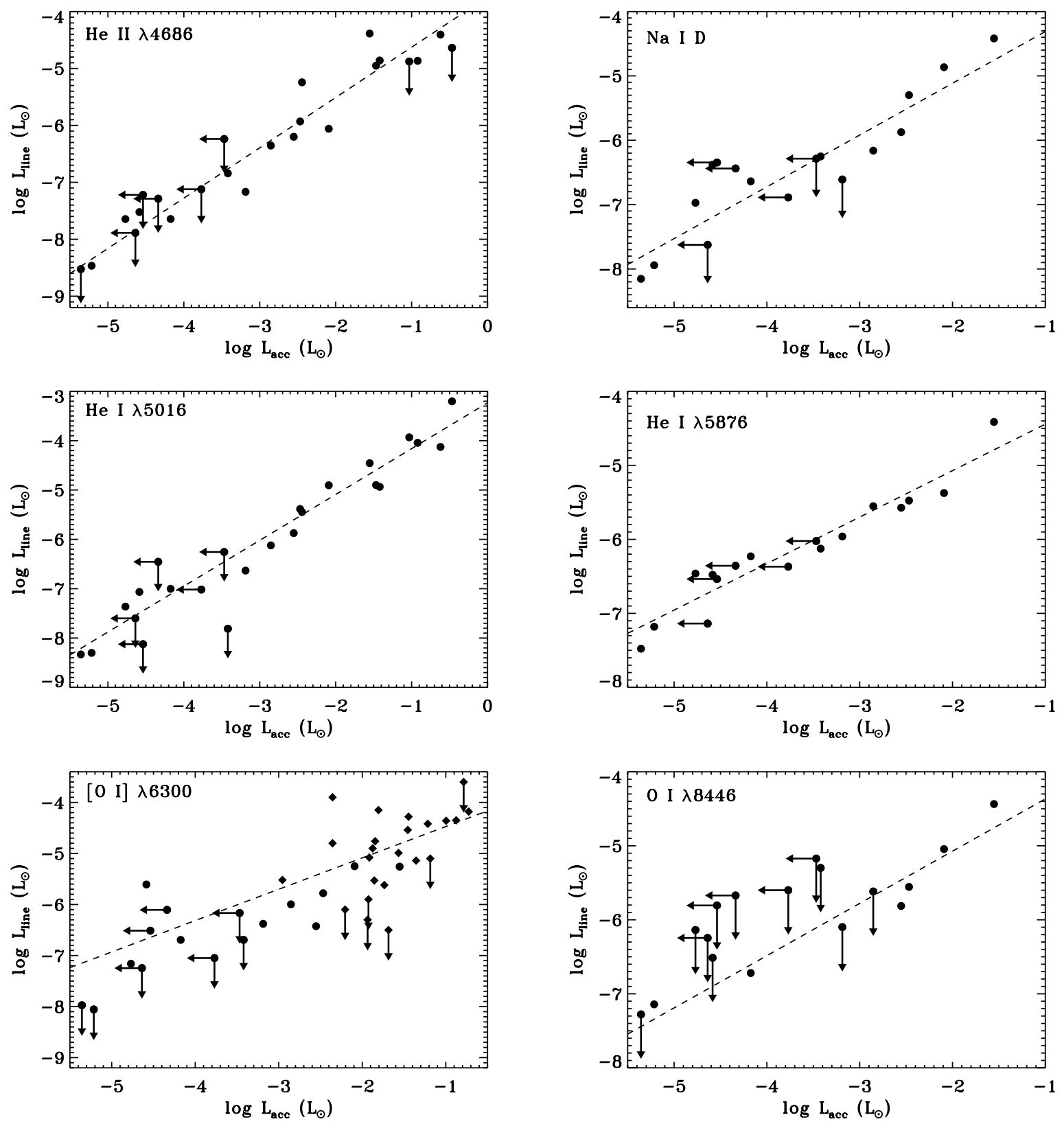

FIG. 10-Continued

predicted from these line correlations and the $\dot{M}$ measured from UV continuum excess are 1.0, 0.71, and 1.1 dex for Ca II $\lambda 8542$, $\mathrm{Ca}$ II $\lambda 8662$, and He I $\lambda 5876$ line fluxes, respectively.

\section{DISCUSSION}

\subsection{Selecting Methods to Measure $\dot{M}$}

In the magnetospheric accretion paradigm, the Paschen continuum emission is produced in optically thick postshock gas, the Balmer continuum emission is produced in optically thin preshock gas, and the Balmer line emission is mostly produced primarily in the magnetospheric accretion column (Hartmann et al. 1994; Calvet \& Gullbring 1998; Muzerolle et al. 2001; Kurosawa et al. 2006). In the simplistic plane-parallel models all three accretion diagnostics are produced by the same gas. In either case, the accretion energy is reprocessed into Balmer and Paschen continuum emission, which can be measured and subsequently con- verted into accretion rate. Previously, the accretion continuum measurements were applied only to accretors with $\sim 0.3-2 M_{\odot}$. Muzerolle et al. (2003) developed models to measure $\dot{M}$ from $\mathrm{H} \alpha$ line profiles for only the very low mass stars and brown dwarfs. At higher mass accretion rates the $\mathrm{H} \alpha$ emission line profile is difficult to use for estimating $\dot{M}$ because emission and absorption in strong stellar outflows corrupt the line profile (Muzerolle et al. 2001; Kurosawa et al. 2006). We provide the first measurements of the Balmer continuum emission from very low mass stars and brown dwarfs and describe this emission as qualitatively similar to that from higher mass accretors. The UV excess measures of $\dot{M}$ are $\sim 4-7$ times larger than those from $\mathrm{H} \alpha$ line profile modeling with some source-to-source differences that may be methodological or related to intrinsic source variability.

In the context of magnetospheric accretion models, that the excess accretion continuum and the $\mathrm{H} \alpha$ emission yield different $\dot{M}$ may not be surprising because they form in different locations. 

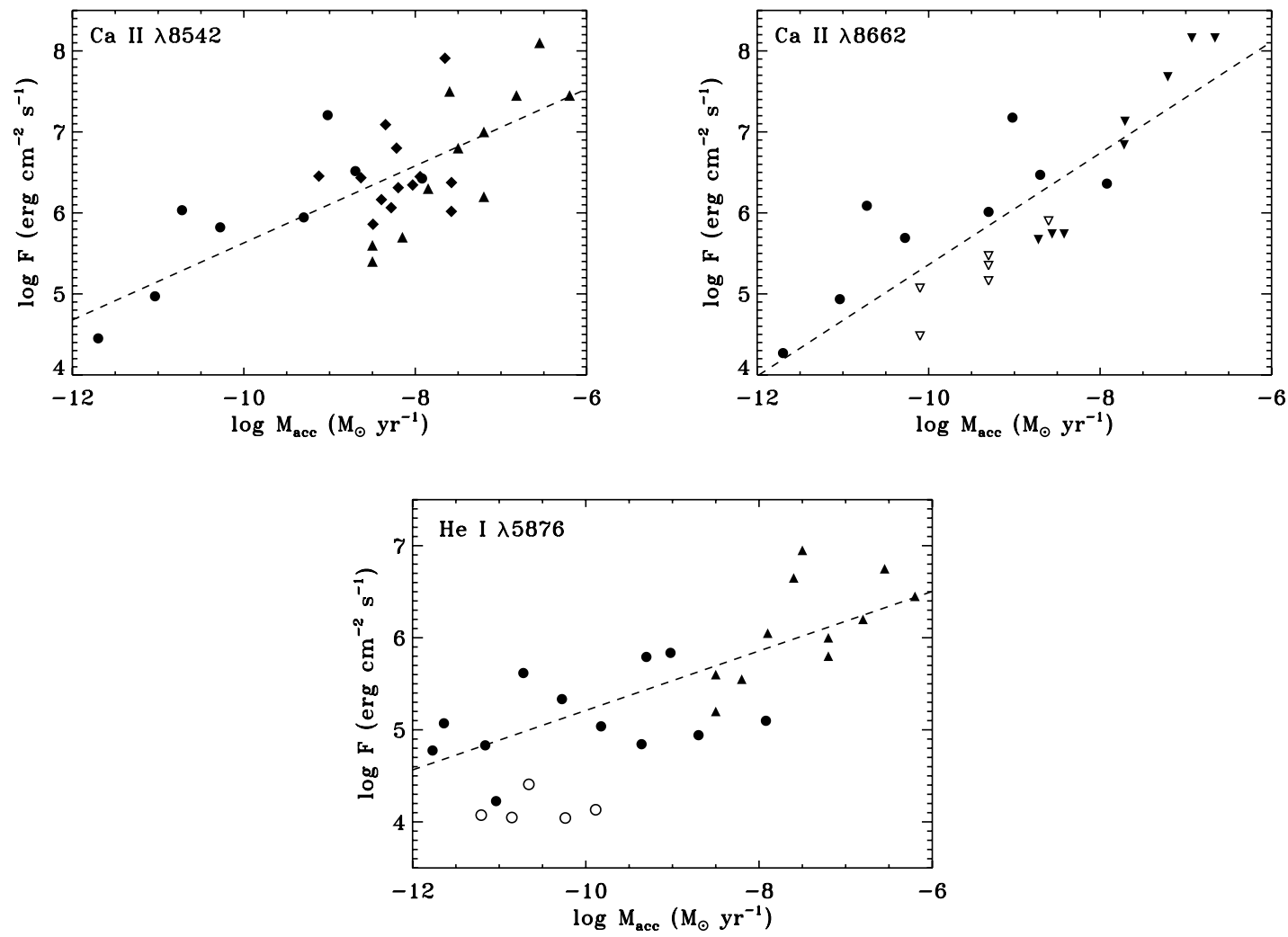

FIG. 11.-Ca II 28542 (top left) and Ca II $\lambda 8662$ (top right) line flux vs. mass accretion rate. We compare our data (filled circles for accretors, open circles for the five possible accretors) with those from Hartigan \& Kenyon (2003; filled diamonds for Ca II 28542 ), Muzerolle et al. (1998; filled triangles for Ca II 28542 and He I 25876 ), and Mohanty et al. (2005; downward-pointing triangles for $\mathrm{Ca}$ II 28662 ). The open downward-pointing triangles in the top right panel signify that the $\dot{M}$ was measured by $\mathrm{H} \alpha$ modeling and is multiplied by 5 . The dashed lines in both panels show the best fit through the data.

The primary advantage of the $\mathrm{H} \alpha$ models is that the line profile is independent of the intrinsic line luminosity and only moderately dependent on the stellar mass. According to Muzerolle et al. (2003), the uncertainty in $\dot{M}$ from $\mathrm{H} \alpha$ modeling is a factor of 3-5 and is dominated by uncertainty in the size of the magnetosphere. The factor of $\sim 4$ random error in UV excess measures of accretion is dominated by errors in extinction and distance. The UV excess method relies predominantly on directly observable parameters, with modeling required only for calculating a moderate bolometric correction to derive $L_{\text {acc. }}$. The accretion energy is converted to $\dot{M}$ by equation (1). In contrast, accretion rates from the $\mathrm{H} \alpha$ line profile rely on a correct interpretation and modeling of the line profile in the magnetospheric funnel flow and may be complicated by outflows, emission from hot spots, complex magnetic fields, or unexpected geometries.

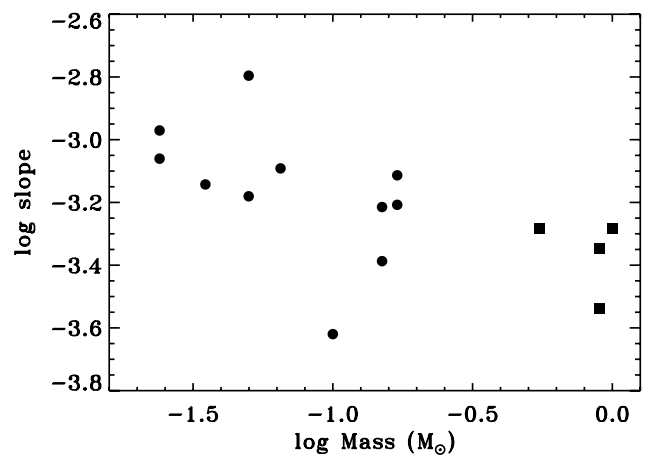

Figures 12 and 13 compare the spectral properties of the excess hydrogen continuum emission versus stellar mass and mass accretion rate. The size of the Balmer jump tends to increase with decreasing $M$ (and $\dot{M})$, consistent with lower $n_{e}\left(\sim 3 \times 10^{13} \mathrm{~cm}^{-3}\right.$ for 2M 1207-3932 compared with $\sim 2 \times 10^{14} \mathrm{~cm}^{-3}$ for higher mass accretors) in single-temperature slab models or with a larger contribution of emission from the optically thin preshock gas relative to optically thick postshock gas in the magnetospheric accretion models. The slope of the Balmer continuum, which may be affected by our atmospheric correction, tends to increase with decreasing $M($ and $\dot{M})$. This is consistent with a lower temperature ( $\sim 7500 \mathrm{~K}$ for $2 \mathrm{M} 1207-3932$, compared with $\sim 8000-12,000 \mathrm{~K}$ for higher mass accretors) in the plane-parallel slab models. Muzerolle et al. (2003) describe that the temperature in the magnetospheric accretion flow must be at least $10^{4} \mathrm{~K}$ to have sufficient

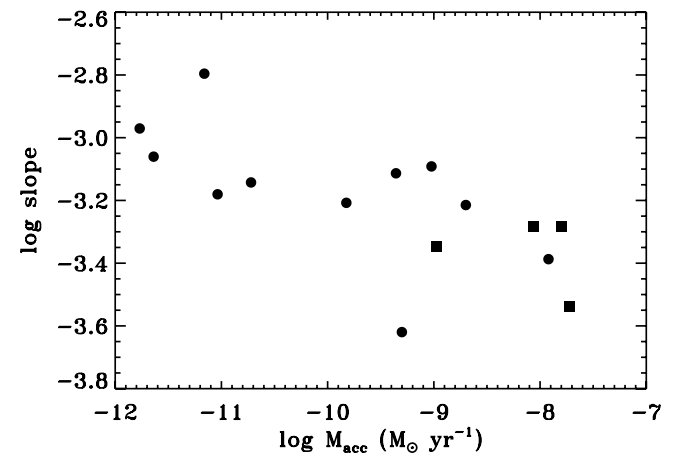

Fig. 12.- Slope of the Balmer continuum vs. stellar mass $(l e f t)$ and $\dot{M}(r i g h t)$. The circles are from our Keck I LRIS data, the squares from HST STIS G430L spectra. The slope of the Balmer continuum increases to smaller $M$ and $\dot{M}$, suggesting that it is produced at cooler gas. 

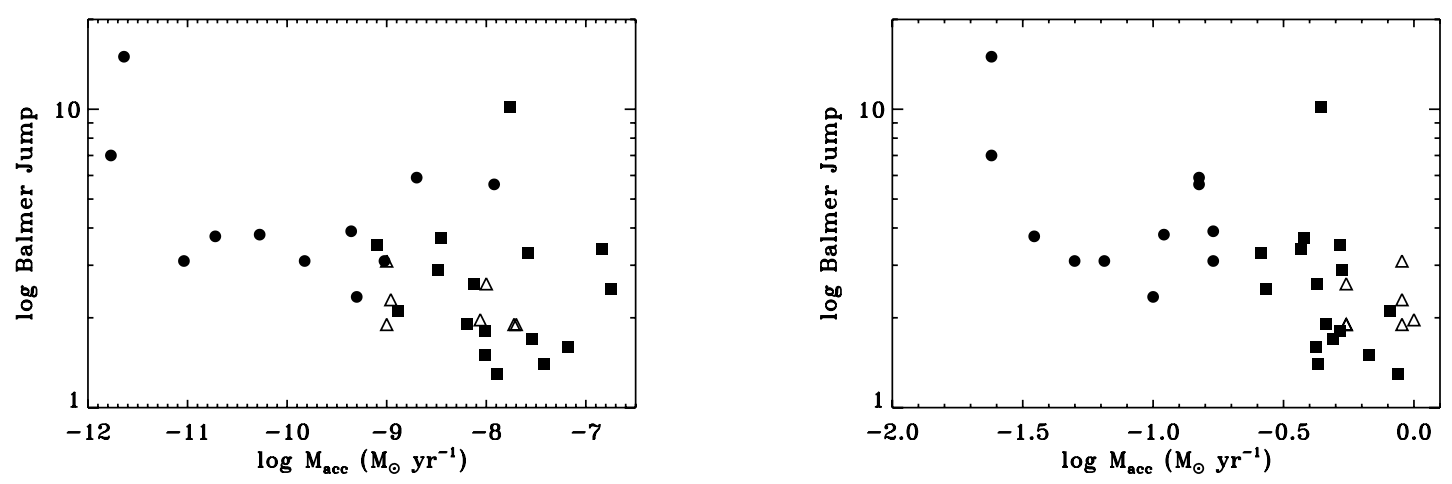

Fig. 13.- The Balmer jump vs. stellar mass (left) and $\dot{M}$ (right), as in Fig. 12. The squares are from Gullbring et al. (1998). The Balmer jump tends to increase to smaller $M$ and $\dot{M}$, suggesting that the accretion continuum is produced in gas with a lower electron density.

opacity in $\mathrm{H} \alpha$ to produce the broad line profiles. The percent of the stellar surface subtended by the accretion hot $\operatorname{spot}(\delta$; see Table 12$)^{7}$ is typically $0.01 \%-1 \%$ for low-mass stars and brown dwarfs, relative to $0.1 \%-10 \%$ for higher mass stars ( Valenti et al. 1993). These results should be fully accounted for as tests of the magnetospheric accretion models.

For regions with low extinction the UV excess method is the most accurate measure of accretion luminosity. The accretion luminosity can also be measured from the Paschen continuum at 3700-8000 A, albeit with larger uncertainties and reduced sensitivity to small accretion rates. UV excess measures of accretion can be applied to even lower mass objects than studied here, limited only by telescopic sensitivity. $\mathrm{H} \alpha$ line modeling may be a sufficient substitute for low-mass stars and brown dwarfs when UV excess measures are not possible, due either to large or uncertain extinction or distance or to instrumentation limitations. The $\mathrm{H} \alpha$ models at present cannot be applied to large $\dot{M}$ due to complex line profiles. They also cannot be applied to $\dot{M}<10^{-12} M_{\odot} \mathrm{yr}^{-1}$, below which the opacity in the $\mathrm{H} \alpha$ line wings may be insufficient to measure accretion (Muzerolle et al. 2003). However, $\mathrm{H} \alpha$ models may be better suited than UV excess measurements for small $\dot{M}$ onto higher mass/luminosity accretors, where the contrast between the Balmer continuum and photospheric emission is small. For example, the excess Balmer emission is difficult to detect onto the objects CIDA-14, V927 Tau, and $2 \mathrm{M} 0455+3028$, but the $10 \%$ width of the $\mathrm{H} \alpha$ line is large enough to suggest accretion. Additional comparisons of $\dot{M}$ between these two methods are required to better characterize the differences.

Secondary diagnostics, such as the $\mathrm{Ca}$ II IR triplet, $\mathrm{Pa} \beta$, or $\mathrm{Br} \gamma$ lines, are related to $L_{\text {acc }}$ or, less accurately, $\dot{M}$ by empirical relationships and can provide useful $\dot{M}$ estimates (see also Muzerolle et al. 1998; Natta et al. 2004; Mohanty et al. 2005). The many random uncertainties inherent in both the UV excess and $\mathrm{H} \alpha$ methods are reduced if the sample size is large enough. However, systematic uncertainties in both accretion measures are propagated into these correlations. Some uncertainty is also introduced into these relationships because the line emission may be produced in different gas than the accretion continuum and because wind absorption can reduce the line flux. Of the line luminosity-

\footnotetext{
7 The estimates of $\delta$ are inversely correlated with $L$, the length of the accretion slab, and $T$, the temperature of the accretion slab. The calculated $L$ may be nonphysical, and in this work they are larger here than the $\sim 10^{7}$ typical for Valenti et al. (1993). As a result, the difference in $\delta$ between the two works may be underestimated.
}

accretion luminosity relationships analyzed in Table 16 , the accretion luminosities estimated from the high Balmer lines and the He I 25016 line have the least amount of scatter. The line luminosity-accretion luminosity relationships are preferable to line flux $-\dot{M}$ relationships (Fig. 11), which have scatters of $\sim 0.75$ 1.0 dex. That this amount of scatter is similar to the scatter in the $M-\dot{M}$ relationship (see $\S 6.2$ ) suggests that the line flux $-\dot{M}$ relationships are not accurate.

Many of the commonly used lines (Ca II IR triplet, $\mathrm{Pa} \beta, \mathrm{Br} \gamma$ ) are less sensitive to $\dot{M} / M$ than either the $\mathrm{H} \alpha$ line profile or excess UV emission. They therefore provide a less complete picture of the full range of accretion. The completeness depends on mass if the detection limit of each diagnostic varies with spectral type. These diagnostics are often more accessible than modeling $\mathrm{H} \alpha$ profiles or measuring the UV excess and are particularly useful for variability studies of individual objects (e.g., Alencar et al. 2005; Azevedo et al. 2006) or in large samples (Natta et al. 2006).

\subsection{Accretion Rate versus Mass}

Figure 14 shows $\dot{M}$ versus $M$ for Taurus accretors with $A_{V}<$ 2.5 from Gullbring et al. (1998), Hartigan \& Kenyon (2003),

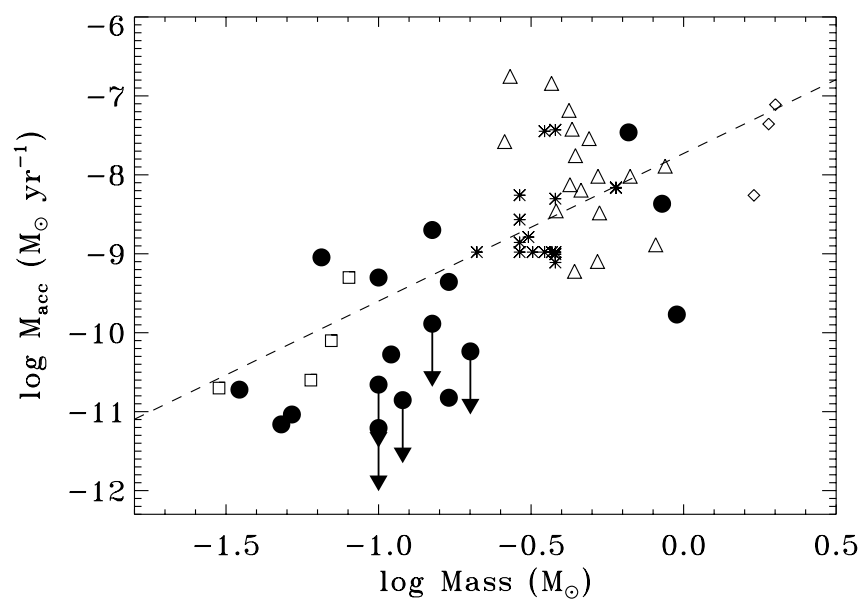

FIG. 14.-Mass vs. mass accretion rate for Taurus members with $A_{V}<2.5$ and measured accretion rates. The data plotted are $\dot{M}$ measured from excess UV continuum emission in this work (filled circles), from excess UV continuum emission measured by Gullbring et al. (1998; open triangles), optical veiling measured by Hartigan \& Kenyon (2003; asterisks), UV excess measured by Calvet et al. (2004; open diamonds), and $\mathrm{H} \alpha$ profile modeling (open squares; multiplied by 5 ; Muzerolle et al. 2005). The dashed line shows $\dot{M} \propto-7.7 M^{1.87}$. 

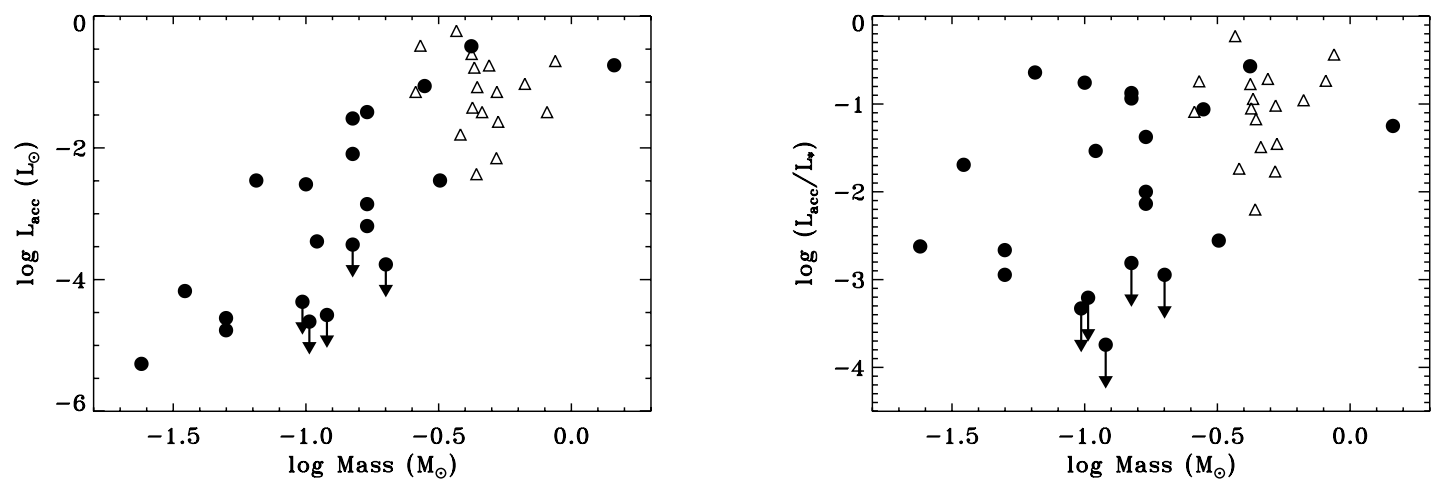

Fig. 15.- Relationship between mass and $L_{\mathrm{acc}}$ (left $)$ and $L_{\mathrm{acc}} / L_{\mathrm{bol}}$ (right), with circles from our data and open triangles from Gullbring et al. (1998). The sensitivity of measuring $L_{\text {acc }}$ and $L_{\text {acc }} / L_{\text {bol }}$ from the UV excess improves to lower masses.

Calvet et al. (2004), and Muzerolle et al. (2005) and from the low-resolution Keck I and HST spectra analyzed in this work. ${ }^{8}$ We use ASURV (Feigelson \& Nelson 1985) to calculate that

$$
\log \dot{M}=(1.87 \pm 0.26) \log M-(7.73 \pm 0.17),
$$

which is similar to previous estimates of $\dot{M} \propto M^{1.8}-M^{2.3}$ (Muzerolle et al. 2005; Mohanty et al. 2005; Natta et al. 2006). The standard deviation of the fit to $\dot{M}$ is 0.8 dex. This standard deviation is too large to be explained by intrinsic accretion variability or random methodological uncertainties, which suggests that stellar parameters other than mass also affect the accretion rate. A lack of completeness in our sampling of the true range in mass accretion rate for a given mass can bias the $\dot{M}-M^{\alpha}$ relationship by target selection and by sensitivity limits.

We specifically selected several targets (CIDA 1, GM Tau, and 2 M 0414+2811) based on our expectation of high $\dot{M}$. Most other stars were selected based in part on visual magnitude, which may tend to yield sources with higher $\dot{M}$. If this selection bias is only present in the current data, which focus on the low-mass range, then the $\alpha$ in the $\dot{M}-M^{\alpha}$ relationship will be underestimated. However, a similar bias could also be present in previous $\dot{M}$ measurements from samples of higher mass stars.

The $\dot{M}$ from higher mass accretors may be incomplete because sensitivity to $\dot{M} / M$ improves to smaller masses for both UV excess and $\mathrm{H} \alpha$ accretion measures. Figure 15 shows that accretion luminosity and the measured $L_{\mathrm{acc}} / L_{\mathrm{bol}}$ decrease with mass. The improved sensitivity to low $L_{\mathrm{acc}} / L_{\mathrm{bol}}$ at smaller masses results because the percentage of total photospheric emission that escapes at $<3700 \AA$ is much less for mid-M to late M dwarfs than for late K dwarfs. In Valenti et al. (1993) and Gullbring et al. (1998) the minimum value of $L_{\mathrm{acc}} / L_{\mathrm{bol}}$ is 0.008 and 0.018 , respectively, with most targets well above that limit. This detection limit leads to $\dot{M} \sim(5-10) \times 10^{-10} M_{\odot} \mathrm{yr}^{-1}$ for a $2 \mathrm{Myr}$ old, $0.7 M_{\odot}$ star with $L=0.57 L_{\odot}$. For the lower mass accretors studied here, accretion from $2 \mathrm{M} 0455+3028$ is at our detection limit with $L_{\text {acc }} / L_{\text {phot }} \sim 0.001$. For a $2 \mathrm{Myr}$ old, $0.1 M_{\odot}$ brown dwarf, the lowest detectable mass accretion rate is $\sim 10^{-11} M_{\odot} \mathrm{yr}^{-1}$. The $\dot{M} / M$ ratio is therefore about 5.5 times lower for a $0.1 M_{\odot}$ accretor than for a $0.7 M_{\odot}$ accretor. Incompleteness at higher masses would cause us to overestimate $\alpha$ in the $\dot{M}-M^{\alpha}$ relationship. On the other hand, inclusion of the five tentative accretors would increase $\alpha$ to 2.08 .

\footnotetext{
8 The Hartigan \& Kenyon (2003) data are used to recalculate accretion rates, as in $\S 5.1$. The Muzerolle et al. (2005) accretion rates are used only if they are not duplicates from this work and are multiplied by a factor of 5 (see $\S 5.3$ ). Duplicate measurements of $\dot{M}$ for the same star are averaged.
}

\section{CONCLUSIONS}

We analyzed blue spectra of 18 accreting very low mass stars and brown dwarfs obtained with LRIS on Keck I. Ours are the first data on the Balmer continuum and high Balmer line emission in this mass range. Most of this sample was selected based on the presence of accretion identified with other diagnostics. Our observations are compared with archival HST STIS spectra of the Balmer continuum from several higher mass stellar accretors. We obtain the following results:

1. We detect excess hydrogen Balmer continuum emission from 16 of the 18 mid-M to late M dwarfs in our sample. Eleven of these objects also show hydrogen Paschen continuum emission. This continuum is attributed to accretion for most of our objects. Weak Balmer continuum emission onto earlier type stars, including the K6 dwarf V836 Tau, can be attributed to accretion because nonaccretors show no evidence for chromospheric Balmer continuum emission. However, we cannot rule out a chromospheric origin for weak excess Balmer emission from mid-M dwarfs.

2. Measurements of the Balmer and Paschen continua emission yield $\dot{M}=10^{-12}$ to $10^{-8}$ for very low mass stars and brown dwarfs with masses from 0.024 to $0.17 M_{\odot}$. Most of these accretion rates are smaller than those typically measured for accreting $\sim 0.3-1.0 M_{\odot}$ stars. Analysis of the random and systematic errors indicates that these $\dot{M}$ may be underestimated by a factor of 1.2 (or 2.4 if the hydrogen continuum emission incident on the star is reprocessed into photospheric emission), with a relative uncertainty of $\sim 0.6$ dex. Comparing these $\dot{M}$ with $\dot{M}$ from other studies requires accounting for different assumptions regarding radiative transfer.

3. The Balmer jump tends to be larger and the Balmer continuum tends to be redder for stars with lower mass accretion rates. These properties indicate lower opacity (with $\log n_{e}=13.5$ for 2M 1207, compared with $\log n_{e} \sim 14.3$ for stars with higher $\dot{M}$ in the plane-parallel slab models) and lower temperature (with $T \sim 7000 \mathrm{~K}$ for $2 \mathrm{M} 1207$, compared with $\sim 9500$ for stars with higher $\dot{M})$.

4. The $\dot{M}$ calculated here are well correlated with, but $\sim 4$ 7 times larger than, the $\dot{M}$ calculated from published models of the $\mathrm{H} \alpha$ line profiles. We suggest that this offset is methodological, although the difference is within the range of the combined errors for both methods. The $\dot{M}$ measured here also correlates with $\mathrm{H} \alpha 10 \%$ width but introduces 0.9 dex uncertainty in $\dot{M}$ predicted from $\mathrm{H} \alpha 10 \%$ width alone.

5. We provide empirical relationships between $L_{\text {acc }}$ and $L_{\text {line }}$, which are often well correlated. In particular, estimating $L_{\text {acc }}$ 
from the luminosity in the high Balmer and many $\mathrm{He}$ I lines introduces an uncertainty of only $0.2-0.35$ dex. On the other hand, estimating the $L_{\text {acc }}$ from line surface fluxes alone introduces an uncertainty of $\sim 0.7-1.1 \mathrm{dex}$ in $\dot{M}$ estimates.

6. We find that $\log \dot{M} \propto M^{1.87}$ for Taurus stars with $A_{V}<2.5$, which is consistent with previous estimates. This relationship may be biased if the higher sensitivity of $\dot{M} / M$ at lower $M$ causes the percentage of accretors that are detected to also vary with $M$.

We thank Jeff Valenti and Chris Johns-Krull for use of their plane-parallel slab model codes that they developed in Valenti et al. (1993). We thank Russel White for discussion of the proposal and Nuria Calvet for discussion of the line emission. We thank the anonymous referee for helpful comments, which served to improve the structure and clarity of the paper.

Most of data presented herein were obtained at the W. M. Keck Observatory, which is operated as a scientific partnership among the California Institute of Technology, the University of California, and the National Aeronautics and Space Administration. The Observatory was made possible by the generous financial support of the W. M. Keck Foundation. Some of the data presented in this paper were obtained from the Multimission Archive at the Space Telescope Science Institute (MAST). STScI is operated by the Association of Universities for Research in Astronomy, Inc., under NASA contract NAS5-26555. Support for MAST for non-HST data is provided by the NASA Office of Space Science via grant NAG5-7584 and by other grants and contracts.

\section{APPENDIX A}

\section{PHOTOSPHERIC EMISSION}

The photospheric emission from M dwarfs is dominated by strong TiO and $\mathrm{CaH}$ bands at $\lambda>4800 \AA$ that can be used to estimate spectral type (e.g., Kirkpatrick et al. 1993). Table 3 lists spectral types for our targets estimated from the TiO $\lambda \lambda 7140$ and 8465 indices (Slesnick et al. 2006) and by visual comparison to existing Keck I LRIS spectra of M4.5-M9.5 M dwarfs. ${ }^{9}$ Most spectral types are consistent with existing literature estimates. We classify V927 Tau as an M5 star, compared with previous classifications of M3, M4.75, and M5.5.2M 0455+3028, S0439+2336, and MHO 6 have the same spectral type as V927 Tau and are classified as M5. MHO 7 is a slightly later spectral type than V927 Tau and is classified as M5.5, 0.25 classes later than in Briceño et al. (2002). The photospheric emission from CIDA 14 is nearly identical to MHO 7. S0518+2327 is about the same as MHO 7 and classified as M5.5, while KPNO Tau 11 is slightly later than MHO 7 and is classified as an M5.75. MHO 5 is between MHO 7 and CFHT 7 and is classified as M6. We confirm spectral types from Luhman (2004) for 2M 0438+2611, 2M 0439+2544, 2M 0441+2534, and 2M 0444+2512 and from Gizis (2002) for 2M 1207-3932. We conservatively assign a 0.5 subclass uncertainty to all spectral types.

The spectral types for GM Tau, CIDA 1, and 2M 0414+2811 are difficult to determine because of high veiling at $\lambda>7000 \AA$. In order to make some estimate, we assume that the accretion continuum emission is constant in flux per $\AA$ across short wavelength intervals. The depths of absorption bands are then compared to spectral type standards to estimate the spectral classification. The photospheric emission of CIDA 1 is consistent with M5, GM Tau with M5.5, and 2M 0414+2811 with CFHT 7. The spectral types of CIDA 1 and GM Tau are 0.5 and 1 class earlier, respectively, than that measured by White \& Basri (2003) and are both assigned an error of 1.0 subclasses.

\section{APPENDIX B}

\section{EXTINCTION}

Extinction estimates are important to convert the measured flux in the accretion continuum into a luminosity. Many of our targets were selected for observation based on low extinction. We adopt extinctions of $\sim 0.4,0.1,0.0$, and 0.0 for the nonaccretors V927 Tau, MHO 7, and CFHT 7 and the accretor 2M 0444+2512, respectively (Kenyon \& Hartmann 1995; Briceño et al. 2002; Guieu et al. 2007; Luhman 2004). We confirm these estimates with an accuracy of $\sim 1.0$ mag by comparing our flux-calibrated observations to existing Keck I LRIS red spectra (6000-10000 ̊) of photospheric templates.

The photospheric emission from the rest of our targets is then compared to those four templates to calculate an extinction. For accretors, we also add a flat accretion continuum at red wavelengths. We use the extinction law from Cardelli et al. (1989) and a ratio of total to selective extinction $\left(R_{V}\right)$ of 3.1. Some evidence suggests that $R_{V}>3.1$ in the Taurus molecular cloud because grains in the molecular cloud may be larger than those in the average interstellar medium (Whittet et al. 2001). If $R_{V}$ is large, the slope of extinction versus wavelength is shallow and $A_{V}$ will be underestimated. The spectrum of $2 \mathrm{M} 0438+2611$ is likely seen through an edge-on disk and suffers from gray extinction and scattering (Luhman et al. 2007) that is not corrected for here.

We confirm our extinction estimates for sources with strong veiling by assuming that the Paschen continuum between 4000 and $6000 \AA$ is flat (see $\S 3.1$ ), which is roughly consistent with observations of the higher mass accretors (e.g., Basri \& Batalha 1990; Hartigan et al. 1991), with observations of accretors with low $A_{V}$ (Gullbring et al. 2000), and with shock models (Calvet \& Gullbring 1998).

Our extinction estimates are listed in Table 3 . Any error in the $A_{V}$ for V927 Tau, MHO 7, CFHT 7, and 2M 0444+2512 will propagate to the remaining data. Our estimates are consistent with previous extinction estimates (Briceño et al. 2002; Luhman 2004), with a few exceptions. We estimate $A_{V} \sim 1.0$ and $\sim 0.7$ to $2 \mathrm{M} 0455+3028$ and $2 \mathrm{M} 0439+2544$, respectively, compared with $A_{J}=0.0$ and 0.07 from Luhman (2004). We measure $A_{V}=2.0 \pm 0.7$ to GM Tau, less than previous estimates of $\sim 4.2$ mag (Briceño et al. 2002 ; Guieu et al. 2007).

2M 1207-3932 is in the TW Hya Association and isolated from molecular gas. We assume $A_{V}=0$ to $2 \mathrm{M} 1207-3932$ and confirm that $A_{V}<1$ by comparison to a template LRIS M8 dwarf standard.

\footnotetext{
9 Obtained from http://www-int.stsci.edu/ inr/ultracool.html (Gizis et al. 2000a, 2000b; Kirkpatrick et al. 1999).
} 


\title{
APPENDIX C
}

\section{STELLAR RADIUS AND MASS}

Estimates for the stellar mass and radius are required to calculate mass accretion rates. The spectral class is converted to temperature using the scale derived by Luhman et al. (2003). We then use our spectral types, extinction estimates, synthetic $R$-band photometry (after correcting for the measured veiling), and Baraffe et al. (1998) evolutionary tracks to compute the stellar luminosity, mass, radius, and age (see Table 3). The calculated ages for the higher mass sample in Taurus are overestimated, which is consistent with known systematic problems in stellar evolutionary tracks (Hillenbrand et al. 2008).

A distance of $140 \mathrm{pc}$ is assumed for Taurus stars (Kenyon et al. 1994). Slesnick et al. (2006) identified S0518+2327 as an accretor away from the main Taurus molecular cloud and classify it as an intermediate-age star based on the depth of Na I gravity indices. The distance to $\mathrm{S} 0518+2327$ is therefore more uncertain than most other Taurus stars. We adopt a parallax distance of $54 \pm 3 \mathrm{pc}$ to 2M 1207-3932 (Gizis et al. 2007; see also Biller \& Close 2007) and of 56 pc for TW Hya (Wichmann et al. 1998).

Any binarity in our sample would reduce the stellar luminosity and estimated radius. Kraus et al. (2006) found that GM Tau and MHO 5 are spatially unresolved to $\sim 0.05^{\prime \prime}$ and are either single stars or spectroscopic binaries. We assume that our other targets are single stars with the exception of V927 Tau (see $\S \mathrm{D} 2$ ).

\section{APPENDIX D}

\section{NOTES ON INDIVIDUAL OBJECTS}

\author{
D1. 2MASS J1207-3932
}

2M 1207-3932 is an M8-L dwarf binary (Gizis 2002; Chauvin et al. 2004) in the 10 Myr old TW Hya Association (Webb et al. 1999; Mamajek 2005). The primary has an IR excess and ongoing accretion. The secondary is faint and not detectable in our data. We observed 2M 1207-3932 on both November 24 and February 8. The February observation was deeper and obtained with the target at lower air mass. The flux from the November integration is calibrated based on the strength of photospheric emission from 6000 to $8000 \AA$ measured in the February run. The blue and UV continuum emission was fainter in November than on February 8. Most of these parameters have a higher confidence for 2M 1207-3932 than the Taurus members.

Stelzer et al. (2007) applied the Natta et al. (2004) relationship for $\mathrm{H} \alpha 10 \%$ width to measure $\dot{M} \sim 10^{-10.1}$ to $10^{-9.8} M_{\odot} \mathrm{yr}^{-1}$ from 2M 1207-3932, which is 2 orders of magnitude larger than measured here. This difference is not likely attributed to variability because the $\mathrm{H} \alpha$ equivalent width during our February observation is the largest yet detected from 2M 1207-3932. During an epoch in 2003 with low $\dot{M}$ (Stelzer et al. 2007), both the equivalent width and $\mathrm{H} \alpha 10 \%$ width were small. The variable equivalent width in the $\mathrm{Ca}$ II 28542 line of $<0.3 \AA$ (Stelzer et al. 2007) corresponds to $\dot{M}<2.1 \times 10^{-12} M_{\odot} \mathrm{yr}^{-1}$ (Table 16), which confirms our low $\dot{M}$.

\section{D2. V927 Tau}

V927 Tau is a binary separated by $\sim 0.27^{\prime \prime}$ (Leinert et al. 1993; White \& Ghez 2001). Muzerolle et al. (2003) measured a broad 10\% width of $290 \mathrm{~km} \mathrm{~s}^{-1}$ and only a small self-reversal in the symmetric $\mathrm{H} \alpha$ line profile from V927 Tau. They suggested that this $\mathrm{H} \alpha$ emission may be produced by chromospheric flaring since most $\mathrm{H} \alpha$ profiles produced by accretion are asymmetric. At the time no excess IR or submillimeter emission had been detected from either component (Simon \& Prato 1995; White \& Ghez 2001; Andrews \& Williams 2005). However, McCabe et al. (2006) measured $K$-band magnitudes that were the same as measured by White \& Ghez (2001) but $L$-band emission that was brighter by 1 mag for both V927A and V927B. Such variability may suggest that the amount of warm dust in the disk is variable and that perhaps accretion onto V927 Tau may sometimes be present.

We detect some excess Balmer continuum and He I 25876 emission from V927 Tau relative to MHO 7. V927 Tau and its template MHO 7 are slightly different spectral types, which may lead to some discrepancy in this comparison. Such emission could be interpreted as either chromospheric or accretion related. Hartigan \& Kenyon (2003) found weak excess Paschen continuum emission from the secondary, if the secondary is classified as an M3.5 star. V927 Tau remains ambiguous as to whether accretion is ongoing.

\section{D3. 2MASS J0438+2611}

We obtain a short LRIS spectrum of 2M 0438+2611, which Luhman et al. (2007) describe as a young accreting star seen through an edge-on disk with a gray extinction of $5.5 \mathrm{mag}$. The gray extinction cannot be measured from the shape of the continuum emission, which is similar to that of $2 \mathrm{M} 0444+2512\left(A_{V}=0 \mathrm{mag}\right)$. If we assume that the two stars have the same radius, then we find $A_{V}=2.8 \mathrm{mag}$ to $2 \mathrm{M} 0438+2611$. That the optical forbidden lines are bright (see also Luhman 2004; Luhman et al. 2007) indicates that some of the outflow emission is at least slightly extended and not absorbed by the edge-on disk, consistent with other accretors seen through edge-on disks.

We classify $2 \mathrm{M} 0438+2611$ as a moderate accretor based on the detection of optical forbidden lines and nondetection of the Ca II IR triplet. We use the $\mathrm{H} \gamma$ line luminosity and Table 13 to calculate $L_{\text {acc }} \sim 4 \times 10^{-5} L_{\odot}$, or $\dot{M} \sim 1.5 \times 10^{-11} M_{\odot} \mathrm{yr}^{-1}$ (assuming that $M$ and $R$ are the same as for $2 \mathrm{M} 0444+2512)$. The upper limit for the Ca II 28542 equivalent width of $<1.2 \AA$ indicates $L_{\text {acc }}<1.6 \times 10^{-4} L_{\odot}$. We do not use the $\dot{M}$ of $2 \mathrm{M} 0438+2611$ in any analysis. 
D4. 2MASS J0414+2811

$2 \mathrm{M} 0414+2811$ is about a magnitude brighter and is bluer than $U B V$ photometry obtained by Grosso et al. (2007), which suggests a higher $\dot{M}$ during our observation.

\section{D5. MHO 5}

Emission in the [O I] $\lambda \lambda 6300,6363$ and $\mathrm{He}_{\mathrm{I}} \lambda 5876$ lines is detected with equivalent width typical of accretors. MHO 5 is therefore classified as an accretor, despite a low $\mathrm{H} \alpha 10 \%$ width of $154 \mathrm{~km} \mathrm{~s}^{-1}$ (Muzerolle et al. 2003). Ongoing accretion indicates that the weak excess Balmer continuum emission is likely produced by accretion, although we cannot rule out a chromospheric origin.

\section{D6. CIDA 14}

The excess Balmer continuum emission onto CIDA 14 is very weak and only detected because the photospheric emission is very similar to that from the nonaccretor MHO 7. We detect an equivalent width of $0.27 \AA$ in the [O I] line from CIDA 14 , which is slightly lower than the $0.4 \AA$ measured by Briceño et al. (1999) but larger than the $<0.1 \AA$ upper limit from high-resolution spectra by Muzerolle et al. (2003). This low upper limit may be underestimated if the line is broad. Muzerolle et al. (2003) measured an H $\alpha$ equivalent width of $289 \mathrm{~km} \mathrm{~s}^{-1}$. CIDA 14 is a likely accretor.

\section{D7. KPNO 11}

Several He I lines that are undetected from the nonaccretors are detected from KPNO 11 (Table 5). However, the strength of the He I $\lambda 5876$ line and the upper limit on the $\mathrm{Na}$ I D line luminosity are below the expected luminosity for the calculated $\dot{M}$. These luminosities may suggest that the excess Balmer continuum emission and He I lines are chromospheric. The H $\alpha 10 \%$ width of $182 \mathrm{~km} \mathrm{~s}^{-1}$ (Mohanty et al. 2005) is intermediate between accretors and nonaccretors.

\section{D8. GM Tau}

The flux ratio of Balmer line emission to Balmer continuum emission from GM Tau is much smaller than measured for any other source here, which requires a large optical depth in the slab.

\section{D9. S0518+2327}

S0518+2327 is an accretor located away from the main Taurus molecular cloud (Slesnick et al. 2006). The distance to this star is more uncertain than for the other Taurus stars studied here.

\section{D10. 2MASS J0436+2351}

The $U$-band atmospheric correction for $2 \mathrm{M} 0436+2351$ is somewhat uncertain. As a result, no slope for the Balmer continuum is listed in Table 5. The mass accretion rate for this star is also more uncertain than that of other stars in our sample.

\section{D11. DF Tau}

DF Tau is a $0.1^{\prime \prime}$ binary (Schaefer et al. 2006) that is unresolved in these spectra. When calculating the stellar radius, we assumed that the luminosities of the two stars are equal. STIS observed DF Tau three times with the G430L grating, two of which are analyzed here. The third observation shows much weaker photospheric and continuum emission with an accretion rate 10 times lower than the other observations (Herczeg et al. 2006). We do not analyze this third spectrum here because it was obtained with a narrower slit and could have included the secondary but not the primary.

\section{D12. DG Tau}

We measure $\dot{M}=4.1 \times 10^{-9} M_{\odot} \mathrm{yr}^{-1}$, an order of magnitude less than that measured by Gullbring et al. (2000). The spectrum of DG Tau during the Gullbring et al. (2000) observation was heavily veiled and an order of magnitude brighter than that detected here. The different $\dot{M}$ result from real variability. Strong emission is detected in forbidden lines and Fe I lines despite the lower $\dot{M}$.

\section{REFERENCES}

Alencar, S. H. P., Basri, G., Hartmann, L., \& Calvet, N. 2005, A\&A, 440, 595

Allred, J. C., Hawley, S. L., Abbett, W. P., \& Carlsson, M. 2006, ApJ, 644, 484

Andrews, S. M., \& Williams, J. P. 2005, ApJ, 631, 1134

Azevedo, R., Calvet, N., Hartmann, L., Folha, D. F. M., Gameiro, F., \& Muzerolle, J. 2006, A\&A, 456, 225

Baraffe, I., Chabrier, G., Allard, F., \& Hauschildt, P. H. 1998, A\&A, 337, 403

Basri, G., \& Batalha, C. 1990, ApJ, 363, 654

Batalha, C., Batalha, N. M., Alencar, S. H. P., Lopes, D. F., \& Duarte, E. S. 2002, ApJ, 580, 343
Batalha, C. C., Stout-Batalha, N. M., Basri, G., \& Terra, M. A. O. 1996, ApJS, 103,211

Beristain, G., Edwards, S., \& Kwan, J. 2001, ApJ, 551, 1037

Bertout, C., \& Genova, F. 2006, A\&A, 460, 499

Biller, B. A., \& Close, L. M. 2007, ApJ, 669, L41

Bouvier, J., et al. 2007, A\&A, 463, 1017

Briceño, C., Calvet, N., Gomez, M., Hartmann, L. W., Kenyon, S. J., \& Whitney, B. A. 1993, PASP, 105, 686

Briceño, C., Calvet, N., Kenyon, S., \& Hartmann, L. 1999, AJ, 118, 1354 
Briceño, C., Hartmann, L., Stauffer, J., \& Martin, E. 1998, AJ, 115, 2074

Briceño, C., Luhman, K. L., Hartmann, L., Stauffer, J. R., \& Kirkpatrick, J. D. 2002, ApJ, 580, 317

Calvet, N., \& Gullbring, E. 1998, ApJ, 509, 802

Calvet, N., Muzerolle, J., Briceño, C., Hernandez, J., Hartmann, L., Saucedo, J. L., \& Gordon, K. D. 2004, AJ, 128, 1294

Cardelli, J. A., Clayton, G. C., \& Mathis, J. S. 1989, ApJ, 345, 245

Cardini, D., \& Cassatella, A. 2007, ApJ, 666, 393

Chauvin, G., Lagrange, A.-M., Dumas, C., Zuckerman, B., Mouillet, D., Song, I., Beuzit, J.-L., \& Lowrance, P. 2004, A\&A, 425, L29

D'Antona, F., \& Mazzitelli, I. 1998, in ASP Conf. Ser. 134, Brown Dwarfs and Extrasolar Planets, ed. R. Rebolo, E. L. Martin, \& M. R. Zapatero Osorio (San Francisco: ASP), 442

Eason, E. L. E., Giampapa, M. S., Radick, R. R., Worden, S. P., \& Hege, E. K. 1992, AJ, 104, 1161

Edwards, S., Fischer, W., Hillenbrand, L. A., \& Kwan, J. 2006, ApJ, 646, 319

Feigelson, E. D., \& Kriss, G. A. 1981, ApJ, 248, L35

Feigelson, E. D., \& Nelson, P. I. 1985, ApJ, 293, 192

Giampapa, M. S., Worden, S. P., \& Linsky, J. L. 1982, ApJ, 258, 740

Gizis, J. E. 2002, ApJ, 575, 484

Gizis, J. E., Jao, W.-C., Subasavage, J. P., \& Henry, T. J. 2007, ApJ, 669, L45

Gizis, J. E., Monet, D. G., Reid, I. N., Kirkpatrick, J. D., \& Burgasser, A. J. 2000a, MNRAS, 311, 385

Gizis, J. E., Monet, D. G., Reid, I. N., Kirkpatrick, J. D., Liebert, J., \& Williams, R. 2000b, AJ, 120, 1085

Gizis, J. E., Reid, I. N., \& Hawley, S. L. 2002, AJ, 123, 3356

Grosso, N., et al. 2007, A\&A, 468, 391

Guieu, S., Dougados, C., Monin, J.-L., Magnier, E., \& Martin, E. L. 2006, A\&A, 446, 485

Guieu, S., et al. 2007, A\&A, 465, 855

Gullbring, E., Calvet, N., Muzerolle, J., \& Hartmann, L. 2000, ApJ, 544, 927

Gullbring, E., Hartmann, L., Briceño, C., \& Calvet, N. 1998, ApJ, 492, 323

Hamann, F. 1994, ApJS, 93, 485

Hamann, F., \& Persson, S. E. 1989, ApJ, 339, 1078 1992, ApJS, 82, 247

Hartigan, P., Edwards, S., \& Ghandour, L. 1995, ApJ, 452, 736

Hartigan, P., Edwards, S., \& Pierson, R. 2004, ApJ, 609, 261

Hartigan, P., \& Kenyon, S. J. 2003, ApJ, 583, 334

Hartigan, P., Kenyon, S. J., Hartmann, L., Strom, S. E., Edwards, S., Welty, A. D., \& Stauffer, J. 1991, ApJ, 382, 617

Hartmann, L., Hewett, R., \& Calvet, N. 1994, ApJ, 426, 669

Hawley, S. L., \& Pettersen, B. R. 1991, ApJ, 378, 725

Henize, K. G. 1954, ApJ, 119, 459

Herbig, G. H., \& Kameswara, R. N. 1972, ApJ, 174, 401

Herbig, G. H., \& Soderblom, D. R. 1980, ApJ, 242, 628

Herczeg, G. J., Linsky, J. L., Walter, F. M., Gahm, G. F., \& Johns-Krull, C. M. 2006, ApJS, 165, 256

Herczeg, G. J., Wood, B. E., Linsky, J. L., Valenti, J. A., \& Johns-Krull, C. M. 2004, ApJ, 607, 369

Herczeg, G. J., et al. 2005, AJ, 129, 2777

Hillenbrand, L. A., Bauermeister, A., \& White, R. J. 2008, in ASP Conf. Ser., Cool Stars 14, ed. G. van Belle (San Francisco: ASP), in press (astro-ph/0703642)

Jayawardhana, R., Mohanty, S., \& Basri, G. 2003, ApJ, 592, 282

Johns-Krull, C. M. 2007, ApJ, 664, 975

Johns-Krull, C. M., \& Basri, G. 1997, ApJ, 474, 433

Kastner, J. H., Huenemoerder, D. P., Schulz, N. S., Canizares, C. R., \& Weintraub, D. A. 2002, ApJ, 567, 434

Kenyon, S. J., Dobrzycka, D., \& Hartmann, L. W. 1994, AJ, 108, 1872
Kenyon, S. J., \& Hartmann, L. 1995, ApJS, 101, 117

Kirkpatrick, J. D., Kelly, D. M., Rieke, G. H., Liebert, J., Allard, F., \& Wehrse, R. 1993, ApJ, 402, 643

Kirkpatrick, J. D., et al. 1999, ApJ, 519, 802

Kraus, A. L., White, R. J., \& Hillenbrand, L. A. 2006, ApJ, 649, 306

Kurosawa, R., Harries, T. J., \& Symington, N. H. 2006, MNRAS, 370, 580

Leinert, C., Zinnecker, H., Weitzel, N., Christou, J., Ridgway, S. T., Jameson, R., Haas, M., \& Lenzen, R. 1993, A\&A, 278, 129

Luhman, K. L. 2004, ApJ, 617, 1216

Luhman, K. L., Stauffer, J. R., Muench, A. A., Rieke, G. H., Lada, E. A., Bouvier, J., \& Lada, C. J. 2003, ApJ, 593, 1093

Luhman, K. L., et al. 2006, ApJ, 647, 1180 2007, ApJ, 666, 1219

Mamajek, E. E. 2005, ApJ, 634, 1385

Massey, P., Strobel, K., Barnes, J. V., \& Anderson, E. 1988, ApJ, 328, 315

Mauas, P. J. D., \& Falchi, A. 1996, A\&A, 310, 245

McCabe, C., Ghez, A. M., Prato, L., Duchene, G., Fisher, R. S., \& Telesco, C. 2006, ApJ, 636, 932

McCarthy, J. K., et al. 1998, Proc. SPIE, 3355, 81

Mohanty, S., Jayawardhana, R., \& Basri, G. 2005, ApJ, 626, 498

Muzerolle, J., Calvet, N., Briceño, C., Hartmann, L., \& Hillenbrand, L. 2000, ApJ, 535, L47

Muzerolle, J., Calvet, N., \& Hartmann, L. 2001, ApJ, 550, 944

Muzerolle, J., Hartmann, L., \& Calvet, N. 1998, AJ, 116, 2965

Muzerolle, J., Hillenbrand, L., Calvet, N., Briceño, C., \& Hartmann, L. 2003, ApJ, 592, 266

Muzerolle, J., Luhman, K. L., Briceño, C., Hartmann, L., \& Calvet, N. 2005, ApJ, 625, 906

Natta, A., Testi, L., Muzerolle, J., Randich, S., Comeron, F., \& Persi, P. 2004, A\&A, 424, 603

Natta, A., Testi, L., \& Randich, S. 2006, A\&A, 452, 245

Oke, J. B. 1990, AJ, 99, 1621

Oke, J. B., Cohen, J. G., Carr, M., Cromer, J., Dingizian, A., \& Harris, F. H. 1995, PASP, 107, 375

Rauscher, E., \& Marcy, G. W. 2006, PASP, 118, 617

Reipurth, B., Pedrosa, A., \& Lago, M. T. V. T. 1996, A\&AS, 120, 229

Rucinski, S. M., \& Krautter, J. 1983, A\&A, 121, 217

Rydgren, A. E., \& Vrba, F. J. 1983, AJ, 88, 1017

Schaefer, G. H., Simon, M., Beck, T. L., Nelan, E., \& Prato, L. 2006, ApJ, 12, 2618

Scholz, A., \& Jayawardhana, R. 2006, ApJ, 638, 1056

Short, C. I., Doyle, J. G., \& Byrne, P. B. 1997, A\&A, 324, 196

Simon, M., \& Prato, L. 1995, ApJ, 450, 824

Slesnick, C. L., Carpenter, J. M., Hillenbrand, L. A., \& Mamajek, E. E. 2006, AJ, 132, 2665

Smak, J. 1964, ApJ, 139, 1095

Stelzer, B., Scholz, A., \& Jayawardhana, R. 2007, ApJ, 671, 842

Valenti, J. A., Basri, G., \& Johns, C. M. 1993, AJ, 106, 2024

Valenti, J. A., Fallon, A. A., \& Johns-Krull, C. M. 2003, ApJS, 147, 305

Webb, R. A., Zuckerman, B., Platais, I., Patience, J., White, R. J., Schwartz, M. J., \& McCarthy, C. 1999, ApJ, 512, L63

White, R. J., \& Basri, G. 2003, ApJ, 582, 1109

White, R. J., \& Ghez, A. M. 2001, ApJ, 556, 265

White, R. J., \& Hillenbrand, L. A. 2004, ApJ, 616, 998

Whittet, D. C. B., Gerakines, P. A., Hough, J. H., \& Shenoy, S. S. 2001, ApJ, 547,872

Wichmann, R., Bastian, U., Krautter, J., Jankovics, I., \& Ricinski, S. M. 1998, MNRAS, 301, L39 